\title{
A Microwave Spectroscopic and Quantum Chemical Study of 3-Butyne-1-selenol $\left(\mathrm{HSeCH}_{2} \mathrm{CH}_{2} \mathrm{C} \equiv \mathrm{CH}\right)$
}

\author{
Harald Møllendal ${ }^{\star} †$, Rajmund Mokso, ${ }^{\dagger}$ and Jean-Claude Guillemin ${ }^{\ddagger}$ \\ Centre for Theoretical and Computational Chemistry (CTCC), Department of Chemistry, \\ University of Oslo, P. O. Box 1033 Blindern, NO-0315 Oslo, Norway, and Sciences Chimique \\ de Rennes, UMR 6226 CNRS-ENSCR, École National Superieure de Chimie de Rennes, F. \\ 35700 Rennes, France
}

\section{Supporting Information}

tUniversity of Oslo

‡École National Superieure de Chimie de Rennes

*To whom correspondence should be addressed. Tel: +47 2285 5674; Fax: +47 2285 5441; Email: harald.mollendal@kjemi.uio.no 


\section{Comments for Spectroscopic Tables:}

Hamiltonian: Watson's A-reduction $I^{r}$ representation ${ }^{1}$

Transition labels: $J$ ' $K_{-1}$ '” $K_{+1}$ '” $J^{\prime} K_{-l}$ ' $J K_{+1}$ '

obs. - calc.: the observed - the calculated residuals

weight: the estimated uncertainties of the observed frequencies. Each transition is weighted according to the inverse square of its estimated uncertainty

t: Student's t-test ${ }^{2}$

distortion correction: centrifugal distortion correction

total: correction from quartic and sextic (if included) centrifugal distortion constants

higher: contribution from sextic centrifugal distortion constants

fixed: contribution from fixed quartic and/or fixed sextic centrifugal distortion constants. The values of fixed constants are also displayed.

rms deviation: root-mean-square deviation. Defined dimensionless in a weighted least-squares $\mathrm{fit}^{2}$

K: Ray's asymmetry parameter defined by $\kappa=(2 B-A-C) /(A-C)$

Inertial defect: defined by $\Delta=I_{c}-I_{a}-I_{b}$

Significant digits: the number of significant digits of each fitted constant are listed on the diagonal of the correlation matrix.

Units: the observed frequencies, the residuals, the distortion corrections, and the rotational constants are in $\mathrm{MHz}$, the quartic centrifugal distortion constants are in $\mathrm{kHz}$, the sextic centrifugal distortion constants are in $\mathrm{Hz}$, and the principal axis inertial constants and the inertial defect are in u $\AA^{2}$.

\section{References}

Vol. 6.

(1) Watson, J. K. G. Vibrational Spectra and Structure; Elsevier: Amsterdam, 1977;

(2) Hamilton, W. C. Statistics in Physical Science; The Ronald Press Company: New York, 1964. 
TABLE 1S: Microwave Spectrum of the Ground Vibrational state of Conformer II of $\mathrm{H}^{80} \mathrm{SeCH}_{2} \mathrm{CH}_{2} \mathrm{C} \equiv \mathrm{CH}$

Total number of transitions used in the least-squares fit: 287

\begin{tabular}{|c|c|c|c|c|c|c|c|c|c|c|c|c|}
\hline & & $\operatorname{tans}$ & siti & ion & & . frequency & $\begin{array}{l}\text { obs.- } \\
\text { calc. }\end{array}$ & weight & t & $\begin{array}{r}\text { distort } \\
\text { total }\end{array}$ & $\begin{array}{l}\text { corre } \\
\text { higher }\end{array}$ & $\begin{array}{l}=\text { ions } \\
\text { fixed }\end{array}$ \\
\hline 22 & 7 & 16 & 23 & 7 & 17 & 45430.680 & 0.081 & 0.10 & 0.6 & 4.351 & 0.085 & $2.5 E-4$ \\
\hline 22 & 7 & 15 & 23 & 7 & 16 & 45430.680 & 0.081 & 0.10 & 0.6 & 4.351 & 0.085 & $2.5 E-4$ \\
\hline 22 & 8 & 15 & 23 & 8 & 16 & 45432.810 & 0.029 & 0.10 & 0.2 & 6.911 & 0.111 & 3. $4 E-4$ \\
\hline 22 & 8 & 14 & 23 & 8 & 15 & 45432.810 & 0.029 & 0.10 & 0.2 & 6.911 & 0.111 & 3. $4 E-4$ \\
\hline 22 & 9 & 14 & 23 & 9 & 15 & 45435.390 & -0.035 & 0.10 & -0.3 & 9.812 & 0.141 & 3. $3 E-4$ \\
\hline 22 & 9 & 13 & 23 & 9 & 14 & 45435.390 & -0.035 & 0.10 & -0.3 & 9.812 & 0.141 & 3. $3 E-4$ \\
\hline 22 & 10 & 13 & 23 & 10 & 14 & 45438.610 & 0.125 & 0.10 & 0.9 & 13.054 & 0.174 & 2. $4 E-4$ \\
\hline 22 & 10 & 12 & 23 & 10 & 13 & 45438.610 & 0.125 & 0.10 & 0.9 & 13.054 & 0.174 & 2. $4 E-4$ \\
\hline 22 & 11 & 12 & 23 & 11 & 13 & 45441.820 & -0.114 & 0.10 & -0.9 & 16.638 & 0.211 & $9.1 E-5$ \\
\hline 22 & 11 & 11 & 23 & 11 & 12 & 45441.820 & -0.114 & 0.10 & -0.9 & 16.638 & 0.211 & $9.1 E-5$ \\
\hline 22 & 12 & 11 & 23 & 12 & 12 & 45445.680 & -0.077 & 0.10 & -0.6 & 20.562 & 0.251 & $-9.9 E-5$ \\
\hline 22 & 12 & 10 & 23 & 12 & 11 & 45445.680 & -0.077 & 0.10 & -0.6 & 20.562 & 0.251 & $-9.9 E-5$ \\
\hline 22 & 13 & 10 & 23 & 13 & 11 & 45449.680 & -0.264 & 0.10 & -2.0 & 24.828 & 0.294 & $-3 \cdot 3 E-4$ \\
\hline 22 & 13 & 9 & 23 & 13 & 10 & 45449.680 & -0.264 & 0.10 & -2.0 & 24.828 & 0.294 & $-3 \cdot 3 E-4$ \\
\hline 22 & 14 & 9 & 23 & 14 & 10 & 45454.400 & -0.088 & 0.10 & -0.7 & 29.436 & 0.341 & $-5.9 E-4$ \\
\hline 22 & 14 & 8 & 23 & 14 & 9 & 45454.400 & -0.088 & 0.10 & -0.7 & 29.436 & 0.341 & $-5.9 E-4$ \\
\hline 23 & 9 & 15 & 24 & 9 & 16 & 47410.670 & 0.062 & 0.10 & 0.5 & 9.880 & 0.160 & 3. $9 E-4$ \\
\hline 23 & 9 & 14 & 24 & 9 & 15 & 47410.670 & 0.062 & 0.10 & 0.5 & 9.880 & 0.160 & 3. $9 \mathrm{E}-4$ \\
\hline 23 & 10 & 14 & 24 & 10 & 15 & 47413.730 & -0.057 & 0.10 & -0.4 & 13.266 & 0.198 & 3. $2 E-4$ \\
\hline 23 & 10 & 13 & 24 & 10 & 14 & 47413.730 & -0.057 & 0.10 & -0.4 & 13.266 & 0.198 & 3. $2 E-4$ \\
\hline 23 & 11 & 13 & 24 & 11 & 14 & 47417.160 & -0.217 & 0.10 & -1.6 & 17.009 & 0.239 & 1. $9 E-4$ \\
\hline 23 & 11 & 12 & 24 & 11 & 13 & 47417.160 & -0.217 & 0.10 & -1.6 & 17.009 & 0.239 & 1. $9 E-4$ \\
\hline 23 & 12 & 12 & 24 & 12 & 13 & 47421.230 & -0.130 & 0.15 & -0.6 & 21.108 & 0.285 & $3.6 E-6$ \\
\hline 23 & 12 & 11 & 24 & 12 & 12 & 47421.230 & -0.130 & 0.15 & -0.6 & 21.108 & 0.285 & 3. $6 \mathrm{E}-6$ \\
\hline 24 & 7 & 18 & 25 & 7 & 19 & 49380.730 & 0.106 & 0.10 & 0.8 & 3.954 & 0.110 & 2. $0 E-4$ \\
\hline 24 & 7 & 17 & 25 & 7 & 18 & 49380.730 & 0.106 & 0.10 & 0.8 & 3.954 & 0.110 & 2. $0 E-4$ \\
\hline 24 & 8 & 17 & 25 & 8 & 18 & 49382.860 & -0.067 & 0.10 & -0.5 & 6.742 & 0.143 & 4. $0 \mathrm{E}-4$ \\
\hline 24 & 8 & 16 & 25 & 8 & 17 & 49382.860 & -0.067 & 0.10 & -0.5 & 6.742 & 0.143 & 4. $0 E-4$ \\
\hline 24 & 9 & 16 & 25 & 9 & 17 & 49385.870 & 0.114 & 0.10 & 0.9 & 9.902 & 0.181 & $4.5 E-4$ \\
\hline 24 & 9 & 15 & 25 & 9 & 16 & 49385.870 & 0.114 & 0.10 & 0.9 & 9.902 & 0.181 & $4.5 E-4$ \\
\hline 24 & 10 & 15 & 25 & 10 & 16 & 49389.070 & 0.017 & 0.10 & 0.1 & 13.432 & 0.224 & $4.1 E-4$ \\
\hline 24 & 10 & 14 & 25 & 10 & 15 & 49389.070 & 0.017 & 0.10 & 0.1 & 13.432 & 0.224 & $4.1 E-4$ \\
\hline 24 & 14 & 11 & 25 & 14 & 12 & 49406.390 & -0.016 & 0.10 & -0.1 & 31.272 & 0.438 & $-3.8 E-4$ \\
\hline 24 & 14 & 10 & 25 & 14 & 11 & 49406.390 & -0.016 & 0.10 & -0.1 & 31.272 & 0.438 & $-3.8 E-4$ \\
\hline 25 & 8 & 18 & 26 & 8 & 19 & 51358.140 & 0.190 & 0.10 & 1.4 & 6.587 & 0.161 & $4.1 E-4$ \\
\hline 25 & 8 & 17 & 26 & 8 & 18 & 51358.140 & 0.190 & 0.10 & 1.4 & 6.587 & 0.161 & $4.1 \mathrm{E}-4$ \\
\hline 25 & 9 & 17 & 26 & 9 & 18 & 51361.050 & 0.182 & 0.15 & 0.9 & 9.876 & 0.204 & $5.1 E-4$ \\
\hline 25 & 9 & 16 & 26 & 9 & 17 & 51361.050 & 0.182 & 0.15 & 0.9 & 9.876 & 0.204 & $5.1 E-4$ \\
\hline 25 & 10 & 16 & 26 & 10 & 17 & 51364.450 & 0.170 & 0.10 & 1.3 & 13.552 & 0.252 & 4. $9 E-4$ \\
\hline 25 & 10 & 15 & 26 & 10 & 16 & 51364.450 & 0.170 & 0.10 & 1.3 & 13.552 & 0.252 & 4. $9 E-4$ \\
\hline 25 & 11 & 15 & 26 & 11 & 16 & 51368.230 & 0.081 & 0.10 & 0.6 & 17.614 & 0.304 & 3. $9 E-4$ \\
\hline 25 & 11 & 14 & 26 & 11 & 15 & 51368.230 & 0.081 & 0.10 & 0.6 & 17.614 & 0.304 & 3. $9 E-4$ \\
\hline 25 & 12 & 14 & 26 & 12 & 15 & 51372.430 & -0.021 & 0.10 & -0.2 & 22.063 & 0.362 & 2. $3 E-4$ \\
\hline 25 & 12 & 13 & 26 & 12 & 14 & 51372.430 & -0.021 & 0.10 & -0.2 & 22.063 & 0.362 & 2. $3 E-4$ \\
\hline 26 & 7 & 20 & 27 & 7 & 21 & 53330.550 & 0.017 & 0.10 & 0.1 & 3.364 & 0.138 & 2. $9 E-5$ \\
\hline 26 & 7 & 19 & 27 & 7 & 20 & 53330.550 & 0.017 & 0.10 & 0.1 & 3.364 & 0.138 & 2. $9 E-5$ \\
\hline 26 & 8 & 19 & 27 & 8 & 20 & 53333.140 & 0.200 & 0.10 & 1.5 & 6.381 & 0.180 & 4. $0 E-4$ \\
\hline 26 & 8 & 18 & 27 & 8 & 19 & 53333.140 & 0.200 & 0.10 & 1.5 & 6.381 & 0.180 & 4. $0 E-4$ \\
\hline 26 & 10 & 17 & 27 & 10 & 18 & 53339.440 & -0.029 & 0.10 & -0.2 & 13.621 & 0.282 & $5.8 E-4$ \\
\hline & 10 & 16 & 27 & 10 & 17 & 53339.440 & -0.029 & 0.10 & -0.2 & 13.621 & 0.282 & $5.8 E-4$ \\
\hline
\end{tabular}




\begin{tabular}{|c|c|c|c|c|c|c|c|c|c|c|c|c|}
\hline 26 & 11 & 16 & 27 & 11 & 17 & 53343.540 & 0.066 & 0.10 & 0.5 & 17.845 & 0.341 & 5. $0 E-4$ \\
\hline 26 & 11 & 15 & 27 & 11 & 16 & 53343.540 & 0.066 & 0.10 & 0.5 & 17.845 & 0.341 & $5.0 E-4$ \\
\hline 26 & 12 & 15 & 27 & 12 & 16 & 53347.920 & -0.014 & 0.10 & -0.1 & 22.470 & 0.406 & $3.5 E-4$ \\
\hline 26 & 12 & 14 & 27 & 12 & 15 & 53347.920 & -0.014 & 0.10 & -0.1 & 22.470 & 0.406 & $3.5 E-4$ \\
\hline 26 & 14 & 13 & 27 & 14 & 14 & 53358.300 & 0.138 & 0.10 & 1.0 & 32.927 & 0.552 & $-1 \cdot 2 E-4$ \\
\hline 26 & 14 & 12 & 27 & 14 & 13 & 53358.300 & 0.138 & 0.10 & 1.0 & 32.927 & 0.552 & $-1 \cdot 2 E-4$ \\
\hline 27 & 7 & 21 & 28 & 7 & 22 & 55305.620 & 0.178 & 0.10 & 1.3 & 2.992 & 0.154 & $-1 \cdot 1 E-4$ \\
\hline 27 & 7 & 20 & 28 & 7 & 21 & 55305.620 & 0.178 & 0.10 & 1.3 & 2.992 & 0.154 & $-1 \cdot 1 E-4$ \\
\hline 27 & 8 & 20 & 28 & 8 & 21 & 55307.930 & 0.037 & 0.10 & 0.3 & 6.124 & 0.201 & $3.6 \mathrm{E}-4$ \\
\hline 27 & 8 & 19 & 28 & 8 & 20 & 55307.930 & 0.037 & 0.10 & 0.3 & 6.124 & 0.201 & 3. $6 E-4$ \\
\hline 27 & 9 & 19 & 28 & 9 & 20 & 55310.980 & 0.001 & 0.10 & 0.0 & 9.674 & 0.255 & $5.9 \mathrm{E}-4$ \\
\hline 27 & 9 & 18 & 28 & 9 & 19 & 55310.980 & 0.001 & 0.10 & 0.0 & 9.674 & 0.255 & 5. $9 E-4$ \\
\hline 27 & 10 & 18 & 28 & 10 & 19 & 55314.570 & -0.046 & 0.10 & -0.3 & 13.640 & 0.314 & $6.5 E-4$ \\
\hline 27 & 10 & 17 & 28 & 10 & 18 & 55314.570 & -0.046 & 0.10 & -0.3 & 13.640 & 0.314 & $6.5 E-4$ \\
\hline 27 & 13 & 15 & 28 & 13 & 16 & 55328.340 & -0.112 & 0.10 & -0.8 & 28.045 & 0.531 & $2.7 E-4$ \\
\hline 27 & 13 & 14 & 28 & 13 & 15 & 55328.340 & -0.112 & 0.10 & -0.8 & 28.045 & 0.531 & $2.7 E-4$ \\
\hline 27 & 14 & 14 & 28 & 14 & 15 & 55334.040 & 0.065 & 0.10 & 0.5 & 33.682 & 0.616 & 1. $7 E-5$ \\
\hline 27 & 14 & 13 & 28 & 14 & 14 & 55334.040 & 0.065 & 0.10 & 0.5 & 33.682 & 0.616 & 1. $7 E-5$ \\
\hline 27 & 15 & 13 & 28 & 15 & 14 & 55339.920 & -0.018 & 0.10 & -0.1 & 39.736 & 0.707 & $-2 \cdot 9 E-4$ \\
\hline 27 & 15 & 12 & 28 & 15 & 13 & 55339.920 & -0.018 & 0.10 & -0.1 & 39.736 & 0.707 & $-2 \cdot 9 E-4$ \\
\hline 28 & 8 & 21 & 29 & 8 & 22 & 57282.850 & 0.041 & 0.10 & 0.3 & 5.813 & 0.223 & 3. $0 E-4$ \\
\hline 28 & 8 & 20 & 29 & 8 & 21 & 57282.850 & 0.041 & 0.10 & 0.3 & 5.813 & 0.223 & 3. $0 \mathrm{E}-4$ \\
\hline 28 & 9 & 20 & 29 & 9 & 21 & 57286.090 & 0.115 & 0.10 & 0.9 & 9.493 & 0.283 & $6.0 \mathrm{E}-4$ \\
\hline 28 & 9 & 19 & 29 & 9 & 20 & 57286.090 & 0.115 & 0.10 & 0.9 & 9.493 & 0.283 & $6.0 E-4$ \\
\hline 28 & 10 & 19 & 29 & 10 & 20 & 57289.800 & 0.079 & 0.10 & 0.6 & 13.606 & 0.349 & 7. $2 E-4$ \\
\hline 28 & 10 & 18 & 29 & 10 & 19 & 57289.800 & 0.079 & 0.10 & 0.6 & 13.606 & 0.349 & 7. $2 E-4$ \\
\hline 28 & 11 & 18 & 29 & 11 & 19 & 57293.930 & -0.068 & 0.10 & -0.5 & 18.152 & 0.422 & 7. $1 \mathrm{E}-4$ \\
\hline 28 & 11 & 17 & 29 & 11 & 18 & 57293.930 & -0.068 & 0.10 & -0.5 & 18.152 & 0.422 & $7.1 \mathrm{E}-4$ \\
\hline 28 & 12 & 17 & 29 & 12 & 18 & 57298.690 & -0.082 & 0.10 & -0.6 & 23.131 & 0.503 & $6.0 E-4$ \\
\hline 28 & 12 & 16 & 29 & 12 & 17 & 57298.690 & -0.082 & 0.10 & -0.6 & 23.131 & 0.503 & $6.0 E-4$ \\
\hline 28 & 13 & 16 & 29 & 13 & 17 & 57304.140 & 0.116 & 0.20 & 0.4 & 28.542 & 0.590 & 4. $2 E-4$ \\
\hline 28 & 13 & 15 & 29 & 13 & 16 & 57304.140 & 0.116 & 0.20 & 0.4 & 28.542 & 0.590 & 4. $2 E-4$ \\
\hline 28 & 14 & 15 & 29 & 14 & 16 & 57309.430 & -0.313 & 0.15 & -1.6 & 34.386 & 0.684 & 1. $7 E-4$ \\
\hline 28 & 14 & 14 & 29 & 14 & 15 & 57309.430 & -0.313 & 0.15 & $-1 \cdot 6$ & 34.386 & 0.684 & 1. $7 E-4$ \\
\hline 28 & 15 & 14 & 29 & 15 & 15 & 57316.090 & 0.171 & 0.10 & 1.3 & 40.664 & 0.785 & $-1.4 E-4$ \\
\hline 28 & 15 & 13 & 29 & 15 & 14 & 57316.090 & 0.171 & 0.10 & 1.3 & 40.664 & 0.785 & $-1 \cdot 4 E-4$ \\
\hline 29 & 7 & 23 & 30 & 7 & 24 & 59254.890 & -0.271 & 0.10 & -2.0 & 2.082 & 0.189 & $-5 \cdot 3 E-4$ \\
\hline 29 & 7 & 23 & 30 & 7 & 24 & 59254.890 & -0.271 & 0.10 & -2.0 & 2.082 & 0.189 & $-5 \cdot 3 E-4$ \\
\hline 29 & 8 & 22 & 30 & 8 & 23 & 59257.750 & 0.064 & 0.15 & 0.3 & 5.446 & 0.247 & 2. $0 E-4$ \\
\hline 29 & 8 & 21 & 30 & 8 & 22 & 59257.750 & 0.064 & 0.15 & 0.3 & 5.446 & 0.247 & 2. $0 E-4$ \\
\hline 29 & 9 & 21 & 30 & 9 & 22 & 59261.040 & 0.112 & 0.10 & 0.8 & 9.257 & 0.313 & 6. $0 E-4$ \\
\hline 29 & 9 & 20 & 30 & 9 & 21 & 59261.040 & 0.112 & 0.10 & 0.8 & 9.257 & 0.313 & $6.0 \mathrm{E}-4$ \\
\hline 29 & 10 & 20 & 30 & 10 & 21 & 59264.740 & -0.043 & 0.10 & -0.3 & 13.517 & 0.386 & $7.8 E-4$ \\
\hline 29 & 10 & 19 & 30 & 10 & 20 & 59264.740 & -0.043 & 0.10 & -0.3 & 13.517 & 0.386 & 7. $8 E-4$ \\
\hline 30 & 9 & 22 & 31 & 9 & 23 & 61235.970 & 0.131 & 0.10 & 1.0 & 8.964 & 0.345 & $5.7 E-4$ \\
\hline 30 & 9 & 21 & 31 & 9 & 22 & 61235.970 & 0.131 & 0.10 & 1.0 & 8.964 & 0.345 & $5.7 E-4$ \\
\hline 30 & 10 & 21 & 31 & 10 & 22 & 61239.770 & -0.029 & 0.10 & -0.2 & 13.371 & 0.426 & $8 \cdot 2 \mathrm{E}-4$ \\
\hline 30 & 10 & 20 & 31 & 10 & 21 & 61239.770 & -0.029 & 0.10 & -0.2 & 13.371 & 0.426 & 8. $2 E-4$ \\
\hline 30 & 11 & 20 & 31 & 11 & 21 & 61244.210 & -0.130 & 0.10 & -1.0 & 18.242 & 0.516 & $9.0 E-4$ \\
\hline 30 & 11 & 19 & 31 & 11 & 20 & 61244.210 & -0.130 & 0.10 & $-1 \cdot 0$ & 18.242 & 0.516 & $9.0 E-4$ \\
\hline 30 & 12 & 19 & 31 & 12 & 20 & 61249.300 & -0.124 & 0.10 & -0.9 & 23.576 & 0.614 & $8.6 E-4$ \\
\hline 30 & 12 & 18 & 31 & 12 & 19 & 61249.300 & -0.124 & 0.10 & -0.9 & 23.576 & 0.614 & $8.6 E-4$ \\
\hline 30 & 13 & 18 & 31 & 13 & 19 & 61254.910 & -0.118 & 0.10 & -0.9 & 29.374 & 0.721 & 7. $1 \mathrm{E}-4$ \\
\hline 30 & 13 & 17 & 31 & 13 & 18 & 61254.910 & -0.118 & 0.10 & -0.9 & 29.374 & 0.721 & 7. $1 E-4$ \\
\hline 30 & 14 & 17 & 31 & 14 & 18 & 61260.940 & -0.197 & 0.10 & -1.5 & 35.636 & 0.836 & 4. $9 E-4$ \\
\hline 30 & 14 & 16 & 31 & 14 & 17 & 61260.940 & -0.197 & 0.10 & -1.5 & 35.636 & 0.836 & $4.9 \mathrm{E}-4$ \\
\hline 30 & 16 & 15 & 31 & 16 & 16 & 61274.820 & -0.007 & 0.10 & -0.0 & 49.551 & 1.092 & $-1 \cdot 5 E-4$ \\
\hline 30 & 16 & 14 & 31 & 16 & 15 & 61274.820 & -0.007 & 0.10 & -0.0 & 49.551 & 1.092 & $-1 \cdot 5 E-4$ \\
\hline 31 & 8 & 24 & 32 & 8 & 25 & 63207.480 & 0.159 & 0.10 & 1.2 & 4.536 & 0.300 & $-1 \cdot 1 E-4$ \\
\hline 31 & 8 & 23 & 32 & 8 & 24 & 63207.480 & 0.159 & 0.10 & 1.2 & 4.536 & 0.300 & $-1 \cdot 1 E-4$ \\
\hline 31 & 9 & 23 & 32 & 9 & 24 & 63210.770 & 0.065 & 0.10 & 0.5 & 8.612 & 0.380 & $5.1 E-4$ \\
\hline 31 & 9 & 22 & 32 & 9 & 23 & 63210.770 & 0.065 & 0.10 & 0.5 & 8.612 & 0.380 & $5.1 E-4$ \\
\hline
\end{tabular}




\begin{tabular}{|c|c|c|c|c|c|c|c|c|c|c|c|c|}
\hline 31 & 10 & 22 & 32 & 10 & 23 & 63214.840 & 0.072 & 0.10 & 0.5 & 13.167 & 0.469 & $8.5 E-4$ \\
\hline 31 & 10 & 21 & 32 & 10 & 22 & 63214.840 & 0.072 & 0.10 & 0.5 & 13.167 & 0.469 & $8.5 E-4$ \\
\hline 31 & 11 & 21 & 32 & 11 & 22 & 63219.480 & 0.041 & 0.10 & 0.3 & 18.201 & 0.568 & $9.8 E-4$ \\
\hline 31 & 11 & 20 & 32 & 11 & 21 & 63219.480 & 0.041 & 0.10 & 0.3 & 18.201 & 0.568 & $9.8 E-4$ \\
\hline 31 & 12 & 20 & 32 & 12 & 21 & 63224.540 & -0.137 & 0.10 & $-1 \cdot 0$ & 23.714 & 0.675 & $9.8 E-4$ \\
\hline 31 & 12 & 19 & 32 & 12 & 20 & 63224.540 & -0.137 & 0.10 & -1.0 & 23.714 & 0.675 & $9.8 E-4$ \\
\hline 31 & 13 & 19 & 32 & 13 & 20 & 63230.220 & -0.236 & 0.10 & -1.8 & 29.706 & 0.793 & 8. $6 E-4$ \\
\hline 31 & 13 & 18 & 32 & 13 & 19 & 63230.220 & -0.236 & 0.10 & -1.8 & 29.706 & 0.793 & 8. $6 E-4$ \\
\hline 31 & 14 & 18 & 32 & 14 & 19 & 63236.450 & -0.309 & 0.10 & $-2 \cdot 3$ & 36.178 & 0.919 & $6.6 \mathrm{E}-4$ \\
\hline 31 & 14 & 17 & 32 & 14 & 18 & 63236.450 & -0.309 & 0.10 & -2.3 & 36.178 & 0.919 & 6. $6 E-4$ \\
\hline 31 & 15 & 17 & 32 & 15 & 18 & 63243.470 & -0.104 & 0.10 & -0.8 & 43.128 & 1.055 & $3.8 E-4$ \\
\hline 31 & 15 & 16 & 32 & 15 & 17 & 63243.470 & -0.104 & 0.10 & -0.8 & 43.128 & 1.055 & $3.8 E-4$ \\
\hline 31 & 16 & 16 & 32 & 16 & 17 & 63250.920 & 0.027 & 0.10 & 0.2 & 50.559 & 1.201 & $3.7 E-5$ \\
\hline 31 & 16 & 15 & 32 & 16 & 16 & 63250.920 & 0.027 & 0.10 & 0.2 & 50.559 & 1.201 & $3.7 E-5$ \\
\hline 31 & 17 & 15 & 32 & 17 & 16 & 63258.730 & 0.020 & 0.10 & 0.2 & 58.468 & 1.355 & $-3.6 E-4$ \\
\hline 31 & 17 & 14 & 32 & 17 & 15 & 63258.730 & 0.020 & 0.10 & 0.2 & 58.468 & 1.355 & $-3.6 E-4$ \\
\hline 31 & 19 & 13 & 32 & 19 & 14 & 63275.920 & 0.095 & 0.10 & 0.7 & 75.725 & 1.693 & $-1 \cdot 3 E-3$ \\
\hline 31 & 19 & 12 & 32 & 19 & 13 & 63275.920 & 0.095 & 0.10 & 0.7 & 75.725 & 1.693 & $-1 \cdot 3 E-3$ \\
\hline 32 & 7 & 26 & 33 & 7 & 27 & 65179.500 & 0.024 & 0.10 & 0.2 & 0.276 & 0.252 & $-1 \cdot 6 E-3$ \\
\hline 32 & 7 & 25 & 33 & 7 & 26 & 65179.500 & 0.024 & 0.10 & 0.2 & 0.276 & 0.252 & $-1 \cdot 6 E-3$ \\
\hline 32 & 8 & 25 & 33 & 8 & 26 & 65182.300 & 0.226 & 0.10 & 1.7 & 3.990 & 0.329 & $-3.4 E-4$ \\
\hline 32 & 8 & 24 & 33 & 8 & 25 & 65182.300 & 0.226 & 0.10 & 1.7 & 3.990 & 0.329 & $-3.4 E-4$ \\
\hline 32 & 9 & 24 & 33 & 9 & 25 & 65185.710 & 0.186 & 0.10 & 1.4 & 8.199 & 0.417 & 4. $2 E-4$ \\
\hline 32 & 9 & 23 & 33 & 9 & 24 & 65185.710 & 0.186 & 0.10 & 1.4 & 8.199 & 0.417 & $4 \cdot 2 E-4$ \\
\hline 32 & 10 & 23 & 33 & 10 & 24 & 65189.620 & -0.068 & 0.10 & -0.5 & 12.902 & 0.514 & $8.5 E-4$ \\
\hline 32 & 10 & 22 & 33 & 10 & 23 & 65189.620 & -0.068 & 0.10 & -0.5 & 12.902 & 0.514 & $8.5 E-4$ \\
\hline 32 & 11 & 22 & 33 & 11 & 23 & 65194.420 & -0.068 & 0.10 & -0.5 & 18.099 & 0.622 & $1.1 \mathrm{E}-3$ \\
\hline 32 & 11 & 21 & 33 & 11 & 22 & 65194.420 & -0.068 & 0.10 & -0.5 & 18.099 & 0.622 & $1.1 \mathrm{E}-3$ \\
\hline 32 & 12 & 21 & 33 & 12 & 22 & 65199.900 & 0.021 & 0.10 & 0.2 & 23.792 & 0.741 & $1.1 E-3$ \\
\hline 32 & 12 & 20 & 33 & 12 & 21 & 65199.900 & 0.021 & 0.10 & 0.2 & 23.792 & 0.741 & 1. $1 \mathrm{E}-3$ \\
\hline 32 & 13 & 20 & 33 & 13 & 21 & 65205.590 & -0.243 & 0.10 & $-1 \cdot 8$ & 29.979 & 0.869 & 1. $0 E-3$ \\
\hline 32 & 13 & 19 & 33 & 13 & 20 & 65205.590 & -0.243 & 0.10 & -1.8 & 29.979 & 0.869 & 1. $0 E-3$ \\
\hline 32 & 14 & 19 & 33 & 14 & 20 & 65211.990 & -0.340 & 0.10 & -2.5 & 36.661 & 1.008 & $8 \cdot 3 E-4$ \\
\hline 32 & 14 & 18 & 33 & 14 & 19 & 65211.990 & -0.340 & 0.10 & -2.5 & 36.661 & 1.008 & $8 \cdot 3 E-4$ \\
\hline 32 & 15 & 18 & 33 & 15 & 19 & 65219.470 & 0.113 & 0.10 & 0.8 & 43.838 & 1.157 & $5.7 E-4$ \\
\hline 32 & 15 & 17 & 33 & 15 & 18 & 65219.470 & 0.113 & 0.10 & 0.8 & 43.838 & 1.157 & $5.7 E-4$ \\
\hline 33 & 7 & 27 & 34 & 7 & 28 & 67154.190 & 0.017 & 0.10 & 0.1 & -0.451 & 0.276 & $-2 \cdot 1 E-3$ \\
\hline 33 & 7 & 26 & 34 & 7 & 27 & 67154.190 & 0.017 & 0.10 & 0.1 & -0.451 & 0.276 & $-2 \cdot 1 E-3$ \\
\hline 33 & 8 & 26 & 34 & 8 & 27 & 67156.730 & -0.054 & 0.10 & -0.4 & 3.381 & 0.360 & $-6 \cdot 3 E-4$ \\
\hline 33 & 8 & 25 & 34 & 8 & 26 & 67156.730 & -0.054 & 0.10 & -0.4 & 3.381 & 0.360 & $-6 \cdot 3 E-4$ \\
\hline 33 & 9 & 25 & 34 & 9 & 26 & 67160.450 & 0.153 & 0.10 & 1.1 & 7.722 & 0.456 & 2. $9 \mathrm{E}-4$ \\
\hline 33 & 9 & 24 & 34 & 9 & 25 & 67160.450 & 0.153 & 0.10 & 1.1 & 7.722 & 0.456 & 2. $9 \mathrm{E}-4$ \\
\hline 33 & 10 & 24 & 34 & 10 & 25 & 67164.520 & -0.038 & 0.10 & -0.3 & 12.574 & 0.563 & 8. $3 E-4$ \\
\hline 33 & 10 & 23 & 34 & 10 & 24 & 67164.520 & -0.038 & 0.10 & -0.3 & 12.574 & 0.563 & 8. $3 E-4$ \\
\hline 33 & 11 & 23 & 34 & 11 & 24 & 67169.400 & -0.086 & 0.10 & -0.6 & 17.936 & 0.681 & 1. $1 E-3$ \\
\hline 33 & 11 & 22 & 34 & 11 & 23 & 67169.400 & -0.086 & 0.10 & -0.6 & 17.936 & 0.681 & $1.1 E-3$ \\
\hline 33 & 12 & 22 & 34 & 12 & 23 & 67175.120 & 0.091 & 0.10 & 0.7 & 23.809 & 0.810 & 1. $2 \mathrm{E}-3$ \\
\hline 33 & 12 & 21 & 34 & 12 & 22 & 67175.120 & 0.091 & 0.10 & 0.7 & 23.809 & 0.810 & 1. $2 E-3$ \\
\hline 33 & 13 & 21 & 34 & 13 & 22 & 67181.160 & 0.004 & 0.10 & 0.0 & 30.192 & 0.951 & 1. $2 E-3$ \\
\hline 33 & 13 & 20 & 34 & 13 & 21 & 67181.160 & 0.004 & 0.10 & 0.0 & 30.192 & 0.951 & 1. $2 E-3$ \\
\hline 33 & 14 & 20 & 34 & 14 & 21 & 67187.800 & -0.047 & 0.15 & -0.2 & 37.085 & 1.103 & 1. $0 E-3$ \\
\hline 33 & 14 & 19 & 34 & 14 & 20 & 67187.800 & -0.047 & 0.15 & -0.2 & 37.085 & 1.103 & 1. $0 E-3$ \\
\hline 33 & 15 & 19 & 34 & 15 & 20 & 67194.950 & -0.138 & 0.10 & -1.0 & 44.489 & 1.266 & 7. $6 \mathrm{E}-4$ \\
\hline 33 & 15 & 18 & 34 & 15 & 19 & 67194.950 & -0.138 & 0.10 & -1.0 & 44.489 & 1.266 & 7. $6 \mathrm{E}-4$ \\
\hline 33 & 16 & 18 & 34 & 16 & 19 & 67202.990 & 0.121 & 0.10 & 0.9 & 52.404 & 1.440 & 4. $4 \mathrm{E}-4$ \\
\hline 33 & 16 & 17 & 34 & 16 & 18 & 67202.990 & 0.121 & 0.10 & 0.9 & 52.404 & 1.440 & $4.4 E-4$ \\
\hline 33 & 17 & 17 & 34 & 17 & 18 & 67211.220 & 0.037 & 0.10 & 0.3 & 60.829 & 1.626 & $5.0 E-5$ \\
\hline 33 & 17 & 16 & 34 & 17 & 17 & 67211.220 & 0.037 & 0.10 & 0.3 & 60.829 & 1.626 & 5. $0 E-5$ \\
\hline 33 & 18 & 16 & 34 & 18 & 17 & 67220.350 & 0.323 & 0.10 & 2.5 & 69.765 & 1.823 & $-4.0 E-4$ \\
\hline 33 & 18 & 15 & 34 & 18 & 16 & 67220.350 & 0.323 & 0.10 & 2.5 & 69.765 & 1.823 & $-4.0 E-4$ \\
\hline 33 & 19 & 15 & 34 & 19 & 16 & 67229.800 & 0.405 & 0.10 & 3.1 & 79.211 & 2.031 & $-9.0 E-4$ \\
\hline 33 & 19 & 14 & 34 & 19 & 15 & 67229.800 & 0.405 & 0.10 & 3.1 & 79.211 & 2.031 & $-9.0 E-4$ \\
\hline
\end{tabular}









\begin{tabular}{|c|c|c|c|c|c|c|c|c|c|c|c|c|}
\hline 37 & 9 & 28 & 38 & 9 & 29 & 75058.870 & -0.011 & 0.10 & -0.1 & 5.148 & 0.636 & $-7.4 E-4$ \\
\hline 37 & 10 & 28 & 38 & 10 & 29 & 75063.710 & 0.200 & 0.10 & 1.5 & 10.601 & 0.785 & 4. $0 E-4$ \\
\hline 37 & 10 & 27 & 38 & 10 & 28 & 75063.710 & 0.200 & 0.10 & 1.5 & 10.601 & 0.785 & 4. $0 E-4$ \\
\hline 37 & 11 & 27 & 38 & 11 & 28 & 75068.980 & 0.050 & 0.10 & 0.4 & 16.627 & 0.950 & $1.1 E-3$ \\
\hline 37 & 11 & 26 & 38 & 11 & 27 & 75068.980 & 0.050 & 0.10 & 0.4 & 16.627 & 0.950 & 1. $1 E-3$ \\
\hline 37 & 12 & 26 & 38 & 12 & 27 & 75075.200 & 0.130 & 0.10 & 1.0 & 23.227 & 1.131 & 1. $5 E-3$ \\
\hline 37 & 12 & 25 & 38 & 12 & 26 & 75075.200 & 0.130 & 0.10 & 1.0 & 23.227 & 1.131 & 1. $5 E-3$ \\
\hline 37 & 13 & 25 & 38 & 13 & 26 & 75082.000 & 0.114 & 0.10 & 0.9 & 30.400 & 1.327 & 1. $7 E-3$ \\
\hline 37 & 13 & 24 & 38 & 13 & 25 & 75082.000 & 0.114 & 0.10 & 0.9 & 30.400 & 1.327 & $1.7 E-3$ \\
\hline 37 & 14 & 24 & 38 & 14 & 25 & 75089.230 & -0.120 & 0.10 & -0.9 & 38.147 & 1.539 & 1. $7 E-3$ \\
\hline 37 & 14 & 23 & 38 & 14 & 24 & 75089.230 & -0.120 & 0.10 & -0.9 & 38.147 & 1.539 & 1. $7 E-3$ \\
\hline 37 & 15 & 23 & 38 & 15 & 24 & 75097.480 & 0.037 & 0.10 & 0.3 & 46.468 & 1.767 & 1. $6 E-3$ \\
\hline 37 & 15 & 22 & 38 & 15 & 23 & 75097.480 & 0.037 & 0.10 & 0.3 & 46.468 & 1.767 & 1. $6 E-3$ \\
\hline 37 & 16 & 22 & 38 & 16 & 23 & 75105.970 & -0.181 & 0.10 & -1.4 & 55.363 & 2.011 & 1. $3 E-3$ \\
\hline 37 & 16 & 21 & 38 & 16 & 22 & 75105.970 & -0.181 & 0.10 & -1.4 & 55.363 & 2.011 & 1. $3 E-3$ \\
\hline 37 & 17 & 21 & 38 & 17 & 22 & 75115.310 & -0.155 & 0.10 & -1.2 & 64.831 & 2.270 & $9.9 \mathrm{E}-4$ \\
\hline 37 & 17 & 20 & 38 & 17 & 21 & 75115.310 & -0.155 & 0.10 & -1.2 & 64.831 & 2.270 & $9.9 E-4$ \\
\hline 38 & 8 & 31 & 39 & 8 & 32 & 77029.720 & 0.090 & 0.15 & 0.5 & -0.688 & 0.543 & $-3.2 E-3$ \\
\hline 38 & 8 & 30 & 39 & 8 & 31 & 77029.720 & 0.090 & 0.15 & 0.5 & -0.688 & 0.543 & $-3 \cdot 2 E-3$ \\
\hline 38 & 9 & 30 & 39 & 9 & 31 & 77033.670 & 0.276 & 0.10 & 2.1 & 4.328 & 0.688 & $-1 \cdot 2 E-3$ \\
\hline 38 & 9 & 29 & 39 & 9 & 30 & 77033.670 & 0.276 & 0.10 & 2.1 & 4.328 & 0.688 & $-1 \cdot 2 E-3$ \\
\hline 38 & 10 & 29 & 39 & 10 & 30 & 77038.000 & -0.108 & 0.10 & -0.8 & 9.932 & 0.849 & 1. $7 E-4$ \\
\hline 38 & 10 & 28 & 39 & 10 & 29 & 77038.000 & -0.108 & 0.10 & -0.8 & 9.932 & 0.849 & 1. $7 E-4$ \\
\hline 38 & 11 & 28 & 39 & 11 & 29 & 77043.840 & 0.194 & 0.10 & 1.5 & 16.126 & 1.027 & 1. $0 E-3$ \\
\hline 38 & 11 & 27 & 39 & 11 & 28 & 77043.840 & 0.194 & 0.10 & 1.5 & 16.126 & 1.027 & 1. $0 \mathrm{E}-3$ \\
\hline 38 & 12 & 27 & 39 & 12 & 28 & 77049.870 & -0.062 & 0.10 & -0.5 & 22.910 & 1.223 & 1. $5 E-3$ \\
\hline 38 & 12 & 26 & 39 & 12 & 27 & 77049.870 & -0.062 & 0.10 & -0.5 & 22.910 & 1.223 & 1. $5 E-3$ \\
\hline 38 & 13 & 26 & 39 & 13 & 27 & 77056.700 & -0.219 & 0.10 & $-1 \cdot 6$ & 30.283 & 1.435 & 1. $8 E-3$ \\
\hline 38 & 13 & 25 & 39 & 13 & 26 & 77056.700 & -0.219 & 0.10 & -1.6 & 30.283 & 1.435 & 1. $8 \mathrm{E}-3$ \\
\hline 38 & 14 & 25 & 39 & 14 & 26 & 77064.520 & -0.056 & 0.10 & -0.4 & 38.246 & 1.664 & $1.8 E-3$ \\
\hline 38 & 14 & 24 & 39 & 14 & 25 & 77064.520 & -0.056 & 0.10 & -0.4 & 38.246 & 1.664 & 1. $8 E-3$ \\
\hline 38 & 15 & 24 & 39 & 15 & 25 & 77072.840 & -0.042 & 0.10 & -0.3 & 46.798 & 1.910 & 1. $8 E-3$ \\
\hline 38 & 15 & 23 & 39 & 15 & 24 & 77072.840 & -0.042 & 0.10 & -0.3 & 46.798 & 1.910 & 1. $8 E-3$ \\
\hline 39 & 8 & 32 & 40 & 8 & 33 & 79003.940 & -0.108 & 0.10 & -0.8 & -1.720 & 0.586 & $-4.0 E-3$ \\
\hline 39 & 8 & 31 & 40 & 8 & 32 & 79003.940 & -0.108 & 0.10 & -0.8 & -1.720 & 0.586 & $-4.0 E-3$ \\
\hline 39 & 9 & 31 & 40 & 9 & 32 & 79008.020 & 0.170 & 0.10 & 1.3 & 3.433 & 0.742 & $-1 \cdot 6 E-3$ \\
\hline 39 & 9 & 30 & 40 & 9 & 31 & 79008.020 & 0.170 & 0.10 & 1.3 & 3.433 & 0.742 & $-1 \cdot 6 E-3$ \\
\hline 39 & 10 & 30 & 40 & 10 & 31 & 79012.730 & 0.083 & 0.10 & 0.6 & 9.190 & 0.916 & $-1 \cdot 0 E-4$ \\
\hline 39 & 10 & 29 & 40 & 10 & 30 & 79012.730 & 0.083 & 0.10 & 0.6 & 9.190 & 0.916 & $-1.0 E-4$ \\
\hline 39 & 11 & 29 & 40 & 11 & 30 & 79018.450 & 0.148 & 0.10 & 1.1 & 15.553 & 1.108 & 9. $0 \mathrm{E}-4$ \\
\hline 39 & 11 & 28 & 40 & 11 & 29 & 79018.450 & 0.148 & 0.10 & 1.1 & 15.553 & 1.108 & 9. $0 E-4$ \\
\hline 39 & 12 & 28 & 40 & 12 & 29 & 79024.780 & 0.047 & 0.10 & 0.4 & 22.521 & 1.319 & 1. $5 \mathrm{E}-3$ \\
\hline 39 & 12 & 27 & 40 & 12 & 28 & 79024.780 & 0.047 & 0.10 & 0.4 & 22.521 & 1.319 & $1.5 E-3$ \\
\hline 39 & 13 & 27 & 40 & 13 & 28 & 79031.910 & 0.020 & 0.10 & 0.2 & 30.094 & 1.548 & 1. $9 E-3$ \\
\hline 39 & 13 & 26 & 40 & 13 & 27 & 79031.910 & 0.020 & 0.10 & 0.2 & 30.094 & 1.548 & 1. $9 E-3$ \\
\hline 39 & 15 & 25 & 40 & 15 & 26 & 79048.170 & -0.088 & 0.10 & -0.7 & 47.058 & 2.061 & 2. $0 E-3$ \\
\hline 39 & 15 & 24 & 40 & 15 & 25 & 79048.170 & -0.088 & 0.10 & -0.7 & 47.058 & 2.061 & 2. $0 E-3$ \\
\hline 39 & 17 & 23 & 40 & 17 & 24 & 79067.170 & -0.077 & 0.10 & -0.6 & 66.444 & 2.647 & 1. $5 \mathrm{E}-3$ \\
\hline 39 & 17 & 22 & 40 & 17 & 23 & 79067.170 & -0.077 & 0.10 & -0.6 & 66.444 & 2.647 & 1. $5 E-3$ \\
\hline 39 & 18 & 22 & 40 & 18 & 23 & 79077.800 & 0.102 & 0.10 & 0.9 & 77.046 & 2.968 & 1.1E-3 \\
\hline 39 & 18 & 21 & 40 & 18 & 22 & 79077.800 & 0.102 & 0.10 & 0.9 & 77.046 & 2.968 & 1. $1 E-3$ \\
\hline
\end{tabular}

rms deviation: 1.3476

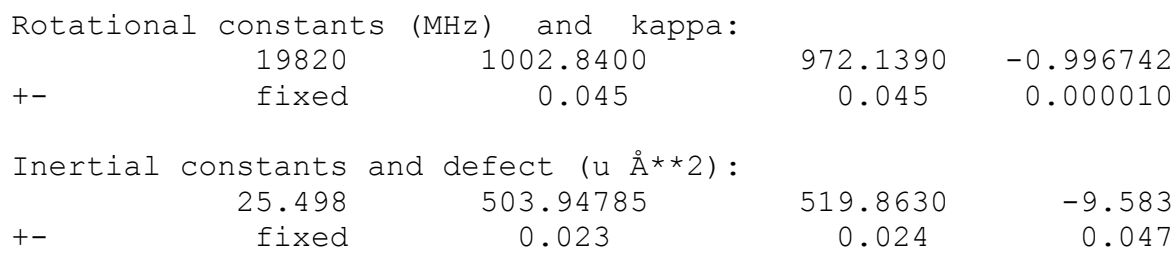




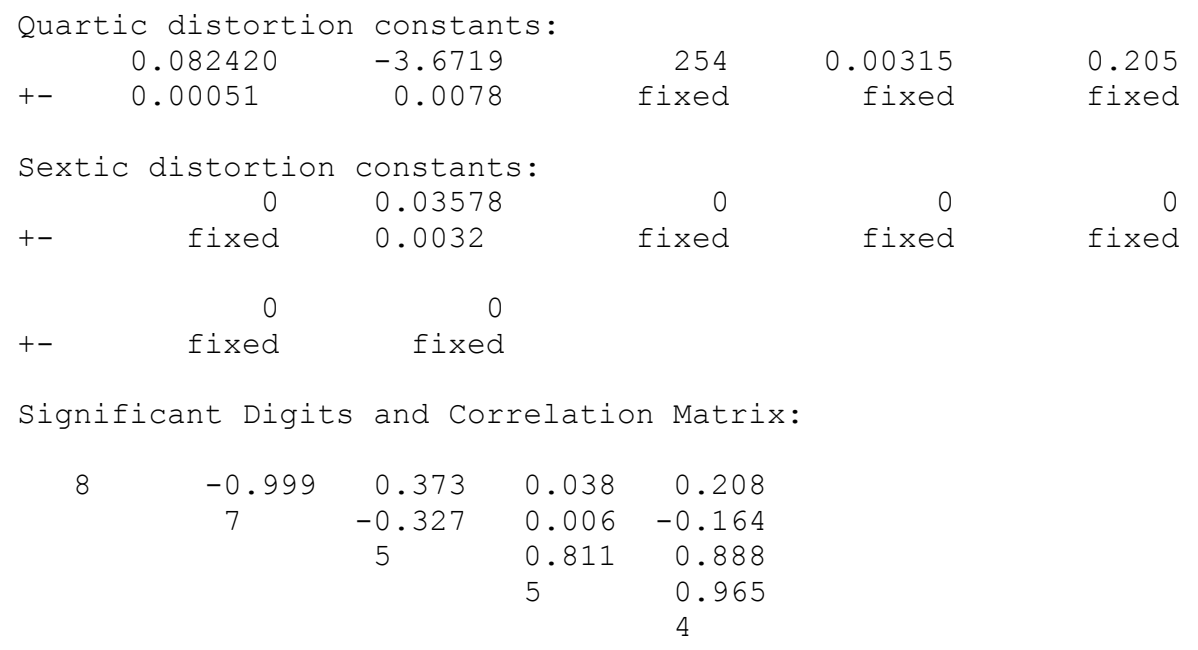


TABLE 2S: Microwave Spectrum of the Ground Vibrational state of Conformer II of $\mathrm{H}^{78} \mathrm{SeCH}_{2} \mathrm{CH}_{2} \mathrm{C} \equiv \mathrm{CH}$

Total number of transitions used in the least-squares fit: 118

\begin{tabular}{|c|c|c|c|c|c|c|c|c|c|c|c|}
\hline & \multicolumn{4}{|c|}{ Transition } & \multicolumn{2}{|c|}{ obs. frequency } & $\begin{array}{l}\text { obs.- } \\
\text { calc. }\end{array}$ & weight & t & \multicolumn{2}{|c|}{$\begin{array}{c}\text { distortion corre } \\
\text { total fixed }\end{array}$} \\
\hline 20 & 9 & 12 & 21 & 9 & 13 & 41810.920 & -0.032 & 0.10 & -0.3 & 9.903 & 2. $0 E-4$ \\
\hline 20 & 9 & 11 & 21 & 9 & 12 & 41810.920 & -0.032 & 0.10 & -0.3 & 9.903 & $2.0 E-4$ \\
\hline 20 & 10 & 11 & 21 & 10 & 12 & 41813.880 & 0.062 & 0.10 & 0.6 & 12.901 & $8.4 E-5$ \\
\hline 20 & 10 & 10 & 21 & 10 & 11 & 41813.880 & 0.062 & 0.10 & 0.6 & 12.901 & $8.4 E-5$ \\
\hline 20 & 11 & 10 & 21 & 11 & 11 & 41817.200 & 0.166 & 0.10 & 1.6 & 16.215 & $-7.5 E-5$ \\
\hline 20 & 11 & 9 & 21 & 11 & 10 & 41817.200 & 0.166 & 0.10 & 1.6 & 16.215 & $-7.5 E-5$ \\
\hline 21 & 8 & 14 & 22 & 8 & 15 & 43799.230 & 0.100 & 0.10 & 1.0 & 7.271 & $3.2 E-4$ \\
\hline 21 & 8 & 13 & 22 & 8 & 14 & 43799.230 & 0.100 & 0.10 & 1.0 & 7.271 & 3. $2 E-4$ \\
\hline 22 & 10 & 13 & 23 & 10 & 14 & 45795.610 & 0.031 & 0.20 & 0.1 & 13.501 & $2.6 E-4$ \\
\hline 22 & 10 & 12 & 23 & 10 & 13 & 45795.610 & 0.031 & 0.20 & 0.1 & 13.501 & 2. $6 E-4$ \\
\hline 22 & 13 & 10 & 23 & 13 & 11 & 45806.890 & -0.312 & 0.20 & -1.5 & 25.425 & $-3.1 E-4$ \\
\hline 22 & 13 & 9 & 23 & 13 & 10 & 45806.890 & -0.312 & 0.20 & -1.5 & 25.425 & $-3.1 E-4$ \\
\hline 23 & 8 & 16 & 24 & 8 & 17 & 47780.450 & 0.058 & 0.12 & 0.5 & 7.246 & $4.1 E-4$ \\
\hline 23 & 8 & 15 & 24 & 8 & 16 & 47780.450 & 0.058 & 0.12 & 0.5 & 7.246 & $4.1 E-4$ \\
\hline 23 & 9 & 15 & 24 & 9 & 16 & 47783.310 & 0.130 & 0.15 & 0.8 & 10.312 & 4. $2 E-4$ \\
\hline 23 & 9 & 14 & 24 & 9 & 15 & 47783.310 & 0.130 & 0.15 & 0.8 & 10.312 & $4.2 \mathrm{E}-4$ \\
\hline 24 & 8 & 17 & 25 & 8 & 18 & 49771.020 & 0.043 & 0.20 & 0.2 & 7.167 & $4.4 E-4$ \\
\hline 24 & 8 & 16 & 25 & 8 & 17 & 49771.020 & 0.043 & 0.20 & 0.2 & 7.167 & 4. $4 E-4$ \\
\hline 25 & 7 & 19 & 26 & 7 & 20 & 51759.140 & 0.022 & 0.20 & 0.1 & 4.111 & $2.0 E-4$ \\
\hline 25 & 7 & 18 & 26 & 7 & 19 & 51759.140 & 0.022 & 0.20 & 0.1 & 4.111 & 2. $0 E-4$ \\
\hline 25 & 8 & 18 & 26 & 8 & 19 & 51761.640 & 0.110 & 0.15 & 0.7 & 7.042 & 4. $6 E-4$ \\
\hline 25 & 8 & 17 & 26 & 8 & 18 & 51761.640 & 0.110 & 0.15 & 0.7 & 7.042 & 4. $6 E-4$ \\
\hline 26 & 7 & 20 & 27 & 7 & 21 & 53749.730 & 0.143 & 0.15 & 0.9 & 3.824 & 1. $1 E-4$ \\
\hline 26 & 7 & 19 & 27 & 7 & 20 & 53749.730 & 0.143 & 0.15 & 0.9 & 3.824 & $1.1 E-4$ \\
\hline 26 & 8 & 19 & 27 & 8 & 20 & 53752.090 & 0.042 & 0.15 & 0.3 & 6.868 & 4. $6 E-4$ \\
\hline 26 & 8 & 18 & 27 & 8 & 19 & 53752.090 & 0.042 & 0.15 & 0.3 & 6.868 & 4. $6 E-4$ \\
\hline 26 & 9 & 18 & 27 & 9 & 19 & 53755.270 & 0.168 & 0.15 & 1.1 & 10.317 & $6.1 E-4$ \\
\hline 26 & 9 & 17 & 27 & 9 & 18 & 53755.270 & 0.168 & 0.15 & 1.1 & 10.317 & $6.1 \mathrm{E}-4$ \\
\hline 26 & 11 & 16 & 27 & 11 & 17 & 53762.570 & -0.158 & 0.12 & $-1 \cdot 3$ & 18.432 & $5.4 E-4$ \\
\hline 26 & 11 & 15 & 27 & 11 & 16 & 53762.570 & -0.158 & 0.12 & $-1 \cdot 3$ & 18.432 & $5.4 E-4$ \\
\hline 28 & 8 & 21 & 29 & 8 & 22 & 57732.800 & -0.179 & 0.10 & -1.7 & 6.367 & 3. $9 E-4$ \\
\hline 28 & 8 & 20 & 29 & 8 & 21 & 57732.800 & -0.179 & 0.10 & -1.7 & 6.367 & 3. $9 E-4$ \\
\hline 28 & 10 & 19 & 29 & 10 & 20 & 57739.820 & -0.165 & 0.10 & -1.6 & 14.212 & $7.8 E-4$ \\
\hline 28 & 10 & 18 & 29 & 10 & 19 & 57739.820 & -0.165 & 0.10 & -1.6 & 14.212 & 7. $8 E-4$ \\
\hline 30 & 7 & 24 & 31 & 7 & 25 & 61711.230 & 0.085 & 0.10 & 0.8 & 2.155 & $-6.6 E-4$ \\
\hline 30 & 7 & 24 & 31 & 7 & 25 & 61711.230 & 0.085 & 0.10 & 0.8 & 2.155 & $-6.6 E-4$ \\
\hline 30 & 8 & 23 & 31 & 8 & 24 & 61713.620 & -0.139 & 0.10 & $-1 \cdot 3$ & 5.650 & 1. $9 E-4$ \\
\hline 30 & 8 & 22 & 31 & 8 & 23 & 61713.620 & -0.139 & 0.10 & $-1 \cdot 3$ & 5.650 & 1. $9 E-4$ \\
\hline 30 & 9 & 22 & 31 & 9 & 23 & 61717.220 & 0.099 & 0.10 & 1.0 & 9.610 & $6.7 E-4$ \\
\hline 30 & 9 & 21 & 31 & 9 & 22 & 61717.220 & 0.099 & 0.10 & 1.0 & 9.610 & $6.7 E-4$ \\
\hline 30 & 10 & 21 & 31 & 10 & 22 & 61721.070 & -0.051 & 0.10 & -0.5 & 14.036 & $9.0 \mathrm{E}-4$ \\
\hline 30 & 10 & 20 & 31 & 10 & 21 & 61721.070 & -0.051 & 0.10 & -0.5 & 14.036 & $9.0 E-4$ \\
\hline 30 & 11 & 20 & 31 & 11 & 21 & 61725.670 & -0.029 & 0.10 & -0.3 & 18.928 & $9.7 E-4$ \\
\hline 30 & 11 & 19 & 31 & 11 & 20 & 61725.670 & -0.029 & 0.10 & -0.3 & 18.928 & $9.7 E-4$ \\
\hline 30 & 12 & 19 & 31 & 12 & 20 & 61730.670 & -0.148 & 0.10 & $-1 \cdot 4$ & 24.285 & $9.1 E-4$ \\
\hline 30 & 12 & 18 & 31 & 12 & 19 & 61730.670 & -0.148 & 0.10 & -1.4 & 24.285 & $9.1 \mathrm{E}-4$ \\
\hline 30 & 13 & 18 & 31 & 13 & 19 & 61736.390 & -0.067 & 0.10 & -0.7 & 30.108 & 7. $6 E-4$ \\
\hline 30 & 13 & 17 & 31 & 13 & 18 & 61736.390 & -0.067 & 0.10 & -0.7 & 30.108 & 7. $6 \mathrm{E}-4$ \\
\hline 30 & 15 & 16 & 31 & 15 & 17 & 61749.220 & -0.016 & 0.10 & -0.2 & 43.152 & $2.4 E-4$ \\
\hline 30 & 15 & 15 & 31 & 15 & 16 & 61749.220 & -0.016 & 0.10 & -0.2 & 43.152 & $2.4 E-4$ \\
\hline
\end{tabular}




\begin{tabular}{|c|c|c|c|c|c|c|c|c|c|c|}
\hline 32 & 26 & 33 & 7 & 27 & 65691.670 & -0.050 & 0.10 & -0.5 & 0.980 & $-1.4 E-3$ \\
\hline 32 & 25 & 33 & 7 & 26 & 65691.670 & -0.050 & 0.10 & -0.5 & 0.980 & $-1 \cdot 4 E-3$ \\
\hline 32 & 24 & 33 & 9 & 25 & 65698.030 & 0.157 & 0.10 & 1.5 & 8.917 & $5.5 E-4$ \\
\hline 32 & 23 & 33 & 9 & 24 & 65698.030 & 0.157 & 0.10 & 1.5 & 8.917 & $5.5 E-4$ \\
\hline 33 & 25 & 34 & 9 & 26 & 67688.280 & 0.101 & 0.10 & 1.0 & 8.479 & $4.5 E-4$ \\
\hline 33 & 24 & 34 & 9 & 25 & 67688.280 & 0.101 & 0.10 & 1.0 & 8.479 & $4.5 E-4$ \\
\hline $33 \quad 10$ & 24 & 34 & 10 & 25 & 67692.450 & -0.021 & 0.10 & -0.2 & 13.334 & $9.6 E-4$ \\
\hline 3310 & 23 & 34 & 10 & 24 & 67692.450 & -0.021 & 0.10 & -0.2 & 13.334 & $9.6 E-4$ \\
\hline 34 & 29 & 35 & 6 & 30 & 69670.690 & -0.012 & 0.10 & -0.1 & -3.864 & $-5 \cdot 3 E-3$ \\
\hline 34 & 28 & 35 & 6 & 29 & 69670.690 & -0.012 & 0.10 & -0.1 & -3.864 & $-5 \cdot 3 E-3$ \\
\hline 7 & 28 & 35 & 7 & 29 & 69672.060 & -0.087 & 0.20 & -0.4 & -0.441 & $-2 \cdot 5 E-3$ \\
\hline 34 & 27 & 35 & 7 & 28 & 69672.060 & -0.087 & 0.20 & -0.4 & -0.441 & $-2.5 E-3$ \\
\hline 34 & 26 & 35 & 9 & 27 & 69678.450 & 0.013 & 0.10 & 0.1 & 7.978 & 2. $9 E-4$ \\
\hline 34 & 25 & 35 & 9 & 26 & 69678.450 & 0.013 & 0.10 & 0.1 & 7.978 & 2. $9 E-4$ \\
\hline $34 \quad 10$ & 25 & 35 & 10 & 26 & 69682.750 & -0.071 & 0.10 & -0.7 & 12.975 & $9.2 \mathrm{E}-4$ \\
\hline 10 & 24 & 35 & 10 & 25 & 69682.750 & -0.071 & 0.10 & -0.7 & 12.975 & $9.2 \mathrm{E}-4$ \\
\hline 11 & 24 & 35 & 11 & 25 & 69687.620 & -0.273 & 0.10 & -2.6 & 18.499 & 1. $3 E-3$ \\
\hline 11 & 23 & 35 & 11 & 24 & 69687.620 & -0.273 & 0.10 & -2.6 & 18.499 & 1. $3 E-3$ \\
\hline 12 & 23 & 35 & 12 & 24 & 69693.550 & -0.049 & 0.10 & -0.5 & 24.548 & 1. $4 E-3$ \\
\hline 12 & 22 & 35 & 12 & 23 & 69693.550 & -0.049 & 0.10 & -0.5 & 24.548 & $1.4 \mathrm{E}-3$ \\
\hline 13 & 22 & 35 & 13 & 23 & 69699.990 & 0.082 & 0.10 & 0.8 & 31.122 & 1. $4 \mathrm{E}-3$ \\
\hline 13 & 21 & 35 & 13 & 22 & 69699.990 & 0.082 & 0.10 & 0.8 & 31.122 & $1.4 E-3$ \\
\hline 14 & 21 & 35 & 14 & 22 & 69706.740 & -0.058 & 0.10 & -0.6 & 38.223 & 1. $3 E-3$ \\
\hline 14 & 20 & 35 & 14 & 21 & 69706.740 & -0.058 & 0.10 & -0.6 & 38.223 & 1. $3 E-3$ \\
\hline 15 & 20 & 35 & 15 & 21 & 69714.520 & 0.265 & 0.10 & 2.7 & 45.849 & 1. $0 \mathrm{E}-3$ \\
\hline 15 & 19 & 35 & 15 & 20 & 69714.520 & 0.265 & 0.10 & 2.7 & 45.849 & 1. $0 E-3$ \\
\hline 6 & 30 & 36 & 6 & 31 & 71660.970 & 0.036 & 0.10 & 0.4 & -4.770 & $-6.5 E-3$ \\
\hline 6 & 29 & 36 & 6 & 30 & 71660.970 & 0.036 & 0.10 & 0.4 & -4.770 & $-6.5 E-3$ \\
\hline 8 & 28 & 36 & 8 & 29 & 71664.930 & -0.053 & 0.10 & -0.5 & 2.811 & $-1 \cdot 1 E-3$ \\
\hline 8 & 27 & 36 & 8 & 28 & 71664.930 & -0.053 & 0.10 & -0.5 & 2.811 & $-1.1 E-3$ \\
\hline 9 & 27 & 36 & 9 & 28 & 71668.820 & 0.175 & 0.10 & 1.7 & 7.411 & $9.6 E-5$ \\
\hline 35 & 26 & 36 & 9 & 27 & 71668.820 & 0.175 & 0.10 & 1.7 & 7.411 & $9.6 E-5$ \\
\hline 510 & 26 & 36 & 10 & 27 & 71673.070 & -0.048 & 0.10 & -0.5 & 12.552 & $8.6 E-4$ \\
\hline 510 & 25 & 36 & 10 & 26 & 71673.070 & -0.048 & 0.10 & -0.5 & 12.552 & 8. $6 E-4$ \\
\hline 68 & 29 & 37 & 8 & 30 & 73655.100 & 0.008 & 0.10 & 0.1 & 2.049 & $-1 \cdot 6 E-3$ \\
\hline 8 & 28 & 37 & 8 & 29 & 73655.100 & 0.008 & 0.10 & 0.1 & 2.049 & $-1 \cdot 6 E-3$ \\
\hline 10 & 27 & 37 & 10 & 28 & 73663.450 & 0.089 & 0.10 & 0.9 & 12.061 & 7. $6 E-4$ \\
\hline 10 & 26 & 37 & 10 & 27 & 73663.450 & 0.089 & 0.10 & 0.9 & 12.061 & 7. $6 \mathrm{E}-4$ \\
\hline 12 & 25 & 37 & 12 & 26 & 73674.590 & -0.067 & 0.10 & -0.6 & 24.295 & 1. $6 \mathrm{E}-3$ \\
\hline 612 & 24 & 37 & 12 & 25 & 73674.590 & -0.067 & 0.10 & -0.6 & 24.295 & 1. $6 E-3$ \\
\hline 78 & 30 & 38 & 8 & 31 & 75645.120 & -0.034 & 0.10 & -0.3 & 1.218 & $-2 \cdot 2 E-3$ \\
\hline 8 & 29 & 38 & 8 & 30 & 75645.120 & -0.034 & 0.10 & -0.3 & 1.218 & $-2 \cdot 2 E-3$ \\
\hline 37 & 29 & 38 & 9 & 30 & 75648.880 & -0.027 & 0.10 & -0.3 & 6.074 & $-4.7 E-4$ \\
\hline 37 & 28 & 38 & 9 & 29 & 75648.880 & -0.027 & 0.10 & -0.3 & 6.074 & $-4.7 E-4$ \\
\hline $37 \quad 10$ & 28 & 38 & 10 & 29 & 75653.450 & -0.099 & 0.10 & -1.0 & 11.501 & $6.2 E-4$ \\
\hline 3710 & 27 & 38 & 10 & 28 & 75653.450 & -0.099 & 0.10 & -1.0 & 11.501 & $6.2 E-4$ \\
\hline $37 \quad 11$ & 27 & 38 & 11 & 28 & 75658.970 & 0.002 & 0.10 & 0.0 & 17.498 & 1. $3 E-3$ \\
\hline $37 \quad 11$ & 26 & 38 & 11 & 27 & 75658.970 & 0.002 & 0.10 & 0.0 & 17.498 & 1. $3 E-3$ \\
\hline $37 \quad 12$ & 26 & 38 & 12 & 27 & 75665.090 & -0.007 & 0.10 & -0.1 & 24.066 & 1. $7 E-3$ \\
\hline $37 \quad 12$ & 25 & 38 & 12 & 26 & 75665.090 & -0.007 & 0.10 & -0.1 & 24.066 & $1.7 E-3$ \\
\hline $37 \quad 13$ & 25 & 38 & 13 & 26 & 75671.940 & 0.045 & 0.10 & 0.4 & 31.204 & 1. $8 E-3$ \\
\hline $37 \quad 13$ & 24 & 38 & 13 & 25 & 75671.940 & 0.045 & 0.10 & 0.4 & 31.204 & $1.8 E-3$ \\
\hline $37 \quad 14$ & 24 & 38 & 14 & 25 & 75679.240 & -0.095 & 0.10 & -0.9 & 38.913 & $1.8 E-3$ \\
\hline $37 \quad 14$ & 23 & 38 & 14 & 24 & 75679.240 & -0.095 & 0.10 & -0.9 & 38.913 & $1.8 E-3$ \\
\hline 38 & 31 & 39 & 8 & 32 & 77635.050 & -0.116 & 0.10 & -1.1 & 0.316 & $-2.8 E-3$ \\
\hline 38 & 30 & 39 & 8 & 31 & 77635.050 & -0.116 & 0.10 & -1.1 & 0.316 & $-2.8 E-3$ \\
\hline 38 & 30 & 39 & 9 & 31 & 77639.030 & 0.072 & 0.10 & 0.7 & 5.301 & $-8.5 E-4$ \\
\hline 38 & 29 & 39 & 9 & 30 & 77639.030 & 0.072 & 0.10 & 0.7 & 5.301 & $-8.5 E-4$ \\
\hline $38 \quad 10$ & 29 & 39 & 10 & 30 & 77643.770 & 0.091 & 0.10 & 0.9 & 10.870 & 4. $2 E-4$ \\
\hline $38 \quad 10$ & 28 & 39 & 10 & 29 & 77643.770 & 0.091 & 0.10 & 0.9 & 10.870 & $4.2 E-4$ \\
\hline $\begin{array}{ll}38 & 11\end{array}$ & 28 & 39 & 11 & 29 & 77649.430 & 0.221 & 0.10 & 2.2 & 17.025 & 1. $2 \mathrm{E}-3$ \\
\hline $38 \quad 11$ & 27 & 39 & 11 & 28 & 77649.430 & 0.221 & 0.10 & 2.2 & 17.025 & 1. $2 E-3$ \\
\hline
\end{tabular}




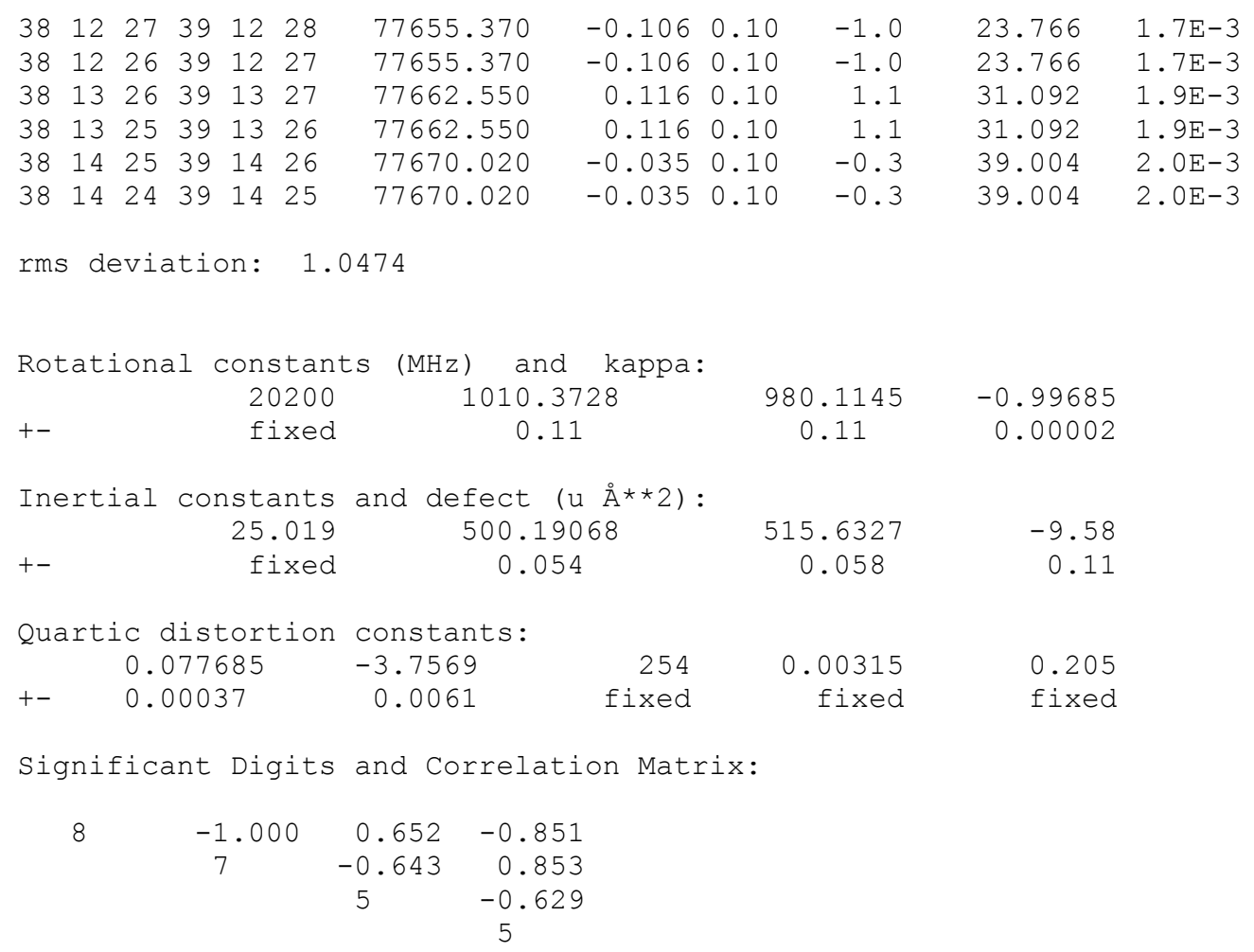


TABLE 3S: Microwave Spectrum of the Ground Vibrational State of Conformer IV of $\mathrm{H}^{80} \mathrm{SeCH}_{2} \mathrm{CH}_{2} \mathrm{C} \equiv \mathrm{CH}$

Total number of transitions used in the least-squares fit: 468

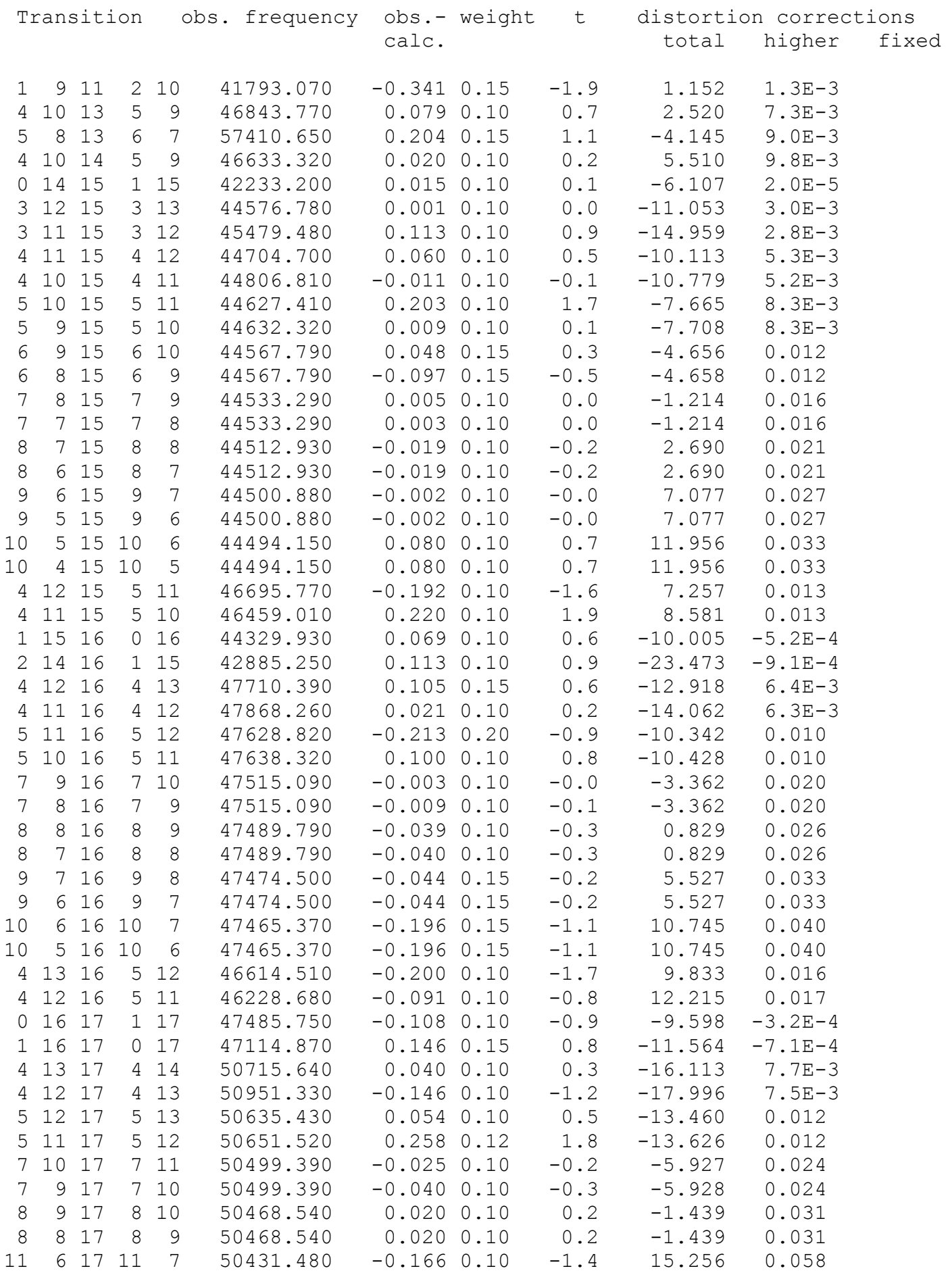




\begin{tabular}{|c|c|c|c|c|c|c|c|c|c|c|c|}
\hline 16 & 11 & 5 & 17 & 11 & 6 & 50431.480 & -0.166 & 0.10 & $-1 \cdot 4$ & 15.256 & 0.058 \\
\hline 17 & 4 & 13 & 17 & 5 & 12 & 45928.620 & 0.063 & 0.10 & 0.5 & 16.585 & 0.021 \\
\hline 17 & 0 & 17 & 18 & 1 & 18 & 50144.820 & 0.236 & 0.10 & 2.0 & -11.650 & $-5.9 E-4$ \\
\hline 17 & 1 & 17 & 18 & 0 & 18 & 49879.620 & -0.245 & 0.12 & -1.7 & $-13 \cdot 332$ & $-9.6 E-4$ \\
\hline 17 & 2 & 16 & 18 & 1 & 17 & 49805.790 & 0.133 & 0.10 & 1.1 & -27.639 & $-5 \cdot 2 E-4$ \\
\hline 17 & 3 & 15 & 18 & 2 & 16 & 40564.210 & 0.093 & 0.10 & 0.8 & -55.099 & $-6.5 E-3$ \\
\hline 17 & 4 & 13 & 18 & 4 & 14 & 54060.040 & 0.196 & 0.10 & 1.6 & -22.675 & $8.9 E-3$ \\
\hline 17 & 5 & 12 & 18 & 5 & 13 & 53672.730 & 0.120 & 0.10 & 1.0 & -17.357 & 0.014 \\
\hline 17 & 7 & 11 & 18 & 7 & 12 & 53486.690 & 0.273 & 0.15 & 1.5 & -8.941 & 0.028 \\
\hline 17 & 7 & 10 & 18 & 7 & 11 & 53486.690 & 0.241 & 0.15 & 1.3 & -8.941 & 0.028 \\
\hline 17 & 8 & 10 & 18 & 8 & 11 & 53449.050 & -0.085 & 0.10 & -0.7 & -4.145 & 0.037 \\
\hline 17 & 8 & 9 & 18 & 8 & 10 & 53449.050 & -0.086 & 0.10 & -0.7 & -4.145 & 0.037 \\
\hline 17 & 9 & 9 & 18 & 9 & 10 & 53425.950 & -0.017 & 0.10 & -0.1 & 1.195 & 0.046 \\
\hline 17 & 9 & 8 & 18 & 9 & 9 & 53425.950 & -0.017 & 0.10 & -0.1 & 1.195 & 0.046 \\
\hline 17 & 10 & 8 & 18 & 10 & 9 & 53411.470 & -0.171 & 0.10 & $-1 \cdot 4$ & 7.105 & 0.057 \\
\hline 17 & 10 & 7 & 18 & 10 & 8 & 53411.470 & -0.171 & 0.10 & $-1 \cdot 4$ & 7.105 & 0.057 \\
\hline 17 & 11 & 7 & 18 & 11 & 8 & 53403.070 & -0.172 & 0.10 & $-1 \cdot 4$ & 13.601 & 0.069 \\
\hline 17 & 11 & 6 & 18 & 11 & 7 & 53403.070 & -0.172 & 0.10 & $-1 \cdot 4$ & 13.601 & 0.069 \\
\hline 18 & 4 & 15 & 18 & 5 & 14 & 46461.540 & -0.244 & 0.10 & -2.1 & 15.139 & 0.026 \\
\hline 18 & 4 & 14 & 18 & 5 & 13 & 45541.460 & 0.138 & 0.10 & 1.2 & 21.904 & 0.026 \\
\hline 18 & 5 & 14 & 18 & 6 & 13 & 57050.320 & -0.056 & 0.10 & -0.5 & 12.226 & 0.032 \\
\hline 18 & 5 & 13 & 18 & 6 & 12 & 56990.790 & -0.051 & 0.12 & -0.4 & 12.832 & 0.032 \\
\hline 18 & 0 & 18 & 19 & 1 & 19 & 52817.940 & -0.021 & 0.10 & -0.2 & $-13 \cdot 912$ & $-9.6 E-4$ \\
\hline 18 & 1 & 18 & 19 & 0 & 19 & 52630.200 & -0.044 & 0.10 & -0.4 & $-15 \cdot 327$ & $-1 \cdot 3 E-3$ \\
\hline 18 & 2 & 17 & 19 & 1 & 18 & 53084.890 & 0.132 & 0.10 & 1.1 & -29.477 & $-1 \cdot 3 E-4$ \\
\hline 18 & 4 & 14 & 19 & 4 & 15 & 57196.150 & 0.121 & 0.15 & 0.7 & -28.186 & 0.010 \\
\hline 18 & 5 & 14 & 19 & 5 & 15 & 56660.980 & 0.040 & 0.10 & 0.3 & -21.143 & 0.017 \\
\hline 18 & 5 & 13 & 19 & 5 & 14 & 56703.670 & -0.065 & 0.10 & -0.5 & -21.684 & 0.017 \\
\hline 18 & 6 & 13 & 19 & 6 & 14 & 56549.040 & 0.127 & 0.10 & 1.1 & -17.055 & 0.024 \\
\hline 18 & 6 & 12 & 19 & 6 & 13 & 56550.950 & -0.065 & 0.10 & -0.5 & -17.088 & 0.024 \\
\hline 18 & 7 & 12 & 19 & 7 & 13 & 56476.400 & 0.134 & 0.10 & 1.1 & -12.435 & 0.033 \\
\hline 18 & 7 & 11 & 19 & 7 & 12 & 56476.400 & 0.068 & 0.10 & 0.6 & -12.436 & 0.033 \\
\hline 18 & 8 & 11 & 19 & 8 & 12 & 56431.800 & 0.006 & 0.10 & 0.1 & -7.317 & 0.043 \\
\hline 18 & 8 & 10 & 19 & 8 & 11 & 56431.800 & 0.005 & 0.10 & 0.0 & -7.317 & 0.043 \\
\hline 18 & 9 & 10 & 19 & 9 & 11 & 56403.940 & 0.045 & 0.10 & 0.4 & -1.643 & 0.055 \\
\hline 18 & 9 & 9 & 19 & 9 & 10 & 56403.940 & 0.045 & 0.10 & 0.4 & -1.643 & 0.055 \\
\hline 18 & 10 & 9 & 19 & 10 & 10 & 56386.210 & -0.132 & 0.10 & -1.1 & 4.622 & 0.067 \\
\hline 18 & 10 & 8 & 19 & 10 & 9 & 56386.210 & -0.132 & 0.10 & $-1 \cdot 1$ & 4.622 & 0.067 \\
\hline 18 & 11 & 8 & 19 & 11 & 9 & 56375.480 & -0.213 & 0.15 & -1.2 & 11.498 & 0.082 \\
\hline 18 & 11 & 7 & 19 & 11 & 8 & 56375.480 & -0.213 & 0.15 & $-1 \cdot 2$ & 11.498 & 0.082 \\
\hline 18 & 12 & 7 & 19 & 12 & 8 & 56370.120 & 0.207 & 0.20 & 0.9 & 18.997 & 0.097 \\
\hline 18 & 12 & 6 & 19 & 12 & 7 & 56370.120 & 0.207 & 0.20 & 0.9 & 18.997 & 0.097 \\
\hline 19 & 4 & 16 & 19 & 5 & 15 & 46404.660 & -0.104 & 0.10 & -0.9 & 17.684 & 0.032 \\
\hline 19 & 5 & 15 & 19 & 6 & 14 & 56938.190 & -0.159 & 0.10 & $-1 \cdot 3$ & 16.313 & 0.039 \\
\hline 19 & 5 & 14 & 19 & 6 & 13 & 56838.150 & 0.029 & 0.10 & 0.2 & 17.427 & 0.039 \\
\hline 19 & 1 & 19 & 20 & 0 & 20 & 55369.640 & -0.048 & 0.15 & -0.3 & -17.565 & $-1 \cdot 7 E-3$ \\
\hline 19 & 2 & 18 & 20 & 1 & 19 & 56255.000 & 0.002 & 0.10 & 0.0 & -31.280 & $3.9 E-4$ \\
\hline 19 & 3 & 17 & 20 & 2 & 18 & 49445.770 & 0.229 & 0.10 & 1.9 & -69.943 & $-8.0 E-3$ \\
\hline 19 & 4 & 15 & 20 & 4 & 16 & 60361.370 & 0.052 & 0.15 & 0.3 & -34.592 & 0.012 \\
\hline 19 & 5 & 15 & 20 & 5 & 16 & 59679.340 & -0.019 & 0.10 & -0.2 & -25.758 & 0.020 \\
\hline 19 & 5 & 14 & 20 & 5 & 15 & 59746.440 & -0.017 & 0.10 & -0.1 & -26.684 & 0.019 \\
\hline 19 & 6 & 14 & 20 & 6 & 15 & 59554.290 & 0.095 & 0.10 & 0.8 & -21.404 & 0.028 \\
\hline 19 & 6 & 13 & 20 & 6 & 14 & 59557.750 & -0.158 & 0.10 & $-1 \cdot 3$ & -21.468 & 0.028 \\
\hline 19 & 7 & 13 & 20 & 7 & 14 & 59469.270 & 0.136 & 0.15 & 0.8 & -16.442 & 0.038 \\
\hline 19 & 7 & 12 & 20 & 7 & 13 & 59469.270 & 0.006 & 0.15 & 0.0 & -16.445 & 0.038 \\
\hline 19 & 8 & 12 & 20 & 8 & 13 & 59416.650 & 0.037 & 0.10 & 0.3 & -10.988 & 0.050 \\
\hline 19 & 8 & 11 & 20 & 8 & 12 & 59416.650 & 0.034 & 0.10 & 0.3 & -10.988 & 0.050 \\
\hline 19 & 9 & 11 & 20 & 9 & 12 & 59383.450 & 0.036 & 0.10 & 0.3 & -4.969 & 0.064 \\
\hline 19 & 9 & 10 & 20 & 9 & 11 & 59383.450 & 0.036 & 0.10 & 0.3 & -4.969 & 0.064 \\
\hline 19 & 10 & 10 & 20 & 10 & 11 & 59362.160 & -0.076 & 0.10 & -0.6 & 1.659 & 0.079 \\
\hline 19 & 10 & 9 & 20 & 10 & 10 & 59362.160 & -0.076 & 0.10 & -0.6 & 1.659 & 0.079 \\
\hline 19 & 11 & 9 & 20 & 11 & 10 & 59348.980 & -0.066 & 0.10 & -0.6 & 8.922 & 0.095 \\
\hline
\end{tabular}




\begin{tabular}{|c|c|c|c|c|c|c|c|c|c|c|c|}
\hline 19 & 11 & 8 & 20 & 11 & 9 & 59348.980 & -0.066 & 0.10 & -0.6 & 8.922 & 0.095 \\
\hline 19 & 16 & 4 & 20 & 16 & 5 & 59343.980 & -0.303 & 0.15 & $-1 \cdot 7$ & 55.125 & 0.201 \\
\hline 19 & 16 & 3 & 20 & 16 & 4 & 59343.980 & -0.303 & 0.15 & -1.7 & 55.125 & 0.201 \\
\hline 20 & 1 & 20 & 20 & 2 & 19 & 41185.540 & -0.003 & 0.20 & -0.0 & -25.475 & 0.023 \\
\hline 20 & 4 & 17 & 20 & 5 & 16 & 46372.870 & -0.304 & 0.10 & -2.6 & 19.985 & 0.038 \\
\hline 20 & 5 & 15 & 20 & 6 & 14 & 56649.400 & -0.172 & 0.15 & $-1 \cdot 0$ & 22.644 & 0.048 \\
\hline 20 & 0 & 20 & 21 & 1 & 21 & 58194.200 & 0.091 & 0.10 & 0.8 & -19.097 & $-2.0 E-3$ \\
\hline 20 & 1 & 20 & 21 & 0 & 21 & 58101.040 & -0.055 & 0.10 & -0.5 & -20.058 & $-2 \cdot 3 E-3$ \\
\hline 20 & 5 & 16 & 21 & 5 & 17 & 62700.520 & -0.110 & 0.12 & -0.8 & -30.915 & 0.023 \\
\hline 20 & 5 & 15 & 21 & 5 & 16 & 62803.220 & 0.228 & 0.13 & 1.5 & -32.448 & 0.022 \\
\hline 20 & 6 & 15 & 21 & 6 & 16 & 62564.050 & 0.256 & 0.10 & 2.1 & -26.324 & 0.033 \\
\hline 20 & 7 & 14 & 21 & 7 & 15 & 62465.370 & 0.179 & 0.15 & 1.0 & -20.999 & 0.044 \\
\hline 20 & 7 & 13 & 21 & 7 & 14 & 62465.370 & -0.071 & 0.15 & -0.4 & -21.004 & 0.044 \\
\hline 20 & 8 & 13 & 21 & 8 & 14 & 62403.730 & 0.015 & 0.10 & 0.1 & -15.188 & 0.058 \\
\hline 20 & 8 & 12 & 21 & 8 & 13 & 62403.730 & 0.009 & 0.10 & 0.1 & -15.188 & 0.058 \\
\hline 20 & 9 & 12 & 21 & 9 & 13 & 62364.590 & -0.019 & 0.10 & -0.2 & -8.812 & 0.074 \\
\hline 20 & 9 & 11 & 21 & 9 & 12 & 62364.590 & -0.020 & 0.10 & -0.2 & -8.812 & 0.074 \\
\hline 20 & 10 & 11 & 21 & 10 & 12 & 62339.360 & -0.025 & 0.10 & -0.2 & -1.813 & 0.091 \\
\hline 20 & 10 & 10 & 21 & 10 & 11 & 62339.360 & -0.025 & 0.10 & -0.2 & -1.813 & 0.091 \\
\hline 20 & 12 & 9 & 21 & 12 & 10 & 62313.520 & -0.215 & 0.10 & $-1 \cdot 8$ & 14.175 & 0.131 \\
\hline 20 & 12 & 8 & 21 & 12 & 9 & 62313.520 & -0.215 & 0.10 & -1.8 & 14.175 & 0.131 \\
\hline 21 & 4 & 17 & 21 & 5 & 16 & 43682.250 & -0.186 & 0.10 & -1.6 & 45.773 & 0.049 \\
\hline 21 & 5 & 17 & 21 & 6 & 16 & 56676.320 & -0.028 & 0.10 & -0.2 & 25.258 & 0.058 \\
\hline 21 & 5 & 16 & 21 & 6 & 15 & 56416.770 & 0.038 & 0.10 & 0.3 & 28.651 & 0.058 \\
\hline 21 & 6 & 15 & 21 & 7 & 14 & 67446.200 & 0.314 & 0.15 & 1.8 & 18.725 & 0.068 \\
\hline 21 & 0 & 21 & 22 & 1 & 22 & 60891.740 & 0.051 & 0.10 & 0.4 & -22.042 & $-2 \cdot 8 E-3$ \\
\hline 21 & 2 & 20 & 22 & 1 & 21 & 62324.840 & 0.029 & 0.15 & 0.2 & -35.219 & 1. $7 E-3$ \\
\hline 21 & 4 & 17 & 22 & 4 & 18 & 66772.070 & -0.181 & 0.20 & -0.8 & -50.075 & 0.015 \\
\hline 21 & 5 & 17 & 22 & 5 & 18 & 65723.770 & 0.009 & 0.10 & 0.1 & -36.626 & 0.026 \\
\hline 21 & 5 & 16 & 22 & 5 & 17 & 65875.960 & -0.002 & 0.10 & -0.0 & -39.085 & 0.026 \\
\hline 21 & 6 & 16 & 22 & 6 & 17 & 65577.840 & 0.051 & 0.10 & 0.4 & -31.849 & 0.037 \\
\hline 21 & 6 & 15 & 22 & 6 & 16 & 65588.340 & -0.027 & 0.10 & -0.2 & -32.062 & 0.037 \\
\hline 21 & 8 & 14 & 22 & 8 & 15 & 65393.300 & 0.079 & 0.10 & 0.7 & -19.951 & 0.067 \\
\hline 21 & 8 & 13 & 22 & 8 & 14 & 65393.300 & 0.065 & 0.10 & 0.5 & -19.952 & 0.067 \\
\hline 21 & 9 & 13 & 22 & 9 & 14 & 65347.640 & 0.073 & 0.10 & 0.6 & -13.204 & 0.085 \\
\hline 21 & 9 & 12 & 22 & 9 & 13 & 65347.640 & 0.073 & 0.10 & 0.6 & -13.204 & 0.085 \\
\hline 21 & 10 & 12 & 22 & 10 & 13 & 65317.820 & -0.031 & 0.10 & -0.3 & -5.822 & 0.105 \\
\hline 21 & 10 & 11 & 22 & 10 & 12 & 65317.820 & -0.031 & 0.10 & -0.3 & -5.822 & 0.105 \\
\hline 21 & 11 & 11 & 22 & 11 & 12 & 65298.570 & -0.073 & 0.10 & -0.6 & 2.234 & 0.127 \\
\hline 21 & 11 & 10 & 22 & 11 & 11 & 65298.570 & -0.073 & 0.10 & -0.6 & 2.234 & 0.127 \\
\hline 21 & 12 & 10 & 22 & 12 & 11 & 65286.600 & -0.157 & 0.12 & -1.1 & 10.990 & 0.151 \\
\hline 21 & 12 & 9 & 22 & 12 & 10 & 65286.600 & -0.157 & 0.12 & -1.1 & 10.990 & 0.151 \\
\hline 21 & 13 & 9 & 22 & 13 & 10 & 65280.170 & -0.046 & 0.10 & -0.4 & 20.461 & 0.177 \\
\hline 21 & 13 & 8 & 22 & 13 & 9 & 65280.170 & -0.046 & 0.10 & -0.4 & 20.461 & 0.177 \\
\hline 22 & 2 & 21 & 22 & 3 & 20 & 40532.600 & -0.226 & 0.10 & $-1 \cdot 9$ & -30.337 & 0.034 \\
\hline 22 & 3 & 20 & 22 & 4 & 19 & 40145.500 & 0.089 & 0.15 & 0.5 & -3.558 & 0.041 \\
\hline 22 & 4 & 19 & 22 & 5 & 18 & 46431.750 & -0.175 & 0.10 & -1.5 & 23.136 & 0.055 \\
\hline 22 & 4 & 18 & 22 & 5 & 17 & 42785.980 & -0.167 & 0.20 & -0.7 & 56.763 & 0.059 \\
\hline 22 & 5 & 17 & 22 & 6 & 16 & 56129.380 & 0.244 & 0.10 & 2.1 & 35.674 & 0.070 \\
\hline 22 & 0 & 22 & 23 & 1 & 23 & 63593.340 & 0.127 & 0.10 & 1.1 & -25.238 & $-3.8 E-3$ \\
\hline 22 & 1 & 22 & 23 & 0 & 23 & 63547.720 & -0.140 & 0.10 & $-1 \cdot 2$ & -25.861 & $-4.0 E-3$ \\
\hline 22 & 2 & 21 & 23 & 1 & 22 & 65253.720 & 0.047 & 0.10 & 0.4 & -37.543 & $2.5 E-3$ \\
\hline 22 & 5 & 17 & 23 & 5 & 18 & 68968.390 & 0.047 & 0.10 & 0.4 & -46.719 & 0.029 \\
\hline 22 & 6 & 17 & 23 & 6 & 18 & 68596.240 & 0.055 & 0.10 & 0.5 & -38.014 & 0.043 \\
\hline 22 & 6 & 16 & 23 & 6 & 17 & 68613.380 & 0.053 & 0.10 & 0.4 & -38.387 & 0.043 \\
\hline 22 & 8 & 15 & 23 & 8 & 16 & 68385.400 & 0.141 & 0.10 & 1.2 & -25.311 & 0.076 \\
\hline 22 & 8 & 14 & 23 & 8 & 15 & 68385.400 & 0.114 & 0.10 & 0.9 & $-25 \cdot 312$ & 0.076 \\
\hline 22 & 9 & 14 & 23 & 9 & 15 & 68332.420 & 0.045 & 0.10 & 0.4 & -18.174 & 0.097 \\
\hline 22 & 9 & 13 & 23 & 9 & 14 & 68332.420 & 0.044 & 0.10 & 0.4 & -18.174 & 0.097 \\
\hline 22 & 10 & 13 & 23 & 10 & 14 & 68297.770 & 0.073 & 0.10 & 0.6 & -10.399 & 0.119 \\
\hline 22 & 10 & 12 & 23 & 10 & 13 & 68297.770 & 0.073 & 0.10 & 0.6 & -10.399 & 0.119 \\
\hline 22 & 11 & 12 & 23 & 11 & 13 & 68274.930 & -0.050 & 0.10 & -0.4 & -1.933 & 0.145 \\
\hline
\end{tabular}




\begin{tabular}{|c|c|c|c|c|c|c|c|c|c|c|c|}
\hline 22 & 11 & 11 & 23 & 11 & 12 & 68274.930 & -0.050 & 0.10 & -0.4 & -1.933 & 0.145 \\
\hline 22 & 12 & 11 & 23 & 12 & 12 & 68260.520 & -0.046 & 0.10 & -0.4 & 7.254 & 0.172 \\
\hline 22 & 12 & 10 & 23 & 12 & 11 & 68260.520 & -0.046 & 0.10 & -0.4 & 7.254 & 0.172 \\
\hline 22 & 13 & 10 & 23 & 13 & 11 & 68252.140 & -0.049 & 0.10 & -0.4 & 17.181 & 0.202 \\
\hline 22 & 13 & 9 & 23 & 13 & 10 & 68252.140 & -0.049 & 0.10 & -0.4 & 17.181 & 0.202 \\
\hline 23 & 2 & 22 & 23 & 3 & 21 & 42398.370 & -0.183 & 0.10 & -1.5 & -37.452 & 0.041 \\
\hline 23 & 3 & 21 & 23 & 4 & 20 & 41099.450 & 0.187 & 0.10 & 1.6 & -9.172 & 0.048 \\
\hline 23 & 4 & 20 & 23 & 5 & 19 & 46547.270 & -0.160 & 0.10 & $-1 \cdot 3$ & 23.556 & 0.065 \\
\hline 23 & 4 & 19 & 23 & 5 & 18 & 41747.680 & -0.147 & 0.10 & $-1 \cdot 2$ & 69.011 & 0.071 \\
\hline 23 & 5 & 18 & 23 & 6 & 17 & 55774.540 & 0.419 & 0.15 & 2.3 & 44.007 & 0.084 \\
\hline 23 & 0 & 23 & 24 & 1 & 24 & 66297.590 & 0.121 & 0.10 & 1.0 & -28.698 & $-4 \cdot 9 E-3$ \\
\hline 23 & 1 & 23 & 24 & 0 & 24 & 66265.870 & -0.088 & 0.10 & -0.7 & -29.192 & $-5.1 E-3$ \\
\hline 23 & 1 & 22 & 24 & 2 & 23 & 69100.150 & 0.015 & 0.10 & 0.1 & -27.326 & $8.1 E-3$ \\
\hline 23 & 2 & 22 & 24 & 1 & 23 & 68129.690 & -0.042 & 0.10 & -0.4 & -40.199 & $3.3 E-3$ \\
\hline 23 & 5 & 18 & 24 & 5 & 19 & 72083.400 & 0.096 & 0.10 & 0.8 & -55.485 & 0.033 \\
\hline 23 & 6 & 18 & 24 & 6 & 19 & 71618.900 & 0.016 & 0.10 & 0.1 & -44.851 & 0.049 \\
\hline 23 & 6 & 17 & 24 & 6 & 18 & 71646.030 & 0.034 & 0.10 & 0.3 & -45.486 & 0.048 \\
\hline 23 & 8 & 16 & 24 & 8 & 17 & 71379.990 & 0.034 & 0.10 & 0.3 & -31.303 & 0.087 \\
\hline 23 & 8 & 15 & 24 & 8 & 16 & 71379.990 & -0.019 & 0.10 & -0.2 & -31.304 & 0.087 \\
\hline 23 & 9 & 15 & 24 & 9 & 16 & 71319.120 & -0.002 & 0.10 & -0.0 & -23.757 & 0.110 \\
\hline 23 & 9 & 14 & 24 & 9 & 15 & 71319.120 & -0.004 & 0.10 & -0.0 & -23.757 & 0.110 \\
\hline 23 & 10 & 14 & 24 & 10 & 15 & 71279.020 & 0.032 & 0.10 & 0.3 & -15.575 & 0.136 \\
\hline 23 & 10 & 13 & 24 & 10 & 14 & 71279.020 & 0.032 & 0.10 & 0.3 & -15.575 & 0.136 \\
\hline 23 & 11 & 13 & 24 & 11 & 14 & 71252.290 & -0.114 & 0.10 & -1.0 & -6.689 & 0.164 \\
\hline 23 & 11 & 12 & 24 & 11 & 13 & 71252.290 & -0.114 & 0.10 & -1.0 & -6.689 & 0.164 \\
\hline 23 & 12 & 12 & 24 & 12 & 13 & 71235.150 & -0.045 & 0.10 & -0.4 & 2.936 & 0.195 \\
\hline 23 & 12 & 11 & 24 & 12 & 12 & 71235.150 & -0.045 & 0.10 & -0.4 & 2.936 & 0.195 \\
\hline 23 & 13 & 11 & 24 & 13 & 12 & 71224.650 & -0.128 & 0.15 & -0.7 & 13.326 & 0.229 \\
\hline 23 & 13 & 10 & 24 & 13 & 11 & 71224.650 & -0.128 & 0.15 & -0.7 & 13.326 & 0.229 \\
\hline 24 & 1 & 23 & 24 & 2 & 22 & 40152.560 & 0.045 & 0.10 & 0.4 & -86.890 & 0.032 \\
\hline 24 & 2 & 23 & 24 & 3 & 22 & 44348.640 & 0.045 & 0.10 & 0.4 & -45.217 & 0.050 \\
\hline 24 & 3 & 22 & 24 & 4 & 21 & 42187.790 & 0.060 & 0.10 & 0.5 & -16.161 & 0.057 \\
\hline 24 & 4 & 21 & 24 & 5 & 20 & 46737.880 & 0.021 & 0.10 & 0.2 & 22.882 & 0.076 \\
\hline 24 & 4 & 20 & 24 & 5 & 19 & 40583.000 & -0.187 & 0.10 & -1.6 & 81.926 & 0.084 \\
\hline 24 & 5 & 19 & 24 & 6 & 18 & 55336.720 & -0.092 & 0.10 & -0.8 & 54.005 & 0.100 \\
\hline 24 & 6 & 18 & 24 & 7 & 17 & 67007.480 & 0.143 & 0.10 & 1.2 & 38.224 & 0.116 \\
\hline 24 & 0 & 24 & 25 & 1 & 25 & 69003.590 & 0.013 & 0.10 & 0.1 & -32.435 & $-6 \cdot 3 E-3$ \\
\hline 24 & 1 & 24 & 25 & 0 & 25 & 68981.620 & -0.127 & 0.10 & -1.1 & -32.823 & $-6.5 E-3$ \\
\hline 24 & 2 & 23 & 25 & 1 & 24 & 70964.340 & 0.083 & 0.10 & 0.7 & -43.234 & $4.1 E-3$ \\
\hline 24 & 3 & 22 & 25 & 2 & 23 & 69605.030 & 0.139 & 0.10 & 1.2 & -91.908 & $-3.7 E-3$ \\
\hline 24 & 5 & 19 & 25 & 5 & 20 & 75224.240 & 0.339 & 0.10 & 2.8 & -65.514 & 0.036 \\
\hline 24 & 6 & 19 & 25 & 6 & 20 & 74645.660 & 0.000 & 0.10 & 0.0 & -52.387 & 0.055 \\
\hline 24 & 6 & 18 & 25 & 6 & 19 & 74687.630 & 0.054 & 0.10 & 0.5 & -53.440 & 0.055 \\
\hline 24 & 7 & 18 & 25 & 7 & 19 & 74484.920 & 0.188 & 0.10 & 1.6 & -45.441 & 0.075 \\
\hline 24 & 7 & 17 & 25 & 7 & 18 & 74487.400 & 0.202 & 0.10 & 1.7 & -45.515 & 0.075 \\
\hline 24 & 9 & 16 & 25 & 9 & 17 & 74307.960 & 0.060 & 0.10 & 0.5 & -29.985 & 0.124 \\
\hline 24 & 9 & 15 & 25 & 9 & 16 & 74307.960 & 0.057 & 0.10 & 0.5 & -29.985 & 0.124 \\
\hline 24 & 11 & 14 & 25 & 11 & 15 & 74230.840 & -0.122 & 0.10 & -1.0 & -12.063 & 0.186 \\
\hline 24 & 11 & 13 & 25 & 11 & 14 & 74230.840 & -0.122 & 0.10 & -1.0 & -12.063 & 0.186 \\
\hline 25 & 1 & 24 & 25 & 2 & 23 & 42988.960 & -0.269 & 0.10 & $-2 \cdot 3$ & -93.891 & 0.042 \\
\hline 25 & 3 & 23 & 25 & 4 & 22 & 43410.700 & 0.020 & 0.10 & 0.2 & -24.572 & 0.066 \\
\hline 25 & 5 & 20 & 25 & 6 & 19 & 54800.320 & -0.167 & 0.10 & -1.4 & 66.079 & 0.118 \\
\hline 25 & 0 & 25 & 26 & 1 & 26 & 71711.050 & 0.149 & 0.10 & 1.3 & -36.462 & $-8.0 E-3$ \\
\hline 25 & 1 & 25 & 26 & 0 & 26 & 71695.820 & 0.003 & 0.10 & 0.0 & -36.765 & $-8.2 E-3$ \\
\hline 25 & 1 & 24 & 26 & 2 & 25 & 74287.240 & 0.020 & 0.10 & 0.2 & -37.616 & $8.8 E-3$ \\
\hline 25 & 2 & 24 & 26 & 1 & 25 & 73766.520 & -0.090 & 0.10 & -0.8 & -46.682 & 4. $9 E-3$ \\
\hline 25 & 5 & 21 & 26 & 5 & 22 & 77806.090 & -0.088 & 0.10 & -0.7 & -64.921 & 0.043 \\
\hline 25 & 5 & 20 & 26 & 5 & 21 & 78392.770 & 0.197 & 0.10 & 1.7 & -76.914 & 0.040 \\
\hline 25 & 6 & 20 & 26 & 6 & 21 & 77676.020 & -0.109 & 0.10 & -0.9 & -60.646 & 0.062 \\
\hline 25 & 6 & 19 & 26 & 6 & 20 & 77739.620 & 0.061 & 0.10 & 0.5 & -62.349 & 0.061 \\
\hline 25 & 7 & 19 & 26 & 7 & 20 & 77499.380 & 0.140 & 0.10 & 1.2 & -53.297 & 0.084 \\
\hline 25 & 7 & 18 & 26 & 7 & 19 & 77503.250 & -0.085 & 0.10 & -0.7 & -53.429 & 0.084 \\
\hline
\end{tabular}




\begin{tabular}{|c|c|c|c|c|c|c|c|c|c|c|}
\hline 25 & 18 & 26 & 8 & 19 & 77377.950 & 0.086 & 0.12 & 0.6 & -45.326 & 0.110 \\
\hline 25 & 17 & 26 & 8 & 18 & 77377.950 & -0.094 & 0.12 & -0.7 & -45.333 & 0.110 \\
\hline 25 & 17 & 26 & 9 & 18 & 77298.740 & -0.060 & 0.10 & -0.5 & -36.892 & 0.140 \\
\hline 25 & 16 & 26 & 9 & 17 & 77298.740 & -0.066 & 0.10 & -0.6 & -36.892 & 0.140 \\
\hline 2510 & 16 & 26 & 10 & 17 & 77246.150 & -0.008 & 0.10 & -0.1 & -27.845 & 0.172 \\
\hline $25 \quad 10$ & 15 & 26 & 10 & 16 & 77246.150 & -0.009 & 0.10 & -0.1 & -27.845 & 0.172 \\
\hline 11 & 15 & 26 & 11 & 16 & 77210.490 & -0.212 & 0.15 & -1.2 & -18.086 & 0.209 \\
\hline 2511 & 14 & 26 & 11 & 15 & 77210.490 & -0.212 & 0.15 & -1.2 & -18.086 & 0.209 \\
\hline $25 \quad 12$ & 14 & 26 & 12 & 15 & 77186.950 & -0.103 & 0.10 & -0.9 & -7.555 & 0.248 \\
\hline $25 \quad 12$ & 13 & 26 & 12 & 14 & 77186.950 & -0.103 & 0.10 & -0.9 & -7.555 & 0.248 \\
\hline $25 \quad 13$ & 13 & 26 & 13 & 14 & 77171.630 & -0.274 & 0.10 & $-2 \cdot 3$ & 3.781 & 0.292 \\
\hline 13 & 12 & 26 & 13 & 13 & 77171.630 & -0.274 & 0.10 & $-2 \cdot 3$ & 3.781 & 0.292 \\
\hline 1 & 25 & 26 & 2 & 24 & 45770.870 & -0.047 & 0.10 & -0.4 & -100.356 & 0.054 \\
\hline 3 & 24 & 26 & 4 & 23 & 44765.670 & -0.001 & 0.10 & -0.0 & -34.412 & 0.077 \\
\hline 4 & 23 & 26 & 5 & 22 & 47394.590 & -0.096 & 0.10 & -0.8 & 17.234 & 0.102 \\
\hline 5 & 22 & 26 & 6 & 21 & 55952.750 & 0.059 & 0.10 & 0.5 & 48.726 & 0.132 \\
\hline 26 & 21 & 26 & 6 & 20 & 54147.390 & -0.083 & 0.10 & -0.7 & 80.644 & 0.139 \\
\hline 26 & 26 & 27 & 1 & 27 & 74419.270 & 0.287 & 0.20 & 1.2 & -40.793 & -0.010 \\
\hline 1 & 26 & 27 & 0 & 27 & 74408.470 & -0.115 & 0.12 & -0.8 & -41.028 & -0.010 \\
\hline 26 & 25 & 27 & 2 & 26 & 76922.370 & -0.023 & 0.10 & -0.2 & -43.095 & $9.1 \mathrm{E}-3$ \\
\hline 26 & 25 & 27 & 1 & 26 & 76544.560 & 0.206 & 0.10 & 1.8 & -50.564 & $5.7 E-3$ \\
\hline 26 & 24 & 27 & 2 & 25 & 76576.090 & 0.264 & 0.10 & 2.2 & -94.645 & $2.8 E-3$ \\
\hline 27 & 26 & 27 & 2 & 25 & 48490.400 & -0.133 & 0.15 & -0.7 & -106.532 & 0.070 \\
\hline 27 & 26 & 27 & 3 & 25 & 50598.870 & -0.082 & 0.10 & -0.7 & -71.797 & 0.087 \\
\hline 27 & 24 & 27 & 5 & 23 & 47884.700 & 0.221 & 0.20 & 0.9 & 11.764 & 0.116 \\
\hline 27 & 23 & 27 & 6 & 22 & 55847.300 & 0.114 & 0.20 & 0.5 & 52.336 & 0.152 \\
\hline 27 & 22 & 27 & 6 & 21 & 53360.610 & -0.187 & 0.10 & $-1 \cdot 6$ & 98.045 & 0.162 \\
\hline 27 & 20 & 27 & 8 & 19 & 77501.740 & 0.347 & 0.10 & 3.0 & 50.747 & 0.211 \\
\hline 27 & 27 & 28 & 1 & 28 & 77127.770 & 0.276 & 0.10 & 2.4 & -45.441 & -0.012 \\
\hline 27 & 27 & 28 & 0 & 28 & 77120.110 & -0.232 & 0.10 & -2.0 & -45.622 & -0.013 \\
\hline 27 & 26 & 28 & 2 & 27 & 79576.730 & 0.112 & 0.10 & 1.0 & -48.804 & $9.3 E-3$ \\
\hline 27 & 26 & 28 & 1 & 27 & 79303.300 & -0.163 & 0.10 & $-1 \cdot 4$ & -54.893 & $6.4 \mathrm{E}-3$ \\
\hline 27 & 25 & 28 & 2 & 26 & 79852.220 & -0.117 & 0.10 & -1.0 & -95.777 & $6.9 E-3$ \\
\hline 28 & 27 & 28 & 2 & 26 & 51147.820 & -0.006 & 0.10 & -0.0 & -112.681 & 0.088 \\
\hline 28 & 27 & 28 & 3 & 26 & 52782.640 & -0.023 & 0.10 & -0.2 & -81.557 & 0.104 \\
\hline 28 & 26 & 28 & 4 & 25 & 47851.530 & 0.071 & 0.20 & 0.3 & -58.206 & 0.104 \\
\hline 28 & 25 & 28 & 5 & 24 & .230 & 0.092 & 0.12 & 0.6 & 4.238 & 0.132 \\
\hline 28 & 24 & 28 & 6 & 23 & 55777.350 & 0.090 & 0.10 & 0.8 & 54.969 & 0.174 \\
\hline 28 & 23 & 28 & 6 & 22 & 52426.620 & -0.038 & 0.10 & -0.3 & 118.429 & 0.189 \\
\hline 29 & 28 & 29 & 3 & 27 & 55001.770 & -0.055 & 0.10 & -0.5 & -91.691 & 0.124 \\
\hline 29 & 27 & 29 & 3 & 26 & 41318.650 & -0.120 & 0.10 & -1.0 & -182.307 & 0.049 \\
\hline 29 & 27 & 29 & 4 & 26 & 49567.470 & -0.127 & 0.10 & -1.1 & -71.986 & 0.120 \\
\hline 29 & 26 & 29 & 5 & 25 & 49234.390 & 0.043 & 0.10 & 0.4 & -5.521 & 0.149 \\
\hline 29 & 24 & 29 & 6 & 23 & 51337.420 & -0.182 & 0.10 & -1.5 & 141.603 & 0.219 \\
\hline 30 & 29 & 30 & 2 & 28 & 56294.180 & -0.175 & 0.15 & -1.0 & -125.796 & 0.133 \\
\hline 30 & 28 & 30 & 3 & 27 & 44411.340 & -0.129 & 0.10 & $-1 \cdot 1$ & -199.611 & 0.064 \\
\hline 30 & 28 & 30 & 4 & 27 & 51387.110 & -0.019 & 0.10 & -0.2 & -86.856 & 0.138 \\
\hline 30 & 27 & 30 & 5 & 26 & 50107.890 & 0.232 & 0.20 & 1.0 & -17.637 & 0.168 \\
\hline 30 & 26 & 30 & 6 & 25 & 55793.230 & -0.050 & 0.10 & -0.4 & 55.863 & 0.224 \\
\hline 30 & 25 & 30 & 6 & 24 & 50096.040 & 0.053 & 0.10 & 0.5 & 166.876 & 0.253 \\
\hline 30 & 24 & 30 & 7 & 23 & 65096.670 & -0.078 & 0.10 & -0.7 & 107.065 & 0.283 \\
\hline 30 & 24 & 30 & 8 & 23 & 77007.630 & -0.145 & 0.12 & -1.0 & 79.197 & 0.320 \\
\hline 31 & 30 & 31 & 2 & 29 & 58797.620 & 0.209 & 0.10 & 1.8 & -133.091 & 0.161 \\
\hline 31 & 29 & 31 & 3 & 28 & 47496.230 & -0.002 & 0.10 & -0.0 & -214.361 & 0.084 \\
\hline 31 & 29 & 31 & 4 & 28 & 53299.820 & 0.012 & 0.10 & 0.1 & -102.670 & 0.160 \\
\hline 31 & 26 & 31 & 6 & 25 & 48716.930 & -0.081 & 0.10 & -0.7 & 192.960 & 0.288 \\
\hline 31 & 26 & 31 & 7 & 25 & 65679.520 & 0.004 & 0.10 & 0.0 & 91.151 & 0.313 \\
\hline 31 & 25 & 31 & 7 & 24 & 64536.570 & 0.032 & 0.15 & 0.2 & 126.534 & 0.325 \\
\hline 32 & 30 & 32 & 3 & 29 & 50539.520 & -0.040 & 0.10 & -0.3 & -226.739 & 0.109 \\
\hline 32 & 30 & 32 & 4 & 29 & 55294.800 & -0.131 & 0.10 & -1.1 & -119.276 & 0.184 \\
\hline 32 & 29 & 32 & 5 & 28 & 52267.440 & 0.218 & 0.10 & 1.8 & -49.146 & 0.211 \\
\hline 32 & 28 & 32 & 6 & 27 & 56103.950 & 0.029 & 0.10 & 0.2 & 48.305 & 0.281 \\
\hline
\end{tabular}




\begin{tabular}{|c|c|c|c|c|c|c|c|c|c|c|}
\hline 32 & 527 & 32 & 6 & 26 & 47230.640 & 0.099 & 0.10 & 0.8 & 217.957 & 0.326 \\
\hline 32 & 626 & 32 & 7 & 25 & 63869.040 & 0.027 & 0.10 & 0.2 & 149.843 & 0.372 \\
\hline 32 & 725 & 32 & 8 & 24 & 76426.720 & 0.064 & 0.10 & 0.6 & 107.847 & 0.415 \\
\hline 33 & 232 & 33 & 3 & 31 & 64100.950 & -0.167 & 0.10 & $-1 \cdot 4$ & -135.611 & 0.239 \\
\hline 33 & 529 & 33 & 6 & 28 & 56402.000 & 0.133 & 0.15 & 0.7 & 40.375 & 0.312 \\
\hline 33 & 627 & 33 & 7 & 26 & 63076.720 & 0.087 & 0.10 & 0.7 & 177.642 & 0.424 \\
\hline 33 & 727 & 33 & 8 & 26 & 76338.800 & -0.169 & 0.10 & -1.4 & 113.506 & 0.466 \\
\hline 33 & 726 & 33 & 8 & 25 & 76110.770 & 0.241 & 0.10 & 2.1 & 123.457 & 0.470 \\
\hline 34 & 233 & 34 & 3 & 32 & 66407.120 & -0.075 & 0.10 & -0.6 & -147.450 & 0.279 \\
\hline 34 & 232 & 34 & 3 & 31 & 56424.050 & 0.032 & 0.10 & 0.3 & -246.395 & 0.175 \\
\hline 34 & 332 & 34 & 4 & 31 & 59489.720 & 0.046 & 0.10 & 0.4 & -154.284 & 0.245 \\
\hline 34 & 331 & 34 & 4 & 30 & 41363.360 & -0.273 & 0.15 & -1.5 & -302.895 & 0.064 \\
\hline 34 & 31 & 34 & 5 & 30 & 54971.590 & -0.071 & 0.10 & -0.6 & -90.041 & 0.262 \\
\hline 34 & 629 & 34 & 7 & 28 & 65082.050 & -0.059 & 0.10 & -0.5 & 110.306 & 0.444 \\
\hline 34 & 628 & 34 & 7 & 27 & 62142.520 & -0.001 & 0.10 & -0.0 & 210.421 & 0.483 \\
\hline 34 & 728 & 34 & 8 & 27 & 76082.090 & -0.179 & 0.10 & -1.5 & 125.963 & 0.524 \\
\hline 34 & 727 & 34 & 8 & 26 & 75746.270 & 0.088 & 0.10 & 0.8 & 141.293 & 0.531 \\
\hline 35 & 234 & 35 & 3 & 33 & 68719.040 & 0.033 & 0.10 & 0.3 & -159.666 & 0.324 \\
\hline 35 & 233 & 35 & 3 & 32 & 59250.170 & -0.170 & 0.10 & $-1 \cdot 4$ & -254.944 & 0.217 \\
\hline 35 & 332 & 35 & 4 & 31 & 44481.320 & -0.040 & 0.10 & -0.3 & -345.044 & 0.077 \\
\hline 35 & 432 & 35 & 5 & 31 & 56517.740 & -0.051 & 0.10 & -0.4 & -113.650 & 0.292 \\
\hline 35 & 630 & 35 & 7 & 29 & 64938.070 & -0.057 & 0.10 & -0.5 & 113.664 & 0.494 \\
\hline 35 & 629 & 35 & 7 & 28 & 61052.770 & 0.018 & 0.10 & 0.2 & 248.308 & 0.548 \\
\hline 35 & 728 & 35 & 8 & 27 & 75324.520 & 0.014 & 0.10 & 0.1 & 161.922 & 0.598 \\
\hline 36 & 334 & 36 & 4 & 33 & 63890.050 & -0.001 & 0.10 & -0.0 & -190.898 & 0.324 \\
\hline 36 & 33 & 36 & 5 & 32 & 58183.700 & -0.114 & 0.10 & -1.0 & -139.068 & 0.326 \\
\hline 36 & 532 & 36 & 6 & 31 & 57999.090 & 0.132 & 0.10 & 1.1 & -3.909 & 0.416 \\
\hline 36 & 531 & 36 & 6 & 30 & 41270.580 & 0.044 & 0.10 & 0.4 & 255.384 & 0.461 \\
\hline 36 & 631 & 36 & 7 & 30 & 64840.640 & -0.041 & 0.10 & -0.3 & 114.629 & 0.547 \\
\hline 36 & 730 & 36 & 8 & 29 & 75529.320 & -0.163 & 0.10 & $-1 \cdot 4$ & 151.609 & 0.655 \\
\hline 36 & 729 & 36 & 8 & 28 & 74834.420 & -0.101 & 0.10 & -0.9 & 186.049 & 0.672 \\
\hline 37 & 35 & 37 & 3 & 34 & 64682.840 & 0.103 & 0.10 & 0.9 & -272.370 & 0.319 \\
\hline 37 & 334 & 37 & 4 & 33 & 50995.480 & 0.047 & 0.10 & 0.4 & -414.169 & 0.126 \\
\hline 37 & 434 & 37 & 5 & 33 & 59960.920 & 0.086 & 0.10 & 0.7 & -166.022 & 0.363 \\
\hline 37 & 32 & 37 & 6 & 31 & 40111.930 & -0.233 & 0.10 & -2.0 & 235.899 & 0.481 \\
\hline 37 & 632 & 37 & 7 & 31 & 64802.450 & -0.004 & 0.10 & -0.0 & 112.614 & 0.602 \\
\hline 37 & 731 & 37 & 8 & 30 & 75240.520 & -0.043 & 0.10 & -0.4 & 164.297 & 0.729 \\
\hline 38 & 237 & 38 & 3 & 36 & 75669.440 & -0.098 & 0.10 & -0.9 & -198.865 & 0.497 \\
\hline 38 & 236 & 38 & 3 & 35 & 67303.440 & 0.033 & 0.10 & 0.3 & -282.063 & 0.380 \\
\hline 38 & 36 & 38 & 4 & 35 & 68426.600 & 0.017 & 0.10 & 0.1 & -228.520 & 0.428 \\
\hline 38 & 335 & 38 & 4 & 34 & 54281.770 & -0.046 & 0.10 & -0.4 & -439.832 & 0.166 \\
\hline 38 & 35 & 38 & 5 & 34 & 61838.910 & 0.010 & 0.10 & 0.1 & -194.221 & 0.406 \\
\hline 38 & 633 & 38 & 7 & 32 & 64836.620 & 0.091 & 0.10 & 0.8 & 107.020 & 0.660 \\
\hline 38 & 632 & 38 & 7 & 31 & 56819.000 & 0.045 & 0.10 & 0.4 & 383.557 & 0.781 \\
\hline 38 & 732 & 38 & 8 & 31 & 74949.870 & -0.193 & 0.10 & $-1 \cdot 6$ & 176.419 & 0.807 \\
\hline 38 & 31 & 38 & 8 & 30 & 73595.560 & -0.110 & 0.10 & -0.9 & 248.317 & 0.845 \\
\hline 39 & 36 & 39 & 4 & 35 & 57529.940 & -0.081 & 0.10 & -0.7 & -459.855 & 0.217 \\
\hline 39 & 35 & 39 & 5 & 34 & 41223.270 & -0.164 & 0.10 & $-1 \cdot 4$ & -392.324 & 0.116 \\
\hline 39 & 535 & 39 & 6 & 34 & 60803.040 & 0.243 & 0.10 & 2.0 & -82.360 & 0.538 \\
\hline 39 & 634 & 39 & 7 & 33 & 64956.020 & 0.031 & 0.10 & 0.3 & 97.264 & 0.720 \\
\hline 39 & 33 & 39 & 8 & 32 & 74664.720 & 0.017 & 0.10 & 0.1 & 187.497 & 0.891 \\
\hline 39 & 32 & 39 & 8 & 31 & 72815.120 & -0.091 & 0.10 & -0.8 & 288.456 & 0.947 \\
\hline 40 & 38 & 40 & 3 & 37 & 72395.930 & -0.009 & 0.10 & -0.1 & -304.567 & 0.523 \\
\hline 40 & 337 & 40 & 4 & 36 & 60711.390 & 0.074 & 0.10 & 0.6 & -475.186 & 0.279 \\
\hline 40 & 437 & 40 & 5 & 36 & 65855.480 & 0.043 & 0.10 & 0.4 & -253.195 & 0.507 \\
\hline 40 & 36 & 40 & 6 & 35 & 62019.240 & 0.094 & 0.10 & 0.8 & -115.975 & 0.584 \\
\hline 40 & 35 & 40 & 7 & 34 & 65173.640 & 0.149 & 0.10 & 1.3 & 82.806 & 0.781 \\
\hline 40 & 34 & 40 & 7 & 33 & 53371.060 & -0.082 & 0.20 & -0.3 & 466.414 & 0.949 \\
\hline 40 & 34 & 40 & 8 & 33 & 74392.630 & 0.063 & 0.10 & 0.5 & 196.958 & 0.980 \\
\hline 41 & 239 & 41 & 3 & 38 & 74883.620 & 0.194 & 0.12 & 1.4 & -317.576 & 0.606 \\
\hline 41 & 339 & 41 & 4 & 38 & 75373.000 & 0.143 & 0.10 & 1.2 & -286.546 & 0.637 \\
\hline 41 & 338 & 41 & 4 & 37 & 63808.470 & -0.086 & 0.10 & -0.7 & -487.101 & 0.353 \\
\hline
\end{tabular}




\begin{tabular}{|c|c|c|c|c|c|c|c|c|c|c|}
\hline 41 & 636 & 41 & 7 & 35 & 65500.970 & 0.133 & 0.10 & 1.1 & 63.178 & 0.845 \\
\hline 41 & 635 & 41 & 7 & 34 & 51587.950 & -0.028 & 0.10 & -0.2 & 493.642 & 1.028 \\
\hline 41 & 734 & 41 & 8 & 33 & 70846.050 & -0.071 & 0.10 & -0.6 & 391.340 & 1.184 \\
\hline 42 & 240 & 42 & 3 & 39 & 77340.570 & -0.018 & 0.10 & -0.2 & -331.794 & 0.698 \\
\hline 42 & 340 & 42 & 4 & 39 & 77707.750 & 0.133 & 0.10 & 1.2 & -306.406 & 0.724 \\
\hline 42 & 339 & 42 & 4 & 38 & 66814.520 & -0.006 & 0.10 & -0.0 & -496.959 & 0.438 \\
\hline 42 & 39 & 42 & 5 & 38 & 70147.310 & 0.084 & 0.10 & 0.7 & -313.900 & 0.638 \\
\hline 42 & 38 & 42 & 5 & 37 & 50249.780 & -0.122 & 0.10 & -1.0 & -636.004 & 0.116 \\
\hline 42 & 538 & 42 & 6 & 37 & 64861.240 & 0.111 & 0.10 & 0.9 & -192.888 & 0.687 \\
\hline 42 & 636 & 42 & 7 & 35 & 49851.730 & -0.073 & 0.10 & -0.6 & 505.149 & 1.096 \\
\hline 42 & 735 & 42 & 8 & 34 & 69626.070 & -0.025 & 0.10 & -0.2 & 454.881 & 1.322 \\
\hline 43 & 340 & 43 & 4 & 39 & 69729.570 & 0.016 & 0.10 & 0.1 & -506.008 & 0.533 \\
\hline 43 & 440 & 43 & 5 & 39 & 72370.820 & 0.089 & 0.10 & 0.8 & -344.410 & 0.716 \\
\hline 43 & 39 & 43 & 5 & 38 & 53629.060 & -0.029 & 0.10 & -0.2 & -702.177 & 0.143 \\
\hline 43 & 539 & 43 & 6 & 38 & 66475.100 & 0.037 & 0.10 & 0.3 & -235.385 & 0.746 \\
\hline 43 & 538 & 43 & 6 & 37 & 40479.700 & 0.030 & 0.10 & 0.2 & -228.440 & 0.385 \\
\hline 43 & 637 & 43 & 7 & 36 & 48236.310 & -0.116 & 0.10 & -1.0 & 496.581 & 1.149 \\
\hline 43 & 737 & 43 & 8 & 36 & 73755.570 & -0.021 & 0.10 & -0.2 & 208.564 & 1.272 \\
\hline 43 & 736 & 43 & 8 & 35 & 68235.410 & 0.184 & 0.10 & 1.6 & 525.715 & 1.473 \\
\hline 44 & 41 & 44 & 4 & 40 & 72559.210 & 0.180 & 0.10 & 1.5 & -515.272 & 0.640 \\
\hline 44 & 41 & 44 & 5 & 40 & 74633.650 & -0.067 & 0.10 & -0.6 & -374.858 & 0.805 \\
\hline 44 & 40 & 44 & 5 & 39 & 57085.550 & -0.089 & 0.10 & -0.8 & -757.032 & 0.189 \\
\hline 44 & 540 & 44 & 6 & 39 & 68207.190 & 0.168 & 0.15 & 0.9 & -279.949 & 0.811 \\
\hline 44 & 539 & 44 & 6 & 38 & 42040.570 & -0.129 & 0.10 & -1.1 & -359.898 & 0.333 \\
\hline 44 & 638 & 44 & 7 & 37 & 46817.020 & 0.001 & 0.10 & 0.0 & 464.326 & 1.183 \\
\hline 45 & 42 & 45 & 4 & 41 & 75311.290 & 0.076 & 0.10 & 0.6 & -525.512 & 0.758 \\
\hline 45 & 42 & 45 & 5 & 41 & 76928.270 & 0.139 & 0.10 & 1.2 & -405.179 & 0.903 \\
\hline 45 & 441 & 45 & 5 & 40 & 60561.340 & -0.221 & 0.10 & -1.9 & -799.961 & 0.256 \\
\hline 45 & 541 & 45 & 6 & 40 & 70047.660 & 0.216 & 0.10 & 1.8 & -326.078 & 0.884 \\
\hline 45 & 640 & 45 & 7 & 39 & 68094.020 & 0.137 & 0.10 & 1.1 & -72.535 & 1.113 \\
\hline 45 & 639 & 45 & 7 & 38 & 45665.400 & 0.051 & 0.10 & 0.4 & 405.770 & 1.195 \\
\hline 45 & 739 & 45 & 8 & 38 & 73598.340 & 0.022 & 0.10 & 0.2 & 194.214 & 1.482 \\
\hline 45 & 738 & 45 & 8 & 37 & 64950.690 & 0.090 & 0.10 & 0.8 & 679.442 & 1.803 \\
\hline 46 & 343 & 46 & 4 & 42 & 77995.780 & 0.205 & 0.10 & 1.8 & -537.236 & 0.886 \\
\hline 46 & 442 & 46 & 5 & 41 & 64008.790 & -0.043 & 0.10 & -0.4 & -831.574 & 0.347 \\
\hline 46 & 42 & 46 & 6 & 41 & 71985.970 & 0.052 & 0.10 & 0.4 & -373.274 & 0.965 \\
\hline 46 & 541 & 46 & 6 & 40 & 46438.210 & -0.205 & 0.10 & -1.7 & -647.162 & 0.220 \\
\hline 46 & 740 & 46 & 8 & 39 & 73638.310 & 0.023 & 0.10 & 0.2 & 177.910 & 1.590 \\
\hline 46 & 739 & 46 & 8 & 38 & 63093.370 & 0.060 & 0.10 & 0.5 & 753.634 & 1.974 \\
\hline 47 & 43 & 47 & 5 & 42 & 67391.380 & -0.090 & 0.10 & -0.8 & -853.493 & 0.460 \\
\hline 47 & 42 & 47 & 6 & 41 & 49204.610 & -0.005 & 0.10 & -0.0 & -793.028 & 0.170 \\
\hline 47 & 641 & 47 & 7 & 40 & 44412.560 & 0.070 & 0.10 & 0.6 & 204.783 & 1.140 \\
\hline 47 & 41 & 47 & 8 & 40 & 73774.620 & 0.202 & 0.10 & 1.7 & 154.510 & 1.698 \\
\hline 47 & 740 & 47 & 8 & 39 & 61141.510 & -0.089 & 0.10 & -0.8 & 817.926 & 2.140 \\
\hline 48 & $4 \quad 44$ & 48 & 5 & 43 & 70686.090 & 0.220 & 0.10 & 1.9 & -867.984 & 0.595 \\
\hline 48 & 44 & 48 & 6 & 43 & 76113.450 & 0.076 & 0.10 & 0.6 & -469.035 & 1.159 \\
\hline 48 & 43 & 48 & 6 & 42 & 52280.270 & -0.070 & 0.10 & -0.6 & -932.742 & 0.135 \\
\hline 48 & 43 & 48 & 7 & 42 & 71521.220 & 0.218 & 0.10 & 1.8 & -233.178 & 1.340 \\
\hline 48 & 642 & 48 & 7 & 41 & 44407.910 & 0.099 & 0.10 & 0.8 & 62.434 & 1.071 \\
\hline 48 & 40 & 48 & 9 & 39 & 79524.200 & -0.191 & 0.10 & -1.7 & 658.687 & 2.537 \\
\hline 49 & 45 & 49 & 5 & 44 & 73879.780 & 0.117 & 0.12 & 0.8 & -877.541 & 0.751 \\
\hline 49 & 44 & 49 & 6 & 43 & 55601.590 & -0.064 & 0.10 & -0.5 & -1060.688 & 0.123 \\
\hline 49 & 44 & 49 & 7 & 43 & 72944.840 & 0.122 & 0.10 & 1.0 & -295.938 & 1.425 \\
\hline 49 & 43 & 49 & 7 & 42 & 44861.660 & 0.109 & 0.10 & 0.9 & -106.171 & 0.975 \\
\hline 49 & 43 & 49 & 8 & 42 & 74382.470 & 0.238 & 0.20 & 1.0 & 84.069 & 1.916 \\
\hline 49 & 42 & 49 & 8 & 41 & 57186.790 & 0.113 & 0.10 & 1.0 & 887.763 & 2.423 \\
\hline 49 & 41 & 49 & 9 & 40 & 78198.500 & -0.211 & 0.10 & -1.8 & 757.412 & 2.798 \\
\hline 50 & 545 & 50 & 6 & 44 & 59099.420 & -0.167 & 0.10 & -1.4 & -1172.156 & 0.142 \\
\hline 50 & 45 & 50 & 7 & 44 & 74500.970 & 0.229 & 0.20 & 1.0 & -362.211 & 1.517 \\
\hline 50 & 644 & 50 & 7 & 43 & 45790.680 & 0.080 & 0.10 & 0.7 & -298.508 & 0.854 \\
\hline 50 & 743 & 50 & 8 & 42 & 55325.450 & 0.115 & 0.10 & 1.0 & 879.096 & 2.521 \\
\hline 50 & 842 & 50 & 9 & 41 & 76696.560 & -0.134 & 0.10 & -1.2 & 867.196 & 3.082 \\
\hline
\end{tabular}




\begin{tabular}{|c|c|c|c|c|c|c|c|c|c|c|}
\hline 51 & 47 & 51 & 5 & 46 & 79959.120 & 0.177 & 0.10 & 1.5 & -890.948 & $1.11 \mathrm{~s}$ \\
\hline 51 & 46 & 51 & 6 & 45 & 62704.810 & -0.254 & 0.10 & -2.2 & -1263.947 & 0.198 \\
\hline 6 & 46 & 51 & 7 & 45 & 76181.680 & 0.300 & 0.10 & 2.5 & -431.229 & 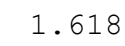 \\
\hline 6 & 45 & 51 & 7 & 44 & 47198.340 & -0.157 & 0.10 & -1.3 & -510.772 & 0.71 \\
\hline 5 & 47 & 52 & 6 & 46 & 66353.780 & -0.014 & 0.10 & -0.1 & -1334.746 & 0.29 \\
\hline 6 & 46 & 52 & 7 & 45 & 49074.870 & 0.080 & 0.10 & 0.7 & -737.650 & 0.5 \\
\hline 7 & 46 & 52 & 8 & 45 & 76273.470 & 0.108 & 0.10 & 0.9 & -85.010 & \\
\hline 7 & 45 & 52 & 8 & 44 & 2220.890 & 0.073 & 0.10 & 0.6 & 746.678 & \\
\hline 5 & 48 & 53 & 6 & 47 & 9990.370 & -0.011 & 0.10 & -0.1 & -1385.214 & \\
\hline 7 & 47 & 53 & 8 & 46 & 7189.810 & 0.278 & 0.10 & 2.3 & -157.994 & \\
\hline 7 & 46 & 53 & 8 & 45 & 1124.490 & 0.074 & 0.10 & 0.6 & 616.620 & \\
\hline 8 & 45 & 53 & 9 & 44 & 1161.170 & -0.132 & 0.10 & -1.2 & 1223.041 & \\
\hline 5 & 49 & 54 & 6 & 48 & 3571.020 & -0.070 & 0.10 & -0.6 & -1417.728 & \\
\hline 6 & 48 & 54 & 7 & 47 & 4117.890 & -0.226 & 0.10 & -1.9 & -1206.405 & \\
\hline 7 & 47 & 54 & 8 & 46 & 0416.230 & 0.103 & 0.10 & 0.9 & 442.786 & \\
\hline 8 & 46 & 54 & 9 & 45 & 45.780 & -0.307 & 0.10 & -2.7 & 6.455 & \\
\hline 6 & 49 & 55 & 7 & 48 & 92.960 & 0.073 & 0.10 & 0.6 & -1431.188 & \\
\hline 7 & 48 & 55 & 8 & 47 & 144.820 & -0.141 & 0.10 & -1.2 & 5.331 & \\
\hline 8 & 47 & 55 & 9 & 46 & 6868.910 & -0.164 & 0.10 & -1.4 & 1405.848 & \\
\hline 7 & 49 & 56 & 8 & 48 & 0347.200 & 0.195 & 0.10 & 1.7 & -30.118 & \\
\hline 6 & 52 & 58 & 7 & 51 & 7858.78 & -0.129 & 0.10 & -1.1 & -1968.869 & \\
\hline 7 & 51 & 58 & 8 & 50 & 247. & 0.101 & 0.10 & 0.9 & -644.905 & \\
\hline 8 & 50 & 58 & 9 & 49 & 100 & -0.044 & 0.10 & -0.4 & 348 & \\
\hline 6 & 53 & 59 & 7 & 52 & 657.650 & -0.099 & 0.10 & -0.9 & -2084.924 & \\
\hline 7 & 52 & 59 & 8 & 51 & 9949.090 & -0.018 & 0.10 & -0.2 & -989.739 & \\
\hline 6 & 54 & 60 & 7 & 53 & 5468.540 & -0.268 & 0.10 & -2.4 & -2167.318 & \\
\hline 8 & 52 & 60 & 9 & 51 & 7565.690 & 0.154 & 0.10 & 1.3 & 1099.417 & \\
\hline 7 & 54 & 61 & 8 & 53 & 753 & -0.169 & 0.10 & -1.5 & -1705.489 & \\
\hline 8 & 53 & 61 & 9 & 52 & 5.020 & 0.122 & 0.10 & 1.0 & .946 & \\
\hline 7 & 55 & 62 & 8 & 54 & 73.150 & 0.106 & 0.10 & 0.9 & -2051.909 & \\
\hline 8 & 54 & 62 & 9 & 53 & 982.770 & 0.061 & 0.10 & 0.5 & 547.211 & \\
\hline 63 & 55 & 63 & 9 & 54 & 5889.380 & 0.087 & 0.10 & 0.7 & 180.166 & \\
\hline 8 & 56 & 64 & 9 & 55 & 6305.290 & 0.055 & 0.10 & 0.5 & -240.150 & \\
\hline 7 & 58 & 65 & 8 & 57 & 2544.200 & -0.203 & 0.10 & -1.9 & -2898.364 & -0 . \\
\hline 8 & 58 & 66 & 9 & 57 & 2.320 & 0.005 & 0.10 & 0.0 & .396 & \\
\hline 67 & 59 & 67 & 9 & 58 & 90.460 & 0.151 & 0.10 & 1.3 & -172 & 1. \\
\hline 67 & 58 & 67 & 10 & 57 & 64242.890 & -0.297 & 0.15 & -1.8 & 1840.488 & 9. \\
\hline 68 & 60 & 68 & 9 & 59 & 63150.950 & 0.151 & 0.10 & 1.3 & -2253.004 & 0.7 \\
\hline 69 & 61 & 69 & 9 & 60 & 66050.030 & 0.082 & 0.10 & 0.8 & -2767.385 & \\
\hline 9 & 60 & 69 & 10 & 59 & 61999.220 & -0.249 & 0.20 & -1.1 & 1108.819 & \\
\hline 9 & 61 & 70 & 10 & 60 & .860 & 0.049 & 0.10 & 0.5 & 611.545 & 7. \\
\hline 8 & 64 & 72 & 9 & 63 & 76758.560 & -0.270 & 0.20 & -1.2 & -4062.706 & -1 \\
\hline 73 & 64 & 73 & 10 & 63 & 63433.100 & 0.241 & 0.15 & 1.4 & -1300.080 & 4 \\
\hline 75 & 66 & 75 & 10 & 65 & 67361.820 & 0.101 & 0.10 & 1.2 & -2776.515 & \\
\hline 10 & 69 & 79 & 11 & 68 & 67405.620 & -0.182 & 0.10 & -2.2 & -220.099 & 10. \\
\hline 10 & 70 & 80 & 11 & 69 & 68200.800 & -0.074 & 0.12 & -0.6 & -1148.331 & \\
\hline
\end{tabular}

rms deviation: 1.2022

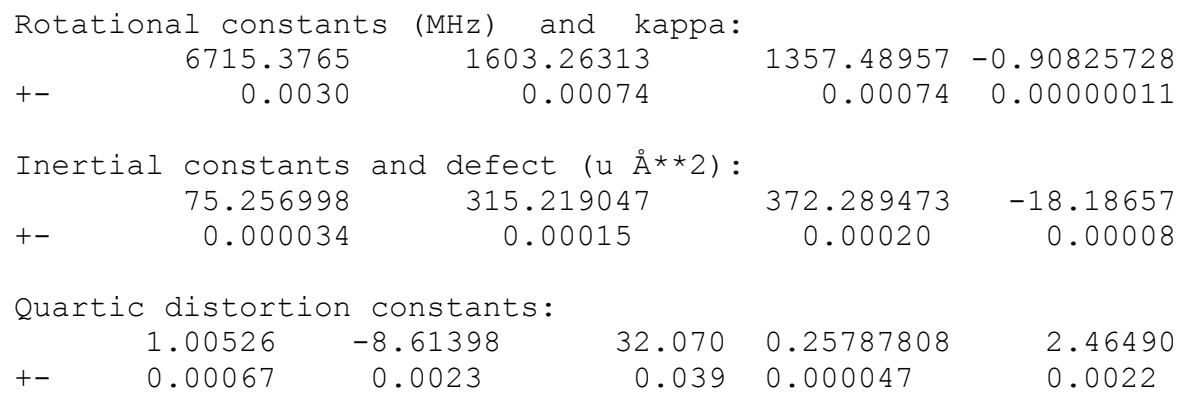




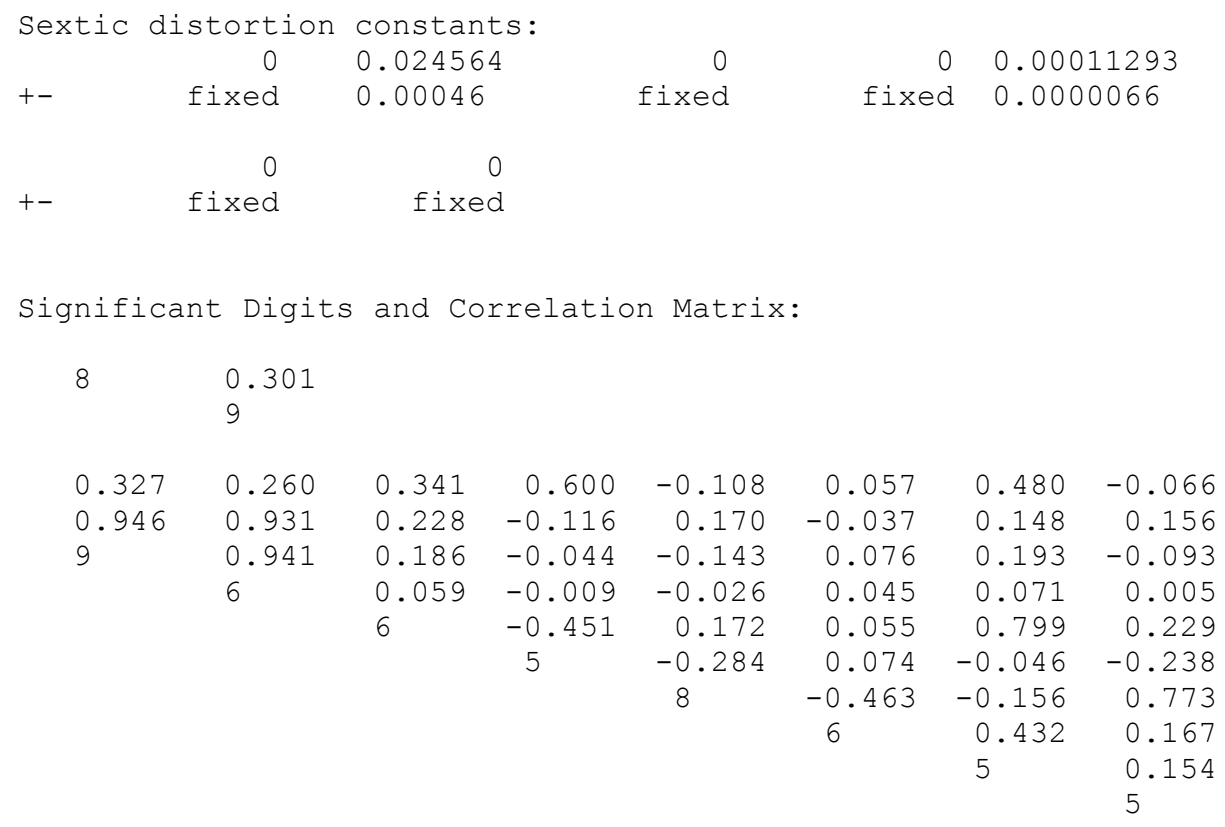


TABLE 4S: Microwave Spectrum of the First Excited State of the Torsion about the C3-C4 bond of Conformer IV of $\mathrm{H}^{80} \mathrm{SeCH}_{2} \mathrm{CH}_{2} \mathrm{C} \equiv \mathrm{CH}$

Total number of transitions used in the least-squares fit: 328

\begin{tabular}{|c|c|c|c|c|c|c|c|c|c|c|c|}
\hline & \multicolumn{4}{|c|}{ Transition } & \multicolumn{2}{|c|}{ obs. frequency } & \multirow{2}{*}{$\begin{array}{l}\text { obs.- } \\
\text { calc. } \\
0.003\end{array}$} & weight & t & \multicolumn{2}{|c|}{$\begin{array}{c}\text { distortion corre } \\
\text { total higher }\end{array}$} \\
\hline 10 & 5 & 6 & 10 & 6 & 5 & 58287.180 & & 0.20 & 0.0 & -12.645 & $3.2 E-3$ \\
\hline 10 & 5 & 5 & 10 & 6 & 4 & 58287.180 & 0.156 & 0.20 & 0.6 & -12.644 & $3.2 E-3$ \\
\hline 11 & 7 & 5 & 11 & 8 & 4 & 79503.460 & 0.068 & 0.15 & 0.4 & -41.586 & $6.4 E-3$ \\
\hline 11 & 7 & 4 & 11 & 8 & 3 & 79503.460 & 0.069 & 0.15 & 0.4 & -41.586 & $6.4 E-3$ \\
\hline 14 & 4 & 10 & 14 & 5 & 9 & 47291.750 & 0.035 & 0.15 & 0.2 & 5.066 & $9.7 E-3$ \\
\hline 14 & 6 & 9 & 14 & 7 & 8 & 68831.540 & -0.109 & 0.10 & -0.9 & -14.142 & 0.014 \\
\hline 14 & 6 & 8 & 14 & 7 & 7 & 68831.540 & -0.014 & 0.10 & -0.1 & -14.141 & 0.014 \\
\hline 14 & 0 & 14 & 15 & 1 & 15 & 42112.020 & -0.084 & 0.10 & -0.7 & -6.021 & 1.1E-4 \\
\hline 14 & 1 & 13 & 15 & 2 & 14 & 49090.140 & -0.189 & 0.10 & -1.6 & 1.146 & $3.4 E-3$ \\
\hline 14 & 6 & 9 & 15 & 6 & 10 & 44377.810 & 0.034 & 0.15 & 0.2 & -4.231 & 0.012 \\
\hline 14 & 6 & 8 & 15 & 6 & 9 & 44377.810 & -0.094 & 0.15 & -0.5 & -4.232 & 0.012 \\
\hline 14 & 7 & 8 & 15 & 7 & 9 & 44344.680 & -0.029 & 0.10 & -0.2 & -0.651 & 0.016 \\
\hline 14 & 7 & 7 & 15 & 7 & 8 & 44344.680 & -0.031 & 0.10 & -0.3 & -0.651 & 0.016 \\
\hline 15 & 7 & 9 & 15 & 8 & 8 & 79448.480 & 0.226 & 0.10 & 2.0 & -27.042 & 0.021 \\
\hline 15 & 7 & 8 & 15 & 8 & 7 & 79448.480 & 0.230 & 0.10 & 2.0 & -27.042 & 0.021 \\
\hline 15 & 0 & 15 & 16 & 1 & 16 & 44710.230 & 0.055 & 0.10 & 0.4 & -7.670 & $9.6 E-6$ \\
\hline 15 & 1 & 15 & 16 & 0 & 16 & 44153.250 & -0.160 & 0.10 & -1.3 & -10.097 & $-4 \cdot 3 E-4$ \\
\hline 15 & 1 & 14 & 16 & 2 & 15 & 50950.080 & -0.042 & 0.12 & -0.3 & 0.261 & $4.1 E-3$ \\
\hline 15 & 2 & 13 & 16 & 3 & 14 & 65311.460 & -0.061 & 0.15 & -0.3 & 8.739 & 0.010 \\
\hline 15 & 4 & 11 & 16 & 4 & 12 & 47650.500 & -0.085 & 0.10 & -0.7 & -13.775 & $6.2 E-3$ \\
\hline 15 & 5 & 11 & 16 & 5 & 12 & 47423.510 & 0.172 & 0.20 & 0.7 & -10.004 & $9.9 E-3$ \\
\hline 15 & 6 & 10 & 16 & 6 & 11 & 47354.340 & 0.212 & 0.10 & 1.7 & -6.615 & 0.014 \\
\hline 15 & 6 & 9 & 16 & 6 & 10 & 47354.340 & -0.056 & 0.10 & -0.5 & -6.619 & 0.014 \\
\hline 15 & 7 & 9 & 16 & 7 & 10 & 47313.310 & -0.145 & 0.15 & -0.8 & -2.755 & 0.020 \\
\hline 15 & 7 & 8 & 16 & 7 & 9 & 47313.310 & -0.151 & 0.15 & -0.8 & -2.756 & 0.020 \\
\hline 15 & 8 & 8 & 16 & 8 & 9 & 47289.400 & 0.063 & 0.10 & 0.5 & 1.607 & 0.025 \\
\hline 15 & 8 & 7 & 16 & 8 & 8 & 47289.400 & 0.063 & 0.10 & 0.5 & 1.607 & 0.025 \\
\hline 15 & 9 & 7 & 16 & 9 & 8 & 47275.010 & 0.105 & 0.10 & 0.8 & 6.500 & 0.032 \\
\hline 15 & 9 & 6 & 16 & 9 & 7 & 47275.010 & 0.105 & 0.10 & 0.8 & 6.500 & 0.032 \\
\hline 16 & 4 & 12 & 16 & 5 & 11 & 46906.370 & -0.035 & 0.15 & -0.2 & 11.896 & 0.016 \\
\hline 16 & 1 & 16 & 17 & 0 & 17 & 46932.920 & -0.007 & 0.10 & -0.1 & -11.652 & $-5.7 E-4$ \\
\hline 16 & 1 & 15 & 17 & 2 & 16 & 52885.610 & -0.201 & 0.10 & -1.6 & -1.194 & $4.8 E-3$ \\
\hline 16 & 5 & 11 & 17 & 5 & 12 & 50430.320 & 0.057 & 0.15 & 0.3 & -13.246 & 0.012 \\
\hline 16 & 7 & 10 & 17 & 7 & 11 & 50284.700 & 0.081 & 0.10 & 0.7 & -5.276 & 0.023 \\
\hline 16 & 7 & 9 & 17 & 7 & 10 & 50284.700 & 0.069 & 0.10 & 0.6 & -5.276 & 0.023 \\
\hline 16 & 8 & 9 & 17 & 8 & 10 & 50255.000 & -0.077 & 0.10 & -0.6 & -0.606 & 0.031 \\
\hline 16 & 8 & 8 & 17 & 8 & 9 & 50255.000 & -0.077 & 0.10 & -0.6 & -0.606 & 0.031 \\
\hline 17 & 4 & 13 & 17 & 5 & 12 & 46620.710 & -0.219 & 0.10 & -1.8 & 16.307 & 0.021 \\
\hline 17 & 7 & 11 & 17 & 8 & 10 & 79394.900 & 0.307 & 0.15 & 1.7 & -18.010 & 0.034 \\
\hline 17 & 7 & 10 & 17 & 8 & 9 & 79394.900 & 0.328 & 0.15 & 1.8 & -18.010 & 0.034 \\
\hline 17 & 0 & 17 & 18 & 1 & 18 & 49980.060 & 0.070 & 0.10 & 0.6 & -11.585 & $-3.6 E-4$ \\
\hline 17 & 1 & 16 & 18 & 2 & 17 & 54911.930 & -0.073 & 0.15 & -0.4 & -3.275 & $5.5 E-3$ \\
\hline 17 & 5 & 12 & 18 & 5 & 13 & 53436.890 & 0.131 & 0.10 & 1.1 & -16.935 & 0.014 \\
\hline 17 & 7 & 11 & 18 & 7 & 12 & 53258.320 & -0.039 & 0.10 & -0.3 & -8.242 & 0.028 \\
\hline 17 & 7 & 10 & 18 & 7 & 11 & 53258.320 & -0.066 & 0.10 & -0.5 & -8.243 & 0.028 \\
\hline 17 & 9 & 9 & 18 & 9 & 10 & 53200.590 & -0.067 & 0.10 & -0.5 & 2.303 & 0.046 \\
\hline 17 & 9 & 8 & 18 & 9 & 9 & 53200.590 & -0.067 & 0.10 & -0.5 & 2.303 & 0.046 \\
\hline 17 & 10 & 8 & 18 & 10 & 9 & 53187.090 & -0.153 & 0.10 & -1.2 & 8.458 & 0.057 \\
\hline 17 & 10 & 7 & 18 & 10 & 8 & 53187.090 & -0.153 & 0.10 & -1.2 & 8.458 & 0.057 \\
\hline 17 & 11 & 7 & 18 & 11 & 8 & 53179.470 & -0.136 & 0.10 & -1.1 & 15.226 & 0.069 \\
\hline 17 & 11 & 6 & 18 & 11 & 7 & 53179.470 & -0.136 & 0.10 & -1.1 & 15.226 & 0.069 \\
\hline
\end{tabular}




\begin{tabular}{|c|c|c|c|c|c|c|c|c|c|c|c|}
\hline 18 & 7 & 12 & 18 & 8 & 11 & 79358.850 & -0.049 & 0.15 & -0.3 & -13.024 & 0.043 \\
\hline 18 & 7 & 11 & 18 & 8 & 10 & 79358.850 & -0.001 & 0.15 & -0.0 & -13.023 & 0.043 \\
\hline 18 & 0 & 18 & 19 & 1 & 19 & 52640.370 & -0.187 & 0.10 & -1.5 & -13.858 & $-6.6 E-4$ \\
\hline 18 & 1 & 18 & 19 & 0 & 19 & 52434.870 & -0.249 & 0.10 & -2.0 & -15.407 & $-1 \cdot 0 E-3$ \\
\hline 18 & 2 & 17 & 19 & 1 & 18 & 52758.070 & 0.243 & 0.15 & 1.3 & -30.230 & $-2 \cdot 7 E-4$ \\
\hline 18 & 4 & 14 & 19 & 4 & 15 & 56920.870 & 0.161 & 0.10 & 1.3 & -27.714 & 0.010 \\
\hline 18 & 5 & 14 & 19 & 5 & 15 & 56413.820 & 0.058 & 0.10 & 0.5 & -20.714 & 0.017 \\
\hline 18 & 5 & 13 & 19 & 5 & 14 & 56452.630 & 0.151 & 0.10 & 1.2 & -21.212 & 0.016 \\
\hline 18 & 7 & 12 & 19 & 7 & 13 & 56234.900 & 0.064 & 0.10 & 0.5 & -11.687 & 0.033 \\
\hline 18 & 7 & 11 & 19 & 7 & 12 & 56234.900 & 0.007 & 0.10 & 0.1 & -11.688 & 0.033 \\
\hline 18 & 8 & 11 & 19 & 8 & 12 & 56192.410 & 0.197 & 0.10 & 1.6 & -6.370 & 0.043 \\
\hline 18 & 8 & 10 & 19 & 8 & 11 & 56192.410 & 0.196 & 0.10 & 1.6 & -6.370 & 0.043 \\
\hline 18 & 9 & 10 & 19 & 9 & 11 & 56165.650 & -0.006 & 0.10 & -0.0 & -0.466 & 0.054 \\
\hline 18 & 9 & 9 & 19 & 9 & 10 & 56165.650 & -0.006 & 0.10 & -0.0 & -0.466 & 0.054 \\
\hline 18 & 10 & 9 & 19 & 10 & 10 & 56149.320 & 0.173 & 0.10 & 1.4 & 6.057 & 0.067 \\
\hline 18 & 10 & 8 & 19 & 10 & 9 & 56149.320 & 0.173 & 0.10 & 1.4 & 6.057 & 0.067 \\
\hline 18 & 11 & 8 & 19 & 11 & 9 & 56139.390 & 0.028 & 0.10 & 0.2 & 13.221 & 0.081 \\
\hline 18 & 11 & 7 & 19 & 11 & 8 & 56139.390 & 0.028 & 0.10 & 0.2 & 13.221 & 0.081 \\
\hline 19 & 1 & 19 & 20 & 0 & 20 & 55166.790 & -0.069 & 0.10 & -0.6 & -17.640 & $-1 \cdot 4 E-3$ \\
\hline 19 & 2 & 18 & 20 & 1 & 19 & 55942.420 & -0.049 & 0.15 & -0.3 & -32.054 & $2.6 E-4$ \\
\hline 19 & 5 & 14 & 20 & 5 & 15 & 59479.120 & 0.016 & 0.10 & 0.1 & -26.151 & 0.019 \\
\hline 19 & 7 & 13 & 20 & 7 & 14 & 59214.290 & 0.077 & 0.10 & 0.6 & -15.644 & 0.038 \\
\hline 19 & 7 & 12 & 20 & 7 & 13 & 59214.290 & -0.036 & 0.10 & -0.3 & -15.646 & 0.038 \\
\hline 19 & 8 & 12 & 20 & 8 & 13 & 59164.050 & 0.215 & 0.10 & 1.7 & -9.980 & 0.050 \\
\hline 19 & 8 & 11 & 20 & 8 & 12 & 59164.050 & 0.212 & 0.10 & 1.7 & -9.980 & 0.050 \\
\hline 19 & 9 & 11 & 20 & 9 & 12 & 59131.790 & -0.388 & 0.20 & -1.5 & -3.721 & 0.063 \\
\hline 19 & 9 & 10 & 20 & 9 & 11 & 59131.790 & -0.388 & 0.20 & -1.5 & -3.721 & 0.063 \\
\hline 19 & 10 & 10 & 20 & 10 & 11 & 59112.370 & 0.180 & 0.15 & 1.0 & 3.178 & 0.078 \\
\hline 19 & 10 & 9 & 20 & 10 & 10 & 59112.370 & 0.180 & 0.15 & 1.0 & 3.178 & 0.078 \\
\hline 20 & 0 & 20 & 21 & 1 & 21 & 57992.690 & -0.211 & 0.10 & -1.7 & -19.067 & $-1 \cdot 5 E-3$ \\
\hline 20 & 1 & 20 & 21 & 0 & 21 & 57890.070 & 0.031 & 0.10 & 0.2 & -20.130 & $-1 \cdot 9 E-3$ \\
\hline 20 & 1 & 19 & 21 & 2 & 20 & 61564.000 & 0.053 & 0.10 & 0.4 & -13.059 & 7. $3 E-3$ \\
\hline 20 & 5 & 15 & 21 & 5 & 16 & 62518.370 & -0.318 & 0.20 & -1.3 & -31.840 & 0.022 \\
\hline 20 & 6 & 15 & 21 & 6 & 16 & 62291.620 & -0.128 & 0.15 & -0.7 & -25.655 & 0.032 \\
\hline 20 & 8 & 13 & 21 & 8 & 14 & 62137.720 & 0.074 & 0.10 & 0.6 & -14.119 & 0.058 \\
\hline 20 & 8 & 12 & 21 & 8 & 13 & 62137.720 & 0.068 & 0.10 & 0.5 & -14.119 & 0.058 \\
\hline 20 & 9 & 12 & 21 & 9 & 13 & 62100.360 & 0.056 & 0.10 & 0.4 & -7.492 & 0.073 \\
\hline 20 & 9 & 11 & 21 & 9 & 12 & 62100.360 & 0.056 & 0.10 & 0.4 & -7.492 & 0.073 \\
\hline 20 & 10 & 11 & 21 & 10 & 12 & 62076.380 & -0.050 & 0.10 & -0.4 & -0.208 & 0.090 \\
\hline 20 & 10 & 10 & 21 & 10 & 11 & 62076.380 & -0.050 & 0.10 & -0.4 & -0.208 & 0.090 \\
\hline 20 & 11 & 10 & 21 & 11 & 11 & 62061.400 & -0.090 & 0.10 & -0.7 & 7.764 & 0.109 \\
\hline 20 & 11 & 9 & 21 & 11 & 10 & 62061.400 & -0.090 & 0.10 & -0.7 & 7.764 & 0.109 \\
\hline 20 & 12 & 9 & 21 & 12 & 10 & 62052.820 & 0.004 & 0.10 & 0.0 & 16.442 & 0.130 \\
\hline 20 & 12 & 8 & 21 & 12 & 9 & 62052.820 & 0.004 & 0.10 & 0.0 & 16.442 & 0.130 \\
\hline 21 & 6 & 15 & 21 & 7 & 14 & 68390.120 & -0.046 & 0.15 & -0.2 & 17.561 & 0.068 \\
\hline 21 & 0 & 21 & 22 & 1 & 22 & 60679.340 & 0.081 & 0.10 & 0.6 & -22.025 & $-2 \cdot 2 E-3$ \\
\hline 21 & 1 & 21 & 22 & 0 & 22 & 60606.820 & -0.110 & 0.10 & -0.9 & -22.889 & $-2 \cdot 5 E-3$ \\
\hline 21 & 1 & 20 & 22 & 2 & 21 & 63952.320 & -0.029 & 0.10 & -0.2 & -17.271 & $7.8 E-3$ \\
\hline 21 & 2 & 20 & 22 & 1 & 21 & 62033.640 & 0.003 & 0.10 & 0.0 & -35.955 & $1.7 E-3$ \\
\hline 21 & 5 & 16 & 22 & 5 & 17 & 65573.800 & 0.129 & 0.10 & 1.0 & -38.382 & 0.025 \\
\hline 21 & 6 & 16 & 22 & 6 & 17 & 65291.540 & -0.051 & 0.10 & -0.4 & -31.131 & 0.037 \\
\hline 21 & 6 & 15 & 22 & 6 & 16 & 65300.920 & -0.015 & 0.10 & -0.1 & $-31 \cdot 322$ & 0.037 \\
\hline 21 & 8 & 14 & 22 & 8 & 15 & 65113.790 & 0.027 & 0.10 & 0.2 & -18.817 & 0.066 \\
\hline 21 & 8 & 13 & 22 & 8 & 14 & 65113.790 & 0.015 & 0.10 & 0.1 & -18.818 & 0.066 \\
\hline 21 & 9 & 13 & 22 & 9 & 14 & 65070.150 & 0.032 & 0.10 & 0.3 & -11.808 & 0.084 \\
\hline 21 & 9 & 12 & 22 & 9 & 13 & 65070.150 & 0.032 & 0.10 & 0.3 & -11.808 & 0.084 \\
\hline 21 & 10 & 12 & 22 & 10 & 13 & 65041.950 & 0.022 & 0.10 & 0.2 & -4.130 & 0.103 \\
\hline 21 & 10 & 11 & 22 & 10 & 12 & 65041.950 & 0.022 & 0.10 & 0.2 & -4.130 & 0.103 \\
\hline 21 & 11 & 11 & 22 & 11 & 12 & 65023.870 & -0.080 & 0.10 & -0.6 & 4.256 & 0.125 \\
\hline 21 & 11 & 10 & 22 & 11 & 11 & 65023.870 & -0.080 & 0.10 & -0.6 & 4.256 & 0.125 \\
\hline 21 & 12 & 10 & 22 & 12 & 11 & 65013.020 & -0.088 & 0.10 & -0.7 & 13.375 & 0.149 \\
\hline 21 & 12 & 9 & 22 & 12 & 10 & 65013.020 & -0.088 & 0.10 & -0.7 & 13.375 & 0.149 \\
\hline
\end{tabular}




\begin{tabular}{|c|c|c|c|c|c|c|c|c|c|c|c|}
\hline 21 & 14 & 8 & 22 & 14 & 9 & 65005.540 & -0.314 & 0.15 & -1.7 & 33.864 & 0.203 \\
\hline 21 & 14 & 7 & 22 & 14 & 8 & 65005.540 & -0.314 & 0.15 & -1.7 & 33.864 & 0.203 \\
\hline 22 & 6 & 16 & 22 & 7 & 15 & 68272.000 & 0.039 & 0.15 & 0.2 & 23.644 & 0.081 \\
\hline 22 & 0 & 22 & 23 & 1 & 23 & 63369.920 & 0.034 & 0.15 & 0.2 & -25.234 & $-3.0 E-3$ \\
\hline 22 & 1 & 22 & 23 & 0 & 23 & 63319.370 & 0.162 & 0.20 & 0.6 & -25.929 & $-3 \cdot 2 E-3$ \\
\hline 22 & 1 & 21 & 23 & 2 & 22 & 66408.160 & -0.022 & 0.15 & -0.1 & -21.829 & $8.3 E-3$ \\
\hline 22 & 5 & 17 & 23 & 5 & 18 & 68647.090 & 0.238 & 0.10 & 1.9 & -45.899 & 0.029 \\
\hline 22 & 8 & 15 & 23 & 8 & 16 & 68092.340 & 0.033 & 0.10 & 0.3 & -24.110 & 0.076 \\
\hline 22 & 8 & 14 & 23 & 8 & 15 & 68092.340 & 0.010 & 0.10 & 0.1 & -24.110 & 0.076 \\
\hline 22 & 9 & 14 & 23 & 9 & 15 & 68041.820 & 0.118 & 0.10 & 0.9 & -16.702 & 0.096 \\
\hline 22 & 9 & 13 & 23 & 9 & 14 & 68041.820 & 0.117 & 0.10 & 0.9 & -16.702 & 0.096 \\
\hline 22 & 10 & 13 & 23 & 10 & 14 & 68008.800 & 0.058 & 0.10 & 0.5 & -8.618 & 0.118 \\
\hline 22 & 10 & 12 & 23 & 10 & 13 & 68008.800 & 0.058 & 0.10 & 0.5 & -8.618 & 0.118 \\
\hline 22 & 11 & 12 & 23 & 11 & 13 & 67987.380 & -0.020 & 0.10 & -0.2 & 0.191 & 0.143 \\
\hline 22 & 11 & 11 & 23 & 11 & 12 & 67987.380 & -0.020 & 0.10 & -0.2 & 0.191 & 0.143 \\
\hline 22 & 12 & 11 & 23 & 12 & 12 & 67974.020 & -0.123 & 0.10 & -1.0 & 9.757 & 0.170 \\
\hline 22 & 12 & 10 & 23 & 12 & 11 & 67974.020 & -0.123 & 0.10 & -1.0 & 9.757 & 0.170 \\
\hline 22 & 13 & 10 & 23 & 13 & 11 & 67966.840 & 0.057 & 0.20 & 0.2 & 20.098 & 0.200 \\
\hline 22 & 13 & 9 & 23 & 13 & 10 & 67966.840 & 0.057 & 0.20 & 0.2 & 20.098 & 0.200 \\
\hline 23 & 2 & 22 & 23 & 3 & 21 & 42446.210 & -0.307 & 0.10 & -2.6 & -36.664 & 0.039 \\
\hline 23 & 4 & 19 & 23 & 5 & 18 & 42617.450 & 0.104 & 0.10 & 0.9 & 69.024 & 0.071 \\
\hline 23 & 5 & 19 & 23 & 6 & 18 & 57188.700 & 0.152 & 0.15 & 0.8 & 35.204 & 0.082 \\
\hline 23 & 0 & 23 & 24 & 1 & 24 & 66063.570 & 0.075 & 0.10 & 0.6 & -28.706 & $-3.9 E-3$ \\
\hline 23 & 2 & 22 & 24 & 1 & 23 & 67848.620 & 0.046 & 0.10 & 0.4 & -40.827 & $3.5 E-3$ \\
\hline 23 & 2 & 21 & 24 & 3 & 22 & 76315.880 & -0.285 & 0.10 & -2.4 & 9.180 & 0.035 \\
\hline 23 & 8 & 16 & 24 & 8 & 17 & 71073.530 & 0.130 & 0.10 & 1.0 & -30.031 & 0.086 \\
\hline 23 & 8 & 15 & 24 & 8 & 16 & 71073.530 & 0.085 & 0.10 & 0.7 & -30.032 & 0.086 \\
\hline 23 & 10 & 14 & 24 & 10 & 15 & 70976.950 & 0.015 & 0.10 & 0.1 & -13.702 & 0.134 \\
\hline 23 & 10 & 13 & 24 & 10 & 14 & 70976.950 & 0.015 & 0.10 & 0.1 & -13.702 & 0.134 \\
\hline 23 & 11 & 13 & 24 & 11 & 14 & 70952.000 & 0.116 & 0.10 & 0.9 & -4.459 & 0.163 \\
\hline 23 & 11 & 12 & 24 & 11 & 13 & 70952.000 & 0.116 & 0.10 & 0.9 & -4.459 & 0.163 \\
\hline 23 & 12 & 12 & 24 & 12 & 13 & 70935.990 & 0.035 & 0.15 & 0.2 & 5.561 & 0.194 \\
\hline 23 & 12 & 11 & 24 & 12 & 12 & 70935.990 & 0.035 & 0.15 & 0.2 & 5.561 & 0.194 \\
\hline 23 & 13 & 11 & 24 & 13 & 12 & 70926.470 & -0.185 & 0.10 & -1.5 & 16.381 & 0.227 \\
\hline 23 & 13 & 10 & 24 & 13 & 11 & 70926.470 & -0.185 & 0.10 & -1.5 & 16.381 & 0.227 \\
\hline 24 & 4 & 21 & 24 & 5 & 20 & 47311.790 & 0.011 & 0.10 & 0.1 & 24.599 & 0.075 \\
\hline 24 & 6 & 19 & 24 & 7 & 18 & 68028.530 & -0.337 & 0.10 & -2.7 & 36.305 & 0.114 \\
\hline 24 & 0 & 24 & 25 & 1 & 25 & 68759.150 & 0.004 & 0.10 & 0.0 & -32.455 & $-5.1 E-3$ \\
\hline 24 & 1 & 24 & 25 & 0 & 25 & 68734.520 & 0.021 & 0.10 & 0.2 & -32.894 & $-5 \cdot 3 E-3$ \\
\hline 24 & 2 & 22 & 25 & 3 & 23 & 77991.810 & 0.031 & 0.10 & 0.3 & 3.823 & 0.038 \\
\hline 24 & 8 & 17 & 25 & 8 & 18 & 74057.020 & -0.149 & 0.15 & -0.8 & -36.616 & 0.097 \\
\hline 24 & 8 & 16 & 25 & 8 & 17 & 74057.020 & -0.233 & 0.15 & -1.2 & -36.619 & 0.097 \\
\hline 24 & 10 & 15 & 25 & 10 & 16 & 73946.800 & 0.234 & 0.10 & 1.9 & -19.413 & 0.152 \\
\hline 24 & 10 & 14 & 25 & 10 & 15 & 73946.800 & 0.234 & 0.10 & 1.9 & -19.413 & 0.152 \\
\hline 24 & 11 & 14 & 25 & 11 & 15 & 73917.280 & -0.165 & 0.10 & $-1 \cdot 3$ & -9.726 & 0.184 \\
\hline 24 & 11 & 13 & 25 & 11 & 14 & 73917.280 & -0.165 & 0.10 & -1.3 & -9.726 & 0.184 \\
\hline 24 & 12 & 13 & 25 & 12 & 14 & 73898.700 & 0.125 & 0.15 & 0.7 & 0.757 & 0.219 \\
\hline 24 & 12 & 12 & 25 & 12 & 13 & 73898.700 & 0.125 & 0.15 & 0.7 & 0.757 & 0.219 \\
\hline 24 & 13 & 12 & 25 & 13 & 13 & 73886.970 & -0.158 & 0.20 & -0.6 & 12.063 & 0.257 \\
\hline 24 & 13 & 11 & 25 & 13 & 12 & 73886.970 & -0.158 & 0.20 & -0.6 & 12.063 & 0.257 \\
\hline 25 & 4 & 22 & 25 & 5 & 21 & 47563.900 & 0.003 & 0.10 & 0.0 & 22.996 & 0.088 \\
\hline 25 & 5 & 20 & 25 & 6 & 19 & 55718.970 & -0.148 & 0.10 & -1.2 & 65.473 & 0.117 \\
\hline 25 & 6 & 20 & 25 & 7 & 19 & 67872.440 & -0.304 & 0.20 & -1.2 & 43.477 & 0.134 \\
\hline 25 & 2 & 24 & 26 & 1 & 25 & 73485.530 & 0.059 & 0.10 & 0.5 & -47.177 & $5.4 E-3$ \\
\hline 25 & 2 & 23 & 26 & 3 & 24 & 79799.850 & -0.205 & 0.10 & -1.7 & -3.082 & 0.041 \\
\hline 25 & 9 & 17 & 26 & 9 & 18 & 76968.100 & 0.167 & 0.12 & 1.1 & -35.171 & 0.138 \\
\hline 25 & 9 & 16 & 26 & 9 & 17 & 76968.100 & 0.162 & 0.12 & 1.1 & -35.171 & 0.138 \\
\hline 25 & 10 & 16 & 26 & 10 & 17 & 76917.770 & 0.072 & 0.10 & 0.6 & -25.782 & 0.171 \\
\hline 25 & 10 & 15 & 26 & 10 & 16 & 76917.770 & 0.071 & 0.10 & 0.6 & -25.782 & 0.171 \\
\hline 25 & 11 & 15 & 26 & 11 & 16 & 76884.370 & 0.241 & 0.10 & 2.0 & -15.639 & 0.207 \\
\hline 25 & 11 & 14 & 26 & 11 & 15 & 76884.370 & 0.241 & 0.10 & 2.0 & -15.639 & 0.207 \\
\hline 25 & 12 & 14 & 26 & 12 & 15 & 76862.050 & 0.014 & 0.10 & 0.1 & -4.684 & 0.246 \\
\hline
\end{tabular}




\begin{tabular}{|c|c|c|c|c|c|c|c|c|c|c|}
\hline $25 \quad 12$ & 13 & 26 & 12 & 14 & 76862.050 & 0.014 & 0.10 & 0.1 & -4.684 & 0.246 \\
\hline 26 & 24 & 26 & 4 & 23 & 44937.360 & 0.068 & 0.10 & 0.6 & -32.230 & 0.075 \\
\hline 26 & 23 & 26 & 5 & 22 & 47911.700 & 0.056 & 0.20 & 0.2 & 19.829 & 0.101 \\
\hline 26 & 22 & 26 & 6 & 21 & 56753.590 & -0.188 & 0.10 & -1.5 & 49.999 & 0.131 \\
\hline 26 & 19 & 26 & 8 & 18 & 78740.390 & -0.053 & 0.10 & -0.4 & 40.669 & 0.180 \\
\hline 26 & 25 & 27 & 1 & 26 & 76260.430 & 0.157 & 0.10 & 1.3 & -50.993 & $6.4 E-3$ \\
\hline 10 & 17 & 27 & 10 & 18 & 79890.160 & -0.234 & 0.10 & -1.9 & -32.842 & 0.191 \\
\hline 10 & 16 & 27 & 10 & 17 & 79890.160 & -0.234 & 0.10 & -1.9 & -32.842 & 0.191 \\
\hline 13 & 14 & 27 & 13 & 15 & 79809.750 & -0.221 & 0.12 & -1.5 & 1.513 & 0.323 \\
\hline 13 & 13 & 27 & 13 & 14 & 79809.750 & -0.221 & 0.12 & -1.5 & 1.513 & 0.323 \\
\hline 27 & 26 & 27 & 3 & 25 & 50582.180 & -0.244 & 0.10 & -2.1 & -71.195 & 0.083 \\
\hline 27 & 24 & 27 & 5 & 23 & 48366.540 & 0.264 & 0.15 & 1.4 & 14.850 & 0.115 \\
\hline 27 & 21 & 27 & 7 & 20 & 67289.480 & -0.088 & 0.20 & -0.4 & 64.823 & 0.183 \\
\hline 27 & 27 & 28 & 0 & 28 & 76844.160 & -0.049 & 0.10 & -0.4 & -45.708 & -0.010 \\
\hline 27 & 26 & 28 & 1 & 27 & 79014.750 & -0.162 & 0.15 & -0.9 & -55.261 & 7. $3 E-3$ \\
\hline 2 & 27 & 28 & 3 & 26 & 52755.800 & -0.182 & 0.15 & -1.0 & -81.044 & 0.099 \\
\hline 28 & 26 & 28 & 4 & 25 & 47949.710 & 0.150 & 0.15 & 0.8 & -55.841 & 0.100 \\
\hline 28 & 23 & 28 & 6 & 22 & 53472.640 & 0.031 & 0.10 & 0.3 & 117.233 & 0.188 \\
\hline 28 & 23 & 28 & 7 & 22 & 67312.350 & -0.187 & 0.10 & -1.5 & 67.367 & 0.209 \\
\hline 6 & 22 & 28 & 7 & 21 & 66976.200 & -0.017 & 0.10 & -0.1 & 76.539 & 0.212 \\
\hline 2 & 27 & 29 & 3 & 26 & 40807.660 & -0.099 & 0.10 & -0.9 & -183.883 & 0.041 \\
\hline 29 & 23 & 29 & 7 & 22 & 66604.560 & 0.000 & 0.10 & 0.0 & 90.165 & 0.244 \\
\hline 30 & 29 & 30 & 3 & 28 & 57208.520 & -0.205 & 0.10 & -1.7 & -101.856 & 0.139 \\
\hline 30 & 27 & 30 & 5 & 26 & 50461.290 & 0.010 & 0.10 & 0.1 & -13.029 & 0.166 \\
\hline 30 & 26 & 30 & 6 & 25 & 56533.700 & 0.082 & 0.10 & 0.7 & 59.516 & 0.222 \\
\hline 5 & 25 & 30 & 6 & 24 & 51244.090 & 0.049 & 0.10 & 0.4 & 166.079 & 0.252 \\
\hline 6 & 25 & 30 & 7 & 24 & 66880.530 & -0.085 & 0.20 & -0.3 & 84.512 & 0.274 \\
\hline 30 & 24 & 30 & 7 & 23 & 66162.680 & 0.004 & 0.10 & 0.0 & 106.200 & 0.281 \\
\hline 30 & 23 & 30 & 8 & 22 & 78069.250 & -0.028 & 0.10 & -0.2 & 81.091 & 0.318 \\
\hline 31 & 29 & 31 & 3 & 28 & 46994.580 & -0.010 & 0.15 & -0.1 & -218.730 & 0.071 \\
\hline 31 & 29 & 31 & 4 & 28 & 53301.020 & -0.109 & 0.15 & -0.6 & -100.476 & 0.153 \\
\hline 31 & 28 & 31 & 5 & 27 & 51423.640 & 0.011 & 0.15 & 0.1 & -27.106 & 0.185 \\
\hline 31 & 27 & 31 & 6 & 26 & 56618.120 & 0.215 & 0.10 & 1.7 & 57.826 & 0.250 \\
\hline 31 & 25 & 31 & 7 & 24 & 65636.590 & -0.022 & 0.10 & -0.2 & 125.244 & 0.322 \\
\hline 31 & 25 & 31 & 8 & 24 & 77926.870 & -0.263 & 0.10 & -2.2 & 90.116 & 0.361 \\
\hline 32 & 31 & 32 & 3 & 30 & 61756.620 & -0.422 & 0.10 & -3.5 & -124.001 & 0.193 \\
\hline 32 & 30 & 32 & 4 & 29 & 55269.680 & 0.117 & 0.10 & 1.0 & -117.268 & 0.175 \\
\hline 32 & 29 & 32 & 5 & 28 & 52522.880 & 0.187 & 0.10 & 1.5 & -43.669 & 0.207 \\
\hline 32 & 28 & 32 & 6 & 27 & 56783.450 & 0.134 & 0.10 & 1.1 & 53.713 & 0.279 \\
\hline 32 & 27 & 32 & 7 & 26 & 66438.440 & -0.068 & 0.10 & -0.5 & 101.037 & 0.351 \\
\hline 32 & 26 & 32 & 8 & 25 & 77710.750 & -0.081 & 0.10 & -0.7 & 101.902 & 0.409 \\
\hline 32 & 25 & 32 & 8 & 24 & 77578.130 & -0.091 & 0.10 & -0.8 & 107.496 & 0.412 \\
\hline 33 & 31 & 33 & 3 & 30 & 53071.660 & -0.077 & 0.10 & -0.6 & -243.675 & 0.122 \\
\hline 33 & 29 & 33 & 6 & 28 & 57042.500 & 0.171 & 0.10 & 1.4 & 46.782 & 0.310 \\
\hline 33 & 28 & 33 & 7 & 27 & 66229.640 & -0.186 & 0.10 & -1.5 & 108.329 & 0.395 \\
\hline 33 & 27 & 33 & 7 & 26 & 64267.480 & -0.156 & 0.15 & -0.8 & 175.055 & 0.421 \\
\hline 33 & 26 & 33 & 8 & 25 & 77278.690 & 0.094 & 0.10 & 0.8 & 123.068 & 0.466 \\
\hline 34 & 31 & 34 & 4 & 30 & 40700.190 & -0.136 & 0.10 & -1.1 & -298.385 & 0.052 \\
\hline 34 & 29 & 34 & 6 & 28 & 45362.100 & -0.119 & 0.15 & -0.6 & 262.691 & 0.406 \\
\hline 34 & 28 & 34 & 7 & 27 & 63390.880 & 0.098 & 0.10 & 0.8 & 207.082 & 0.479 \\
\hline 34 & 27 & 34 & 8 & 26 & 76934.400 & 0.063 & 0.15 & 0.3 & 140.733 & 0.526 \\
\hline 35 & 34 & 35 & 3 & 33 & 68673.200 & -0.054 & 0.15 & -0.3 & -159.729 & 0.306 \\
\hline 35 & 33 & 35 & 4 & 32 & 61583.340 & 0.092 & 0.15 & 0.5 & -171.304 & 0.266 \\
\hline 35 & 32 & 35 & 4 & 31 & 43758.510 & -0.097 & 0.10 & -0.8 & -343.543 & 0.060 \\
\hline 35 & 32 & 35 & 5 & 31 & 56623.370 & -0.080 & 0.10 & -0.6 & -107.540 & 0.283 \\
\hline 35 & 31 & 35 & 6 & 30 & 57886.910 & 0.273 & 0.20 & 1.1 & 23.060 & 0.377 \\
\hline 35 & 30 & 35 & 6 & 29 & 43832.870 & -0.095 & 0.15 & -0.5 & 273.361 & 0.442 \\
\hline 35 & 28 & 35 & 8 & 27 & 76536.950 & -0.262 & 0.10 & -2.2 & 161.014 & 0.592 \\
\hline 36 & 34 & 36 & 4 & 33 & 63791.120 & 0.188 & 0.10 & 1.5 & -190.125 & 0.306 \\
\hline 36 & 32 & 36 & 6 & 31 & 58491.590 & 0.299 & 0.10 & 2.4 & 5.708 & 0.413 \\
\hline 36 & 31 & 36 & 6 & 30 & 42404.970 & -0.124 & 0.10 & -1.0 & 273.107 & 0.474 \\
\hline 6 & 31 & 36 & 7 & 30 & 65763.520 & 0.227 & 0.10 & 1.8 & 121.115 & 0.544 \\
\hline
\end{tabular}




\begin{tabular}{|c|c|c|c|c|c|c|c|c|c|c|}
\hline 36 & 730 & 36 & 8 & 29 & 76684.160 & 0.199 & 0.10 & 1.6 & 153.921 & 0.651 \\
\hline 36 & $7 \quad 29$ & 36 & 8 & 28 & 76077.130 & -0.128 & 0.10 & $-1 \cdot 1$ & 184.566 & 0.666 \\
\hline 37 & 235 & 37 & 3 & 34 & 64374.460 & 0.042 & 0.10 & 0.3 & -279.659 & 0.290 \\
\hline 37 & 334 & 37 & 4 & 33 & 50225.620 & 0.103 & 0.10 & 0.9 & -419.831 & 0.097 \\
\hline 37 & $4 \quad 34$ & 37 & 5 & 33 & 59975.170 & 0.243 & 0.20 & 1.0 & -160.151 & 0.349 \\
\hline 37 & 533 & 37 & 6 & 32 & 59228.180 & 0.560 & 0.20 & 2.2 & -15.551 & 0.450 \\
\hline 37 & 532 & 37 & 6 & 31 & 41148.730 & 0.027 & 0.10 & 0.2 & 259.455 & 0.498 \\
\hline 37 & 632 & 37 & 7 & 31 & 65698.030 & -0.213 & 0.15 & -1.1 & 120.555 & 0.600 \\
\hline 37 & 631 & 37 & 7 & 30 & 59831.740 & 0.014 & 0.15 & 0.1 & 333.076 & 0.697 \\
\hline 37 & 731 & 37 & 8 & 30 & 76397.620 & -0.017 & 0.10 & -0.1 & 167.352 & 0.724 \\
\hline 38 & 336 & 38 & 4 & 35 & 68309.370 & 0.217 & 0.10 & 1.8 & -228.495 & 0.402 \\
\hline 38 & $4 \quad 35$ & 38 & 5 & 34 & 61811.930 & 0.045 & 0.15 & 0.2 & -188.691 & 0.388 \\
\hline 38 & 533 & 38 & 6 & 32 & 40130.980 & -0.117 & 0.10 & -1.0 & 230.432 & 0.513 \\
\hline 38 & 633 & 38 & 7 & 32 & 65698.960 & -0.058 & 0.15 & -0.3 & 116.599 & 0.657 \\
\hline 38 & 632 & 38 & 7 & 31 & 58329.090 & -0.022 & 0.10 & -0.2 & 381.702 & 0.782 \\
\hline 38 & $7 \quad 32$ & 38 & 8 & 31 & 76107.230 & -0.042 & 0.10 & -0.3 & 180.388 & 0.803 \\
\hline 39 & 436 & 39 & 5 & 35 & 63743.100 & 0.022 & 0.10 & 0.2 & $-218 \cdot 327$ & 0.432 \\
\hline 39 & $4 \quad 35$ & 39 & 5 & 34 & 40602.210 & 0.100 & 0.10 & 0.8 & -369.340 & 0.108 \\
\hline 39 & 633 & 39 & 7 & 32 & 56692.890 & 0.108 & 0.10 & 0.9 & 429.648 & 0.871 \\
\hline 40 & 238 & 40 & 3 & 37 & 72167.620 & 0.181 & 0.10 & 1.5 & -310.818 & 0.483 \\
\hline 40 & 337 & 40 & 4 & 36 & 60015.600 & -0.042 & 0.10 & -0.3 & -490.008 & 0.230 \\
\hline 40 & 634 & 40 & 7 & 33 & 54958.290 & 0.016 & 0.10 & 0.1 & 473.089 & 0.960 \\
\hline 40 & $7 \quad 34$ & 40 & 8 & 33 & 75539.520 & -0.136 & 0.10 & -1.1 & 203.417 & 0.975 \\
\hline 40 & 733 & 40 & 8 & 32 & 73344.000 & -0.084 & 0.10 & -0.7 & 329.187 & 1.050 \\
\hline 41 & 239 & 41 & 3 & 38 & 74675.520 & 0.229 & 0.15 & 1.2 & -323.414 & 0.562 \\
\hline 41 & 338 & 41 & 4 & 37 & 63159.100 & -0.047 & 0.10 & -0.4 & -503.653 & 0.298 \\
\hline 41 & 636 & 41 & 7 & 35 & 66223.100 & 0.035 & 0.10 & 0.3 & 78.532 & 0.842 \\
\hline 41 & 735 & 41 & 8 & 34 & 75278.380 & 0.089 & 0.10 & 0.7 & 212.242 & 1.069 \\
\hline 41 & $7 \quad 34$ & 41 & 8 & 33 & 72358.130 & 0.162 & 0.10 & 1.3 & 382.811 & 1.175 \\
\hline 42 & 240 & 42 & 3 & 39 & 77150.350 & 0.178 & 0.10 & 1.5 & -337.229 & 0.649 \\
\hline 42 & 340 & 42 & 4 & 39 & 77580.450 & 0.173 & 0.10 & 1.5 & -307.686 & 0.680 \\
\hline 42 & 339 & 42 & 4 & 38 & 66214.560 & -0.161 & 0.10 & $-1 \cdot 3$ & -514.497 & 0.376 \\
\hline 42 & $4 \quad 38$ & 42 & 5 & 37 & 49276.890 & 0.054 & 0.15 & 0.3 & -628.002 & 0.078 \\
\hline 42 & 637 & 42 & 7 & 36 & 66611.170 & 0.014 & 0.15 & 0.1 & 55.436 & 0.906 \\
\hline 42 & 636 & 42 & 7 & 35 & 51404.980 & -0.024 & 0.15 & -0.1 & 528.181 & 1.123 \\
\hline 43 & $4 \quad 40$ & 43 & 5 & 39 & 72199.430 & 0.004 & 0.10 & 0.0 & -342.396 & 0.674 \\
\hline 43 & $4 \quad 39$ & 43 & 5 & 38 & 52596.950 & -0.092 & 0.10 & -0.8 & -701.764 & 0.091 \\
\hline 43 & 538 & 43 & 6 & 37 & 40403.220 & -0.030 & 0.10 & -0.2 & -173.374 & 0.411 \\
\hline 43 & 638 & 43 & 7 & 37 & 67123.000 & 0.041 & 0.15 & 0.2 & 26.523 & 0.971 \\
\hline 43 & $7 \quad 37$ & 43 & 8 & 36 & 74849.550 & 0.065 & 0.10 & 0.5 & 220.920 & 1.268 \\
\hline 43 & 736 & 43 & 8 & 35 & 69913.320 & 0.015 & 0.10 & 0.1 & 514.780 & 1.464 \\
\hline 44 & 341 & 44 & 4 & 40 & 72056.100 & -0.057 & 0.15 & -0.3 & -532.960 & 0.567 \\
\hline 44 & $4 \quad 41$ & 44 & 5 & 40 & 74445.970 & -0.080 & 0.10 & -0.7 & -373.751 & 0.755 \\
\hline 44 & 539 & 44 & 6 & 38 & 41734.730 & -0.024 & 0.10 & -0.2 & -303.071 & 0.354 \\
\hline 44 & $7 \quad 37$ & 44 & 8 & 36 & 68438.120 & 0.267 & 0.10 & 2.2 & 591.392 & 1.629 \\
\hline 45 & 342 & 45 & 4 & 41 & 74851.880 & -0.068 & 0.10 & -0.6 & -542.650 & 0.678 \\
\hline 45 & $4 \quad 42$ & 45 & 5 & 41 & 76727.800 & 0.006 & 0.10 & 0.0 & -404.987 & 0.847 \\
\hline 45 & $4 \quad 41$ & 45 & 5 & 40 & 59502.610 & -0.007 & 0.10 & -0.1 & -815.570 & 0.173 \\
\hline 45 & 540 & 45 & 6 & 39 & 43504.960 & -0.135 & 0.20 & -0.5 & -444.587 & 0.289 \\
\hline 45 & 639 & 45 & 7 & 38 & 46882.340 & -0.050 & 0.15 & -0.3 & 464.133 & 1.255 \\
\hline 45 & $7 \quad 39$ & 45 & 8 & 38 & 74622.920 & -0.075 & 0.10 & -0.6 & 212.127 & 1.480 \\
\hline 45 & 38 & 45 & 8 & 37 & 66796.570 & -0.039 & 0.15 & -0.2 & 671.795 & 1.803 \\
\hline 46 & 343 & 46 & 4 & 42 & 77575.420 & -0.126 & 0.10 & -1.1 & -553.594 & 0.800 \\
\hline 46 & 541 & 46 & 6 & 40 & 45693.910 & -0.142 & 0.10 & $-1 \cdot 2$ & -593.932 & 0.220 \\
\hline 46 & 640 & 46 & 7 & 39 & 45874.460 & -0.051 & 0.10 & -0.4 & 390.329 & 1.252 \\
\hline 46 & 740 & 46 & 8 & 39 & 74616.870 & 0.090 & 0.10 & 0.7 & 199.047 & 1.588 \\
\hline 47 & $4 \quad 43$ & 47 & 5 & 42 & 66401.550 & -0.132 & 0.10 & -1.1 & -882.103 & 0.345 \\
\hline 47 & $5 \quad 43$ & 47 & 6 & 42 & 73864.880 & 0.086 & 0.10 & 0.7 & -410.983 & 1.004 \\
\hline 47 & 641 & 47 & 7 & 40 & 45220.730 & 0.085 & 0.10 & 0.7 & 287.685 & 1.220 \\
\hline 47 & 740 & 47 & 8 & 39 & 63099.690 & -0.067 & 0.10 & -0.6 & 825.022 & 2.164 \\
\hline 48 & $4 \quad 44$ & 48 & 5 & 43 & 69752.470 & -0.054 & 0.10 & -0.4 & -900.888 & 0.466 \\
\hline 48 & $5 \quad 43$ & 48 & 6 & 42 & 51178.820 & -0.091 & 0.10 & -0.8 & -895.235 & 0.095 \\
\hline
\end{tabular}




$\begin{array}{rrrrrrrrrrrr}48 & 6 & 42 & 48 & 7 & 41 & 44968.560 & -0.099 & 0.15 & -0.5 & 156.204 & 1.157 \\ 49 & 5 & 45 & 49 & 6 & 44 & 78061.050 & 0.242 & 0.10 & 2.0 & -509.858 & 1.201 \\ 49 & 5 & 44 & 49 & 6 & 43 & 54370.050 & -0.044 & 0.15 & -0.2 & -1035.545 & 0.055 \\ 49 & 6 & 43 & 49 & 7 & 42 & 45154.420 & -0.205 & 0.10 & -1.7 & -3.166 & 1.064 \\ 50 & 4 & 46 & 50 & 5 & 45 & 76164.910 & 0.067 & 0.10 & 0.6 & -921.331 & 0.771 \\ 50 & 5 & 45 & 50 & 6 & 44 & 57775.960 & -0.136 & 0.10 & -1.1 & -1161.611 & 0.042 \\ 50 & 6 & 45 & 50 & 7 & 44 & 74567.700 & 0.142 & 0.10 & 1.2 & -335.429 & 1.478 \\ 50 & 6 & 44 & 50 & 7 & 43 & 45802.480 & 0.131 & 0.10 & 1.1 & -188.504 & 0.942 \\ 50 & 7 & 43 & 50 & 8 & 42 & 57198.570 & -0.138 & 0.15 & -0.8 & 935.447 & 2.611 \\ 51 & 6 & 46 & 51 & 7 & 45 & 76176.240 & 0.142 & 0.10 & 1.2 & -405.166 & 1.568 \\ 51 & 6 & 45 & 51 & 7 & 44 & 46923.170 & 0.147 & 0.10 & 1.2 & -396.754 & 0.794 \\ 51 & 7 & 44 & 51 & 8 & 43 & 55398.180 & 0.056 & 0.10 & 0.5 & 911.920 & 2.695 \\ 51 & 8 & 43 & 51 & 9 & 42 & 77108.160 & 0.125 & 0.15 & 0.7 & 961.256 & 3.369 \\ 52 & 5 & 47 & 52 & 6 & 46 & 64962.370 & -0.010 & 0.10 & -0.1 & -1355.878 & 0.124 \\ 52 & 6 & 47 & 52 & 7 & 46 & 77904.650 & 0.199 & 0.10 & 1.6 & -477.381 & 1.667 \\ 52 & 6 & 46 & 52 & 7 & 45 & 48514.700 & -0.033 & 0.15 & -0.2 & -623.477 & 0.626 \\ 52 & 8 & 44 & 52 & 9 & 43 & 75358.050 & -0.328 & 0.10 & -3.1 & 1085.805 & 3.696 \\ 53 & 5 & 48 & 53 & 6 & 47 & 68616.700 & 0.048 & 0.10 & 0.4 & -1421.015 & 0.230 \\ 53 & 6 & 47 & 53 & 7 & 46 & 50561.890 & 0.056 & 0.10 & 0.5 & -862.675 & 0.445 \\ 53 & 7 & 46 & 53 & 8 & 45 & 52495.320 & 0.065 & 0.15 & 0.4 & 742.138 & 2.711 \\ 54 & 5 & 49 & 54 & 6 & 48 & 72241.400 & -0.239 & 0.10 & -2.1 & -1465.999 & 0.382 \\ 54 & 6 & 48 & 54 & 7 & 47 & 53034.700 & 0.114 & 0.10 & 1.0 & -1106.835 & 0.263 \\ 56 & 5 & 51 & 56 & 6 & 50 & 79262.940 & -0.123 & 0.15 & -0.7 & -1508.144 & 0.815 \\ 56 & 7 & 49 & 56 & 8 & 48 & 50853.160 & -0.019 & 0.10 & -0.2 & 156.222 & 2.279 \\ 58 & 7 & 51 & 58 & 8 & 50 & 52068.100 & 0.120 & 0.10 & 1.0 & -435.992 & 1.689 \\ 59 & 6 & 53 & 59 & 7 & 52 & 69907.090 & -0.120 & 0.10 & -2.4 & -2104.677 & -0.139 \\ 60 & 7 & 53 & 60 & 8 & 52 & 55263.140 & 0.143 & 0.15 & 0.8 & -1140.856 & 0.924 \\ 61 & 8 & 53 & 61 & 9 & 52 & 58054.850 & -0.166 & 0.15 & -1.1 & 1097.467 & 5.240 \\ 62 & 8 & 54 & 62 & 9 & 53 & 57152.730 & -0.134 & 0.15 & -0.9 & 826.644 & 4.965\end{array}$

rms deviation: 1.2535

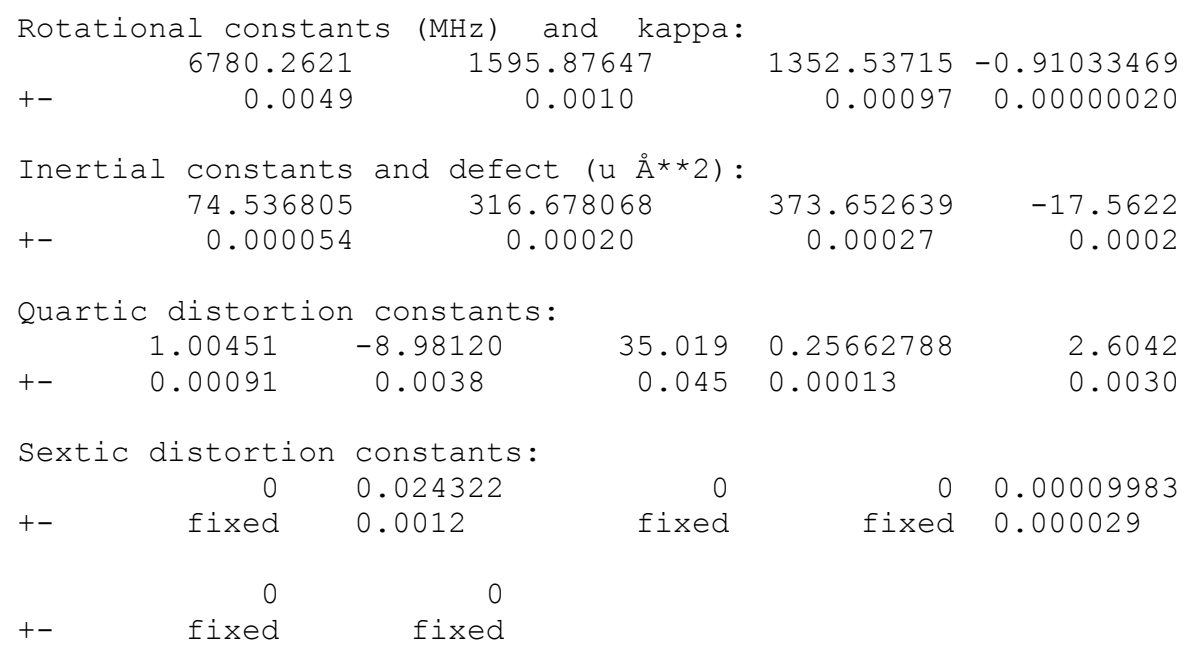

Significant Digits and Correlation Matrix:

$\begin{array}{llrrrrrr}8 & 0.280 & & & & & & \\ & 9 & & & & & & \\ & & & & & & & \\ 0.225 & 0.152 & 0.582 & 0.767 & 0.117 & 0.088 & 0.568 & 0.166 \\ 0.856 & 0.894 & 0.197 & -0.008 & 0.331 & 0.023 & 0.184 & 0.316 \\ 9 & 0.923 & 0.090 & 0.002 & -0.188 & 0.039 & 0.091 & -0.154 \\ & 6 & -0.048 & 0.008 & 0.012 & 0.037 & -0.020 & 0.023 \\ & & 6 & 0.053 & 0.244 & 0.168 & 0.922 & 0.311\end{array}$




$$
\begin{array}{ccll}
-0.031 & 0.038 & 0.185 & 0.014 \\
8 & -0.042 & 0.223 & 0.943 \\
& 5 & 0.323 & 0.231 \\
& & 5 & 0.355
\end{array}
$$


TABLE 5S: Microwave Spectrum of the Ground Vibrational State of Conformer IV of $\mathrm{H}^{78} \mathrm{SeCH}_{2} \mathrm{CH}_{2} \mathrm{C} \equiv \mathrm{CH}$

Total number of transitions used in the least-squares fit: 186

Transition obs. frequency

\begin{tabular}{|c|c|c|c|c|c|c|}
\hline 13 & 4 & 9 & 13 & 5 & 8 & 46727.340 \\
\hline 14 & 4 & 10 & 14 & 5 & 9 & 46591.160 \\
\hline 14 & 6 & 9 & 14 & 7 & 8 & 67863.280 \\
\hline 14 & 6 & 8 & 14 & 7 & 7 & 67863.280 \\
\hline 4 & 1 & 14 & 15 & 0 & 15 & 41823.340 \\
\hline 5 & 5 & 10 & 15 & 6 & 9 & 57254.270 \\
\hline 15 & 0 & 15 & 16 & 1 & 16 & 45148.730 \\
\hline 16 & 0 & 16 & 17 & 1 & 17 & 47808.500 \\
\hline 16 & 1 & 16 & 17 & 0 & 17 & 47452.300 \\
\hline 16 & 7 & 10 & 17 & 7 & 11 & 50883.330 \\
\hline 16 & 7 & 9 & 17 & 7 & 10 & 50883.330 \\
\hline 16 & 8 & 9 & 17 & 8 & 10 & 50851.320 \\
\hline 16 & 8 & 8 & 17 & 8 & 9 & 50851.320 \\
\hline 16 & 9 & 8 & 17 & 9 & 9 & 50831.890 \\
\hline 16 & 9 & 7 & 17 & 9 & 8 & 50831.890 \\
\hline 16 & 10 & 7 & 17 & 10 & 8 & 50820.050 \\
\hline 16 & 10 & 6 & 17 & 10 & 7 & 50820.050 \\
\hline 16 & 11 & 6 & 17 & 11 & 7 & 3.300 \\
\hline 16 & 11 & 5 & 17 & 11 & 6 & 50813.300 \\
\hline 17 & 5 & 12 & 17 & 6 & 11 & 57068.050 \\
\hline 17 & 0 & 17 & 18 & 1 & 18 & 50488.030 \\
\hline 17 & 7 & 11 & 18 & 7 & 12 & 53893.350 \\
\hline 17 & 7 & 10 & 18 & 7 & 11 & 53893.350 \\
\hline 17 & 8 & 10 & 18 & 8 & 11 & 53855.040 \\
\hline 17 & 8 & 9 & 18 & 8 & 10 & 53855.040 \\
\hline 17 & 9 & 9 & 18 & 9 & 10 & 53830.870 \\
\hline 17 & 9 & 8 & 18 & 9 & 9 & 53830.870 \\
\hline 17 & 10 & 8 & 18 & 10 & 9 & 53816.370 \\
\hline 17 & 10 & 7 & 18 & 10 & 8 & 53816.370 \\
\hline 17 & 11 & 7 & 18 & 11 & 8 & 53807.620 \\
\hline 17 & 11 & 6 & 18 & 11 & 7 & 53807.620 \\
\hline 18 & 5 & 13 & 18 & 6 & 12 & 56940.140 \\
\hline 18 & 0 & 18 & 19 & 1 & 19 & 53181.610 \\
\hline 18 & 6 & 12 & 19 & 6 & 13 & 56983.150 \\
\hline 18 & 7 & 12 & 19 & 7 & 13 & 56906.500 \\
\hline 18 & 7 & 11 & 19 & 7 & 12 & 56906.500 \\
\hline 18 & 8 & 11 & 19 & 8 & 12 & 56860.820 \\
\hline 18 & 8 & 10 & 19 & 8 & 11 & 56860.820 \\
\hline 18 & 9 & 10 & 19 & 9 & 11 & 56831.630 \\
\hline 18 & 9 & 9 & 19 & 9 & 10 & 56831.630 \\
\hline 18 & 11 & 8 & 19 & 11 & 9 & 56802.700 \\
\hline 18 & 11 & 7 & 19 & 11 & 8 & 56802.700 \\
\hline 18 & 12 & 7 & 19 & 12 & 8 & 56796.430 \\
\hline 18 & 12 & 6 & 19 & 12 & 7 & 56796.430 \\
\hline 19 & 4 & 15 & 19 & 5 & 14 & 44947.350 \\
\hline 19 & 6 & 14 & 20 & 6 & 15 & 60010.210 \\
\hline 19 & 6 & 13 & 20 & 6 & 14 & 60014.070 \\
\hline 19 & 7 & 13 & 20 & 7 & 14 & 59922.670 \\
\hline 19 & 7 & 12 & 20 & 7 & 13 & 59922.670 \\
\hline 19 & 9 & 11 & 20 & 9 & 12 & 59834.090 \\
\hline
\end{tabular}

obs.- weight $t$ calc.

$$
\begin{aligned}
& 0.256 \quad 0.15 \\
& -0.0750 .15 \\
& -0.2330 .15 \\
& -0.1150 .15 \\
& 0.0350 .10 \\
& 0.0450 .10 \\
& 0.1890 .10 \\
& -0.2550 .20 \\
& -0.0200 .10 \\
& 0.0390 .10 \\
& 0.0220 .10 \\
& -0.1480 .10 \\
& -0.1480 .10 \\
& 0.0010 .10 \\
& 0.0010 .10 \\
& 0.0380 .10 \\
& 0.0380 .10 \\
& -0.0170 .10 \\
& -0.0170 .10 \\
& 0.0010 .15 \\
& -0.1290 .10 \\
& -0.0080 .10 \\
& -0.0430 .10 \\
& 0.0760 .10 \\
& 0.0760 .10 \\
& -0.2000 .10 \\
& -0.2000 .10 \\
& 0.1130 .10 \\
& 0.1130 .10 \\
& 0.0940 .10 \\
& 0.0940 .10 \\
& -0.1130 .15 \\
& -0.2580 .10 \\
& -0.2040 .10 \\
& 0.1440 .15 \\
& 0.0720 .15 \\
& 0.2560 .15 \\
& 0.2550 .15 \\
& -0.1730 .10 \\
& -0.1730 .10 \\
& 0.0820 .10 \\
& 0.0820 .10 \\
& -0.1340 .10 \\
& -0.1340 .10 \\
& -0.1140 .10 \\
& 0.2880 .15 \\
& 0.1190 .15 \\
& 0.2080 .10 \\
& 0.0640 .10
\end{aligned}
$$

\begin{tabular}{|c|c|c|}
\hline 1.4 & 2.942 & $6.8 E-3$ \\
\hline-0.4 & 5.628 & $9.1 E-3$ \\
\hline$-1 \cdot 3$ & -11.979 & 0.013 \\
\hline-0.6 & -11.978 & 0.013 \\
\hline 0.3 & -8.728 & $2.8 E-4$ \\
\hline 0.4 & 1.751 & 0.014 \\
\hline 1.5 & -7.912 & $9.5 E-4$ \\
\hline$-1 \cdot 0$ & -9.786 & $1.1 E-3$ \\
\hline-0.2 & -11.705 & $6.5 E-4$ \\
\hline 0.3 & -6.128 & 0.022 \\
\hline 0.2 & -6.128 & 0.022 \\
\hline$-1 \cdot 2$ & -1.580 & 0.028 \\
\hline$-1 \cdot 2$ & -1.580 & 0.028 \\
\hline 0.0 & 3.499 & 0.036 \\
\hline 0.0 & 3.499 & 0.036 \\
\hline 0.3 & 9.131 & 0.044 \\
\hline 0.3 & 9.131 & 0.044 \\
\hline-0.1 & 15.328 & 0.053 \\
\hline-0.1 & 15.328 & 0.053 \\
\hline 0.0 & 8.905 & 0.023 \\
\hline-1.1 & -11.867 & 1. $4 E-3$ \\
\hline-0.1 & -9.205 & 0.026 \\
\hline-0.3 & -9.205 & 0.026 \\
\hline 0.6 & -4.344 & 0.033 \\
\hline 0.6 & -4.344 & 0.033 \\
\hline$-1 \cdot 6$ & 1.065 & 0.042 \\
\hline-1.6 & 1.065 & 0.042 \\
\hline 0.9 & 7.051 & 0.052 \\
\hline 0.9 & 7.051 & 0.052 \\
\hline 0.8 & 13.628 & 0.063 \\
\hline 0.8 & 13.628 & 0.063 \\
\hline-0.6 & 13.079 & 0.029 \\
\hline-2.1 & -14.160 & $1.6 E-3$ \\
\hline-1.6 & -17.495 & 0.022 \\
\hline 0.8 & -12.772 & 0.030 \\
\hline 0.4 & -12.773 & 0.030 \\
\hline 1.3 & -7.585 & 0.039 \\
\hline 1.3 & -7.585 & 0.039 \\
\hline$-1 \cdot 4$ & -1.836 & 0.050 \\
\hline-1.4 & -1.836 & 0.050 \\
\hline 0.7 & 11.473 & 0.075 \\
\hline 0.7 & 11.473 & 0.075 \\
\hline$-1 \cdot 1$ & 19.066 & 0.089 \\
\hline-1.1 & 19.066 & 0.089 \\
\hline-1.0 & 29.122 & 0.031 \\
\hline 1.5 & -21.898 & 0.025 \\
\hline 0.6 & -21.967 & 0.025 \\
\hline 1.7 & -16.864 & 0.035 \\
\hline 0.5 & -16.867 & 0.035 \\
\hline-0.7 & -5.235 & 0.058 \\
\hline
\end{tabular}$$
-0.0850 .10
$$

distortion corrections total higher 


\begin{tabular}{|c|c|c|c|c|c|c|c|c|c|c|c|}
\hline 19 & 9 & 10 & 20 & 9 & 11 & 59834.090 & -0.085 & 0.10 & -0.7 & -5.235 & 0.058 \\
\hline 19 & 10 & 10 & 20 & 10 & 11 & 59812.340 & 0.032 & 0.10 & 0.3 & 1.479 & 0.072 \\
\hline 19 & 10 & 9 & 20 & 10 & 10 & 59812.340 & 0.032 & 0.10 & 0.3 & 1.479 & 0.072 \\
\hline 19 & 12 & 8 & 20 & 12 & 9 & 59790.890 & 0.160 & 0.15 & 0.8 & 16.847 & 0.104 \\
\hline 19 & 12 & 7 & 20 & 12 & 8 & 59790.890 & 0.160 & 0.15 & 0.8 & 16.847 & 0.104 \\
\hline 20 & 0 & 20 & 21 & 1 & 21 & 58598.070 & 0.237 & 0.10 & 1.9 & -19.419 & $2.3 E-3$ \\
\hline 20 & 1 & 20 & 21 & 0 & 21 & 58509.670 & 0.058 & 0.12 & 0.4 & -20.346 & $2.0 E-3$ \\
\hline 20 & 8 & 13 & 21 & 8 & 14 & 62878.500 & -0.068 & 0.15 & -0.4 & -15.622 & 0.053 \\
\hline 20 & 8 & 12 & 21 & 8 & 13 & 62878.500 & -0.075 & 0.15 & -0.4 & -15.622 & 0.053 \\
\hline 20 & 9 & 12 & 21 & 9 & 13 & 62838.220 & -0.055 & 0.12 & -0.4 & -9.161 & 0.067 \\
\hline 20 & 9 & 11 & 21 & 9 & 12 & 62838.220 & -0.055 & 0.12 & -0.4 & -9.161 & 0.067 \\
\hline 21 & 4 & 17 & 21 & 5 & 16 & 43532.620 & -0.001 & 0.10 & -0.0 & 46.955 & 0.048 \\
\hline 21 & 4 & 17 & 21 & 5 & 16 & 43532.620 & -0.001 & 0.12 & -0.0 & 46.955 & 0.048 \\
\hline 21 & 11 & 11 & 22 & 11 & 12 & 65793.450 & -0.222 & 0.20 & -0.9 & 1.993 & 0.116 \\
\hline 21 & 11 & 10 & 22 & 11 & 11 & 65793.450 & -0.222 & 0.20 & -0.9 & 1.993 & 0.116 \\
\hline 22 & 2 & 21 & 22 & 3 & 20 & 40798.140 & 0.001 & 0.15 & 0.0 & -31.095 & 0.022 \\
\hline 22 & 3 & 20 & 22 & 4 & 19 & 40262.890 & 0.022 & 0.15 & 0.1 & -4.245 & 0.033 \\
\hline 22 & 0 & 22 & 23 & 1 & 23 & 64036.180 & 0.234 & 0.10 & 1.9 & -25.649 & $3.2 E-3$ \\
\hline 22 & 1 & 22 & 23 & 0 & 23 & 63993.110 & -0.085 & 0.10 & -0.7 & -26.246 & $3.0 E-3$ \\
\hline 22 & 7 & 15 & 23 & 7 & 16 & 68992.270 & 0.154 & 0.10 & 1.2 & -32.670 & 0.053 \\
\hline 22 & 8 & 15 & 23 & 8 & 16 & 68906.470 & -0.018 & 0.10 & -0.1 & -25.958 & 0.069 \\
\hline 22 & 8 & 14 & 23 & 8 & 15 & 68906.470 & -0.049 & 0.10 & -0.4 & -25.959 & 0.069 \\
\hline 22 & 9 & 14 & 23 & 9 & 15 & 68852.060 & 0.045 & 0.10 & 0.4 & $-18 \cdot 722$ & 0.088 \\
\hline 22 & 9 & 13 & 23 & 9 & 14 & 68852.060 & 0.044 & 0.10 & 0.4 & $-18 \cdot 722$ & 0.088 \\
\hline 22 & 10 & 13 & 23 & 10 & 14 & 68816.340 & 0.087 & 0.10 & 0.7 & -10.843 & 0.109 \\
\hline 22 & 10 & 12 & 23 & 10 & 13 & 68816.340 & 0.087 & 0.10 & 0.7 & -10.843 & 0.109 \\
\hline 22 & 11 & 12 & 23 & 11 & 13 & 68792.690 & -0.086 & 0.10 & -0.7 & -2.268 & 0.132 \\
\hline 22 & 11 & 11 & 23 & 11 & 12 & 68792.690 & -0.086 & 0.10 & -0.7 & -2.268 & 0.132 \\
\hline 22 & 12 & 11 & 23 & 12 & 12 & 68777.770 & -0.052 & 0.10 & -0.4 & 7.037 & 0.157 \\
\hline 22 & 12 & 10 & 23 & 12 & 11 & 68777.770 & -0.052 & 0.10 & -0.4 & 7.037 & 0.157 \\
\hline 23 & 5 & 19 & 23 & 6 & 18 & 56321.300 & -0.154 & 0.15 & -0.8 & 35.358 & 0.076 \\
\hline 23 & 1 & 23 & 24 & 0 & 24 & 66729.770 & -0.121 & 0.10 & $-1 \cdot 0$ & -29.632 & $3.6 E-3$ \\
\hline 23 & 1 & 22 & 24 & 2 & 23 & 69553.200 & 0.062 & 0.10 & 0.5 & -28.033 & 0.013 \\
\hline 23 & 8 & 16 & 24 & 8 & 17 & 71924.700 & 0.206 & 0.10 & 1.6 & -32.075 & 0.079 \\
\hline 23 & 8 & 15 & 24 & 8 & 16 & 71924.700 & 0.147 & 0.10 & 1.2 & -32.077 & 0.079 \\
\hline 23 & 9 & 15 & 24 & 9 & 16 & 71861.620 & -0.218 & 0.10 & -1.7 & -24.423 & 0.100 \\
\hline 23 & 9 & 14 & 24 & 9 & 15 & 71861.620 & -0.220 & 0.10 & -1.8 & -24.423 & 0.100 \\
\hline 23 & 11 & 13 & 24 & 11 & 14 & 71793.090 & 0.088 & 0.10 & 0.7 & -7.128 & 0.150 \\
\hline 23 & 11 & 12 & 24 & 11 & 13 & 71793.090 & 0.088 & 0.10 & 0.7 & -7.128 & 0.150 \\
\hline 24 & 2 & 23 & 24 & 3 & 22 & 44666.590 & -0.014 & 0.10 & -0.1 & -46.162 & 0.031 \\
\hline 24 & 3 & 22 & 24 & 4 & 21 & 42362.980 & 0.064 & 0.10 & 0.5 & -17.267 & 0.043 \\
\hline 24 & 3 & 22 & 24 & 4 & 21 & 42362.980 & 0.064 & 0.10 & 0.5 & -17.267 & 0.043 \\
\hline 24 & 4 & 20 & 24 & 5 & 19 & 40350.300 & -0.108 & 0.15 & -0.6 & 83.555 & 0.086 \\
\hline 24 & 5 & 19 & 24 & 6 & 18 & 55221.090 & 0.044 & 0.10 & 0.4 & 55.413 & 0.095 \\
\hline 24 & 6 & 18 & 24 & 7 & 17 & 66937.040 & 0.264 & 0.10 & 2.2 & 38.945 & 0.107 \\
\hline 24 & 1 & 24 & 25 & 0 & 25 & 69464.220 & -0.143 & 0.10 & -1.2 & -33.322 & $4.3 E-3$ \\
\hline 24 & 1 & 23 & 25 & 2 & 24 & 72154.620 & 0.009 & 0.10 & 0.1 & -33.114 & 0.015 \\
\hline 24 & 8 & 17 & 25 & 8 & 18 & 74945.700 & 0.321 & 0.20 & 1.3 & -38.874 & 0.089 \\
\hline 24 & 8 & 16 & 25 & 8 & 17 & 74945.700 & 0.210 & 0.20 & 0.8 & -38.878 & 0.089 \\
\hline 24 & 9 & 16 & 25 & 9 & 17 & 74873.880 & 0.125 & 0.10 & 1.0 & -30.781 & 0.113 \\
\hline 24 & 9 & 15 & 25 & 9 & 16 & 74873.880 & 0.122 & 0.10 & 1.0 & -30.782 & 0.113 \\
\hline 24 & 10 & 15 & 25 & 10 & 16 & 74826.160 & -0.062 & 0.10 & -0.5 & -22.058 & 0.140 \\
\hline 24 & 10 & 14 & 25 & 10 & 15 & 74826.160 & -0.063 & 0.10 & -0.5 & -22.058 & 0.140 \\
\hline 25 & 0 & 25 & 26 & 1 & 26 & 72211.350 & 0.082 & 0.10 & 0.7 & -37.039 & $5.2 E-3$ \\
\hline 25 & 1 & 25 & 26 & 0 & 26 & 72197.050 & -0.130 & 0.10 & -1.1 & -37.327 & $5.0 E-3$ \\
\hline 25 & 8 & 18 & 26 & 8 & 19 & 77969.520 & 0.238 & 0.10 & 1.9 & -46.392 & 0.099 \\
\hline 25 & 8 & 17 & 26 & 8 & 18 & 77969.520 & 0.037 & 0.10 & 0.3 & -46.400 & 0.099 \\
\hline 25 & 9 & 17 & 26 & 9 & 18 & 77887.940 & 0.078 & 0.10 & 0.6 & -37.833 & 0.127 \\
\hline 25 & 9 & 16 & 26 & 9 & 17 & 77887.940 & 0.072 & 0.10 & 0.6 & -37.834 & 0.127 \\
\hline 25 & 10 & 16 & 26 & 10 & 17 & 77833.490 & -0.119 & 0.10 & -1.0 & -28.661 & 0.157 \\
\hline 25 & 10 & 15 & 26 & 10 & 16 & 77833.490 & -0.119 & 0.10 & -1.0 & -28.661 & 0.157 \\
\hline 25 & 11 & 15 & 26 & 11 & 16 & 77797.020 & 0.004 & 0.10 & 0.0 & -18.772 & 0.191 \\
\hline
\end{tabular}




\begin{tabular}{|c|c|c|c|c|c|c|c|c|c|c|c|}
\hline 25 & 11 & 14 & 26 & 11 & 15 & 77797.020 & 0.004 & 0.10 & 0.0 & -18.772 & 0.191 \\
\hline 25 & 12 & 14 & 26 & 12 & 15 & 77772.350 & -0.201 & 0.10 & -1.7 & -8.105 & 0.227 \\
\hline 25 & 12 & 13 & 26 & 12 & 14 & 77772.350 & -0.201 & 0.10 & -1.7 & -8.105 & 0.227 \\
\hline 26 & 3 & 24 & 26 & 4 & 23 & 45004.910 & 0.038 & 0.10 & 0.3 & -35.992 & 0.055 \\
\hline 26 & 4 & 23 & 26 & 5 & 22 & 47448.620 & 0.075 & 0.15 & 0.4 & 16.348 & 0.088 \\
\hline 27 & 2 & 26 & 27 & 3 & 25 & 50989.620 & 0.074 & 0.15 & 0.4 & -72.968 & 0.051 \\
\hline 27 & 5 & 22 & 27 & 6 & 21 & 53162.870 & -0.060 & 0.10 & -0.5 & 100.968 & 0.161 \\
\hline 28 & 1 & 27 & 28 & 2 & 26 & 51643.910 & -0.229 & 0.10 & -1.9 & -112.966 & 0.039 \\
\hline 28 & 6 & 23 & 28 & 7 & 22 & 66258.020 & -0.088 & 0.10 & -0.7 & 67.819 & 0.197 \\
\hline 28 & 7 & 21 & 28 & 8 & 20 & 77258.960 & -0.079 & 0.15 & -0.4 & 61.252 & 0.227 \\
\hline 29 & 2 & 28 & 29 & 3 & 27 & 55435.440 & -0.008 & 0.15 & -0.0 & -92.980 & 0.071 \\
\hline 29 & 5 & 24 & 29 & 6 & 23 & 51064.910 & 0.004 & 0.10 & 0.0 & 145.640 & 0.224 \\
\hline 30 & 2 & 29 & 30 & 3 & 28 & 57702.590 & -0.071 & 0.10 & -0.6 & -103.515 & 0.084 \\
\hline 30 & 2 & 28 & 30 & 3 & 27 & 45046.020 & 0.155 & 0.10 & 1.3 & -201.366 & $-9.0 E-3$ \\
\hline 31 & 3 & 29 & 31 & 4 & 28 & 53702.820 & -0.083 & 0.10 & -0.7 & -105.300 & 0.095 \\
\hline 31 & 4 & 28 & 31 & 5 & 27 & 51329.120 & -0.017 & 0.10 & -0.1 & -35.242 & 0.143 \\
\hline 31 & 6 & 26 & 31 & 7 & 25 & 65592.770 & -0.008 & 0.10 & -0.1 & 92.134 & 0.293 \\
\hline 31 & 7 & 25 & 31 & 8 & 24 & 76710.280 & -0.162 & 0.10 & -1.5 & 91.548 & 0.340 \\
\hline 31 & 7 & 24 & 31 & 8 & 23 & 76600.030 & -0.142 & 0.15 & -0.8 & 95.912 & 0.343 \\
\hline 32 & 4 & 29 & 32 & 5 & 28 & 52516.770 & 0.237 & 0.10 & 1.9 & -52.724 & 0.155 \\
\hline 32 & 5 & 27 & 32 & 6 & 26 & 46855.260 & -0.202 & 0.15 & -1.1 & 221.752 & 0.346 \\
\hline 32 & 6 & 26 & 32 & 7 & 25 & 63664.410 & 0.320 & 0.15 & 1.7 & 154.575 & 0.365 \\
\hline 33 & 2 & 31 & 33 & 3 & 30 & 54177.540 & 0.002 & 0.10 & 0.0 & -237.555 & 0.026 \\
\hline 33 & 3 & 31 & 33 & 4 & 30 & 57823.770 & -0.073 & 0.10 & -0.6 & -139.409 & 0.120 \\
\hline 33 & 5 & 29 & 33 & 6 & 28 & 56453.610 & 0.026 & 0.15 & 0.1 & 37.992 & 0.272 \\
\hline 33 & 5 & 28 & 33 & 6 & 27 & 45289.040 & -0.087 & 0.10 & -0.7 & 242.092 & 0.388 \\
\hline 33 & 7 & 27 & 33 & 8 & 26 & 76235.910 & 0.082 & 0.15 & 0.5 & 115.327 & 0.437 \\
\hline 34 & 2 & 32 & 34 & 3 & 31 & 57082.730 & 0.114 & 0.15 & 0.6 & -246.516 & 0.046 \\
\hline 34 & 3 & 31 & 34 & 4 & 30 & 42076.760 & -0.013 & 0.15 & -0.1 & -310.968 & -0.069 \\
\hline 34 & 4 & 31 & 34 & 5 & 30 & 55301.040 & -0.020 & 0.15 & -0.1 & -94.610 & 0.177 \\
\hline 34 & 5 & 29 & 34 & 6 & 28 & 43732.160 & -0.066 & 0.10 & -0.6 & 255.483 & 0.428 \\
\hline 34 & 6 & 29 & 34 & 7 & 28 & 65005.540 & 0.070 & 0.10 & 0.6 & 110.589 & 0.413 \\
\hline 35 & 4 & 32 & 35 & 5 & 31 & 56887.520 & -0.117 & 0.15 & -0.6 & -118.666 & 0.189 \\
\hline 35 & 5 & 31 & 35 & 6 & 30 & 57456.980 & -0.017 & 0.15 & -0.1 & 10.490 & 0.317 \\
\hline 35 & 6 & 30 & 35 & 7 & 29 & 64870.750 & -0.009 & 0.20 & -0.0 & 113.458 & 0.458 \\
\hline 35 & 6 & 29 & 35 & 7 & 28 & 60722.250 & -0.050 & 0.10 & -0.4 & 256.663 & 0.563 \\
\hline 35 & 7 & 29 & 35 & 8 & 28 & 75697.970 & -0.122 & 0.20 & -0.5 & 140.783 & 0.552 \\
\hline 36 & 2 & 35 & 36 & 3 & 34 & 71591.870 & -0.035 & 0.10 & -0.5 & -174.045 & 0.218 \\
\hline 36 & 5 & 31 & 36 & 6 & 30 & 40928.410 & -0.086 & 0.10 & -0.7 & 250.274 & 0.484 \\
\hline 36 & 6 & 31 & 36 & 7 & 30 & 64786.340 & -0.004 & 0.15 & -0.0 & 113.773 & 0.504 \\
\hline 36 & 7 & 30 & 36 & 8 & 29 & 75411.950 & -0.085 & 0.10 & -0.7 & 153.689 & 0.617 \\
\hline 37 & 3 & 34 & 37 & 4 & 33 & 51829.170 & -0.034 & 0.10 & -0.3 & -418.250 & -0.088 \\
\hline 37 & 4 & 34 & 37 & 5 & 33 & 60409.800 & 0.109 & 0.10 & 0.9 & -171.768 & 0.216 \\
\hline 37 & 6 & 31 & 37 & 7 & 30 & 57953.650 & 0.141 & 0.10 & 1.2 & 346.622 & 0.740 \\
\hline 37 & 7 & 31 & 37 & 8 & 30 & 75120.270 & 0.097 & 0.15 & 0.5 & 166.320 & 0.687 \\
\hline 38 & 5 & 34 & 38 & 6 & 33 & 59967.600 & 0.324 & 0.15 & 1.7 & -59.306 & 0.377 \\
\hline 38 & 6 & 33 & 38 & 7 & 32 & 64820.970 & -0.087 & 0.15 & -0.5 & 104.335 & 0.596 \\
\hline 38 & 6 & 32 & 38 & 7 & 31 & 56343.120 & -0.008 & 0.10 & -0.1 & 393.298 & 0.838 \\
\hline 38 & 7 & 32 & 38 & 8 & 31 & 74828.380 & 0.025 & 0.15 & 0.1 & 178.266 & 0.761 \\
\hline 39 & 5 & 35 & 39 & 6 & 34 & 61088.770 & 0.175 & 0.15 & 0.9 & -90.279 & 0.394 \\
\hline 39 & 6 & 34 & 39 & 7 & 33 & 64966.870 & 0.003 & 0.15 & 0.0 & 93.392 & 0.642 \\
\hline 39 & 7 & 32 & 39 & 8 & 31 & 72535.370 & 0.065 & 0.10 & 0.6 & 298.653 & 0.944 \\
\hline 40 & 4 & 36 & 40 & 5 & 35 & 44788.500 & -0.236 & 0.10 & -2.0 & -497.922 & -0.184 \\
\hline 40 & 6 & 34 & 40 & 7 & 33 & 52834.680 & 0.157 & 0.10 & 1.3 & 472.337 & 1.035 \\
\hline 41 & 5 & 37 & 41 & 6 & 36 & 63754.620 & 0.096 & 0.10 & 0.8 & -162.682 & 0.425 \\
\hline 41 & 7 & 34 & 41 & 8 & 33 & 70467.950 & -0.304 & 0.20 & -1.2 & 406.146 & 1.217 \\
\hline 42 & 4 & 38 & 42 & 5 & 37 & 51212.340 & -0.084 & 0.10 & -0.7 & -651.829 & -0.290 \\
\hline 43 & 5 & 39 & 43 & 6 & 38 & 66951.410 & 0.091 & 0.10 & 0.7 & -246.569 & 0.455 \\
\hline 43 & 5 & 38 & 43 & 6 & 37 & 40881.910 & 0.045 & 0.10 & 0.4 & -264.894 & 0.135 \\
\hline 43 & 6 & 37 & 43 & 7 & 36 & 47751.590 & 0.057 & 0.12 & 0.4 & 486.364 & 1.234 \\
\hline 44 & 7 & 38 & 44 & 8 & 37 & 73576.250 & 0.299 & 0.15 & 1.6 & 200.616 & 1.267 \\
\hline 44 & 7 & 37 & 44 & 8 & 36 & 66114.380 & 0.031 & 0.10 & 0.3 & 621.798 & 1.759 \\
\hline
\end{tabular}




$\begin{array}{rrrrrrrrrrrr}45 & 5 & 40 & 45 & 6 & 39 & 44730.780 & -0.235 & 0.10 & -2.0 & -542.944 & -0.159 \\ 45 & 6 & 39 & 45 & 7 & 38 & 45337.400 & -0.207 & 0.10 & -1.8 & 379.134 & 1.215 \\ 46 & 5 & 42 & 46 & 6 & 41 & 72594.980 & -0.054 & 0.20 & -0.2 & -385.608 & 0.510 \\ 46 & 6 & 41 & 46 & 7 & 40 & 69407.650 & 0.290 & 0.10 & 2.5 & -136.358 & 0.872 \\ 46 & 7 & 40 & 46 & 8 & 39 & 73627.320 & 0.273 & 0.15 & 1.5 & 170.347 & 1.430 \\ 47 & 4 & 43 & 47 & 5 & 42 & 68459.470 & 0.069 & 0.10 & 0.8 & -852.673 & -0.241 \\ 47 & 5 & 42 & 47 & 6 & 41 & 50165.310 & -0.115 & 0.20 & -0.5 & -834.163 & -0.490 \\ 47 & 7 & 40 & 47 & 8 & 39 & 60444.660 & -0.230 & 0.10 & -2.7 & 830.423 & 2.374 \\ 49 & 6 & 43 & 49 & 7 & 42 & 45100.730 & -0.120 & 0.10 & -1.1 & -168.561 & 0.618 \\ 49 & 7 & 42 & 49 & 8 & 41 & 56491.550 & -0.227 & 0.20 & -1.0 & 882.951 & 2.683 \\ 50 & 5 & 45 & 50 & 6 & 44 & 60330.520 & -0.079 & 0.10 & -1.0 & -1198.218 & -0.879 \\ 50 & 6 & 44 & 50 & 7 & 43 & 46205.050 & 0.076 & 0.10 & 0.7 & -368.393 & 0.343\end{array}$

rms deviation: 1.1837

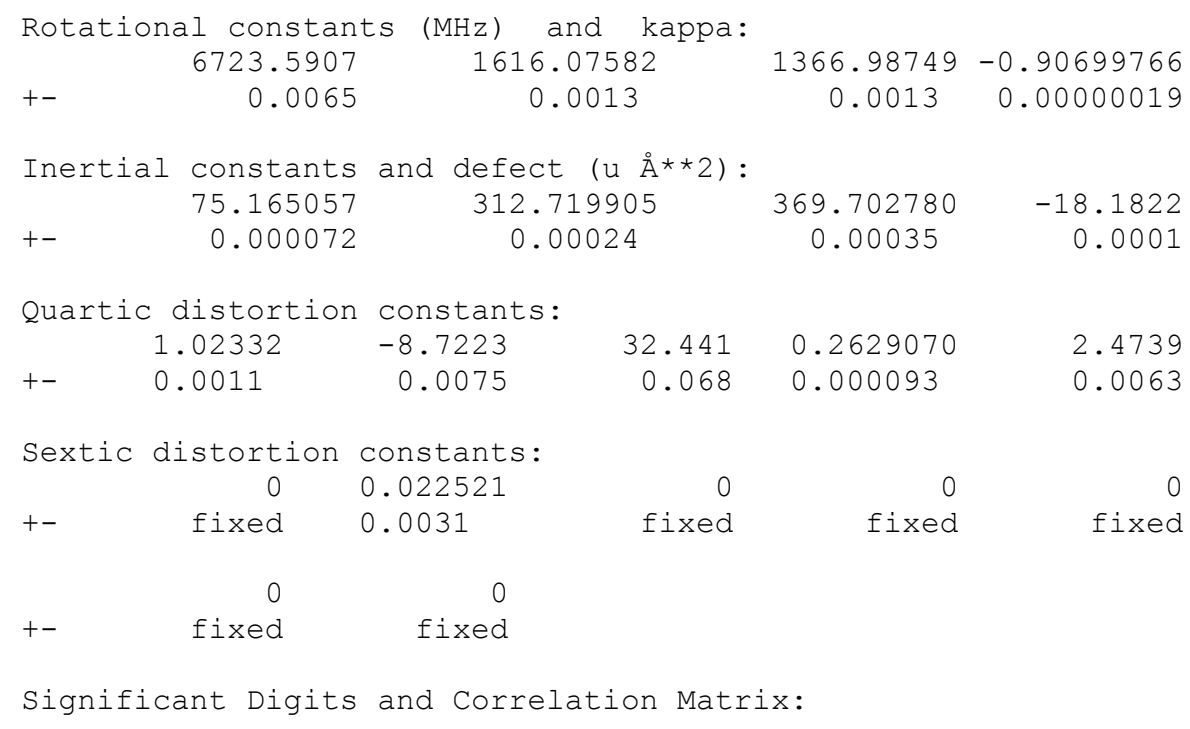

8

$\begin{array}{rrrrrrrr}0.400 & 0.423 & 0.264 & 0.624 & 0.596 & -0.116 & 0.047 & 0.594 \\ 9 & 0.944 & 0.911 & 0.393 & -0.069 & 0.076 & -0.017 & 0.339 \\ & 9 & 0.920 & 0.426 & -0.030 & -0.218 & 0.158 & 0.422 \\ & & 6 & 0.159 & 0.011 & -0.104 & 0.103 & 0.187 \\ & & & 5 & -0.121 & -0.156 & 0.138 & 0.921 \\ & & & 5 & -0.109 & -0.017 & 0.047 \\ & & & & 7 & -0.832 & -0.355 \\ & & & & & 5 & 0.303\end{array}$

